
BW. $07226 \in 120,00$

Sunigar 



\section{ALGAE JAPONICAE}

MUSEI BOTANICI LUGDUNO-BATAVI,

$4 \operatorname{terog\pi }$

W. F. R. SURTNGAR,

Meth Yage Phil Nat De, in Tuir. I. B. Prof Ond.

(BDIOIT SOCIBTAS SCTENTIARM HOLTANDICA QTAB FARTMIT MST.

HARTEVT.

TYPIS HBREDUM LOOSJBS.

1870. 
SOCIETATI SCIENTIARUM HOLLANDICAE HARLEMI OBLATUM D. 26 M. MARTII 1868. 


\section{A L G A E.}

Algas japonicas hic descriptas collegerunt v. clar. DE SrEBoLD, nec non v. doct. Buerger et nob. Textor et Bisschop. Loci natales fere nusquam indicati erant sed plerasque species e viciniis $\mathrm{N}$ a $\mathrm{ng}$ a s a $\mathrm{k}$ insulae $\mathrm{Ki}$ u s u provenisse conjicere licet.

\section{A TOM ACEAE.}

Observatio de mensuris.

Mensurae Algarum ab auctoribus diversis modis exprimuntur, ab aliis millimetri, ab aliis lineae parisiensis ("') $)$ ab aliis denique pollicis plerumque anglici partibus, iisque numeribus fractis sive vulgaribus sive decimalibus. Equidem, mensuras a metro sumtas prae pollicum et linearum varietate praeferendas ducens, hoc loco ut jam alibi, omnes mensuras expressi partibus millemetri millesimis, quas micromillemetros $(m . m . m$.$) vocavit \mathrm{cl}$. HARTing, et quas brevitatis causa singula littera $\mu$ designavi. Addidi tamen, quo facilius cum aliorum auctorum indiciis mea comparari possent, lineae parisiensis partes, micromillemetrorum numero respondentes, et in Diatomaceis nonnullis easdem pollicis anglici, quem vero, ne cum aliis pollicibus confunderetur, non signo omnibus communi (") sed littera $i$ distinxi.

Striae in Diatomacearum cellulis quantum inter sese distent exprimi solet striarum in spatio certo contentarum numero. Sed alii striarum numerum in centesima parte lineae parisiensis, alii eundem in millesima parte pollicis anglici contentum indicant. Spatium quod hic selectum est, ut in eo numerus striarum definiretur, $25 \mu$, facilem cum utroque illo indicationis modo comparationem sinit. Vix enim differt ab hoc spatio millesima pollicis anglici pars; $0,001 i$ exacte respondet $25 \frac{1}{2} \mu$. Itaque si striae 50 in spatio $25 \mu$ observantur, adsunt 51 in $0,001 i$. Discrimen ipsius ob- 
servationis errore probabili superatur. Lineae parisiensis pars centesima respondet $22 \frac{1}{2} \mu$. Itaque a numero striarum in $25 \mu$ observatorum decima pars detrahenda est ut ad numerum in $\frac{1}{100}{ }^{\prime \prime \prime}$ reducatur; $50 \mathrm{v}$. c. striis in $25 \mu$ respondent 45 in $\frac{1}{100}{ }^{\prime \prime \prime}$ et ita porro.

\section{CYCLOTELLA KG.}

CYCLOTELLA MENEGHINIANA KG.

Kützıng, Die Kieselschaligen Bacillariën, pag. 50, Tab. XXX, fig. 68; Species Algarum, pag. 19.

Inter Algas aquae dulcis Japoniae. - Tab. I, fig. 1.

- CYClotella Sinensis Ehr. var.? C. Annulata Suringar.

Index praecursorius Algarum Japonicarum Mus. Bot. L. B. (Annales Musei Bot. Lugd. Bat. 1867, T. III, p. 256.) No.2.

Diametro duplo majore $\left(70 \mu=\frac{1}{32}^{\prime \prime \prime}\right)$ spatio intermedio intramarginali depresso latiore inaequaliter concentrice lineolato, latere connexivo lineari angusto. - Tab. I, fig. 2.

Inter Algas aquae dulcis Japoniae, semel.

Obs. Distinctionem inter Cyclotellae (Discopleae) species Ehrenbergianas sinensem, atmosphericam, atlanticam et oregonicam aliquanto dubiam esse jam monuit doct. RaLFs in Pritchard, a listory of Infusoria ed. IV, 1861, pag. 812. Quantum e figuris in opere Ehrenberghiano Microgeologia colligi potest conjungendae videntur :

1. D. sinensis a EHr. (Microgeologie, Tab. XXXIV, VII, 2) cum D. sinensi? (Microgeologie, Tab. XXXIX, II, 10) ob utriusque marginem angustiorem et strias approximatiores quam in ceteris.

2. D. sinensis (Microgeologie, Tab. XXXIX, I, fig. 16) cum ejusdem varietate $\gamma$. stellata Eнк. (Microgeologie, Tab. VI, II, fig. 3) propter costulas marginales aliquanto longiores et praesertim multo remotiores.

3. D. sinensis $\beta$ (D. atmospherica?) EHR. (Microgeologie, Tab. XXXIV, VII, fig. 3), striis longioribus disci minuti diametrum superantibus granulatis insignis, cum D. at- 
mospherica (Microgeologie, Tab. XXXIX, I, tig. 17); a quibus denique D. atlantica (Microgeologie, Tab. XXXIX, II, fig. 29) et D. oregonica (Microgeologie, Tab. XXXVII, II, fig. 3) vix differre videntur.

E tribus hisce speciebus vel varietatibus cum prima comparanda est nostra $C$. annulata. Distinguitur diametro duplo majore, sulco latiore lineolato. Unicum tantum vidi specimen, in eoque costulas nonnullas in granulorum series solutas. An orta per copulationem e Cyclotella sinensi $\alpha$ ?

\section{ARACHNODISCUS DEANE.}

\section{ARACHNODISCUS ORNATUS EHR.}

Ehrenberg, in Actis Academiae Berolinensis 1849. A. japonicus Shadbolt, Transactions of the microscopical Society of London, IV, Nov. 1849. Pritchard, A history of Infusoria, ed. IV, Tab. XV, fig. 18-21. (non Sмгтн, A Synopsis of the British Diatomaceae, I, Tab. XXXI, fig. 256).

Ad Algas marinas Japoniae. - Tab. I, fig. 3.

Obs. Videntur primi detexisse hujus generis specimina doct. Toppings et alii in guano ex Ichaboe (in Africa), annis 1843 - 1844. Brevi post cl. Ehrenberg, de speciminibus e guano Patagonico sumtis descripsit summ Hemiptychum ornatum, quod tamen nomen, scilicet nomen generis, postea abolitum est propter ejus consonantiam cum insecti cujusdam nomine Hemiptychue. Nomen genericum nunc receptum proposuit exp. DeAne, in Societate microscopica Londinensi (anno 1847) verba faciens de eadem hac pulcherrima Diatomacea, quam in Algis japonicis, ut opinabatur primus, invenerat, et cui nomen Arachnodisci japonici dederat; describere vero genus et speciem, et descriptionem publici juris facere, omisit (Qurterly Journal of Microscopical science, VI, pag. 188); quo factum est ut qui brevi post illam descripsit eodem A. japonici nomine, not. SнарвоLт, (Transactions of the Microscopical Society of London, Tom. IV, 1849, Nov. 14) generis et speciei auctor putaretur, usque dum etiam hujus viri in nomine specifico auctoritas anteriori EHRENBergianae locum cessit. Mox alteram speciem, A. Ehrenbergii, distinxit Phycologus americanus BAILEY, de speciminibus e California (Puget sound) allatis, quam speciem recepit et illustravit el. Smrн (a Synopsis of the British Diatomaceae, I, pag. 26, Tab. XXXI, fig. 256) nisus auctoritate cli. DE BRÉBısson et iconis photographicae testimonio. 
Attamen idem SMirt primus confusionis auctor fuit, synonymon huic speciei adjiciens nomen $A$. japonici SнADbolt. Quod in epistola vituperat cl. BaILEY (Quarterly Journal of Microscopical Science, VI, pag. 162, 1858) genus detectori Deane tribuendum esse declarans, sed sibi speciem novam $A$. Ehrenbergii, ab A. japonico diversissimam, vindicans.

Additis a clo. Ehrenberg $A$. indico et $A$. nicobarico (Microgeologie, Tab. XXXVI, fig. 35) genus Arachnodisci revisioni subjecit cl. WALKER ArNot' (Quarterly Journal of Microscopical Science, VI, 1858, pag. 159, 195). Duas servavit species, ceteras recte ad synonymiam relegans.

Servavit autem :

1. A. Ehrenbergii BaILex, ad quam A. indicum Eнr, et

2. A. ornatum Енг., ad quam $A$. japonicum $\mathrm{S}$ н Арвоцт et $A$. nicobaricum Eнr. retulit. Nova denique descripta species A. Grevilleanus Hardmann (Transactions of the Microscopical Society of London, VIII 1865, pag. 47, Tab. V) A. Ehrenbergii valde affinis, tantum centro costulis stellato distinctus, eodem jure ac A. indicus Eнr. cum A. Ehrenbergï conjungenda mihi videtur.

Huic vero quem dixi auctori, $\mathrm{cl}^{\circ}$. W A t.Ker Annott, $A$. Ehrenbergii (qualis in opere Sмiтhiano laudato illustratur) quoad patriam est ,vera species japonica”; alteram contra, a $\mathrm{cl}^{\circ}$. Shadbolt japonicam vocatam, revera de speciminibus Africanis ab eo descriptam esse opinatur. Suspicatur porro, $\mathrm{cl}^{\mathrm{m}}$. BaIley nomen suum $A$. Ehrenbergii primum quidem speciei ,revera japonicae” dedisse, postea tamen idem hoc nomen applicavisse ad illum A. japonicum Sнadbolt quem ab origine Africanum credit.

Duplex haec erroris suspicio, et vel in ipso Phycologo qui primus utramque speciem distinxit, mihi minus probabilis est. Neque videntur argumenta a $\mathrm{cl}^{\circ}$. WALKer Arnotr allata alind probare, nisi quod ipse fortasse utramque speciem e California acceperit. Fieri etiam possit ut $A$. ille Ehrenbergï BAILEx una cum $A$. ornato Eнr. in mari japonico crescat. Nostra vero specimina japonica indubie ad $A$. ornatum Eнr. pertinent, i. e. ad eandem illam speciem quam SHADвоцт japonicam vocavit, hujusque igitur auctoris designationem loci natalis confirmant.

\section{LIPAROGYRA Eнr.}

LIPAROGYRA CIRCULARIS Enr. emend.

Cellulis solitariis cylindricis $17 \mu-24 \mu\left(\frac{1}{13} 0^{\prime \prime \prime}-\frac{1}{94}{ }^{\prime \prime \prime}\right)$ crassis, $40-74 \mu\left(\frac{1}{5}^{\prime \prime \prime}-\frac{1}{3}^{1}{ }^{\prime \prime \prime}\right)$ longis, costis internis circularibus $6-13,5 \mu-7 \mu\left(\frac{1}{450}{ }^{\prime \prime \prime}-\frac{1}{3 \frac{1}{3}}{ }^{\prime \prime}\right)$ inter sese distantibus. 
Syn. L. circularis EHr. et L. scalaris EnR. Nicrogeologie, Tab. XXXIV.

In caespite Cladophorae tomentosae in statione humida sylvestri (ad truncos arborum?) Japoniae. Index praecurs. 1. c. $\mathrm{N}^{\circ} .4$. - Tab. I, fig. 4, $a-g$.

Obs. Speciminum parcorum quae observare licuit mensurae hae erant. :

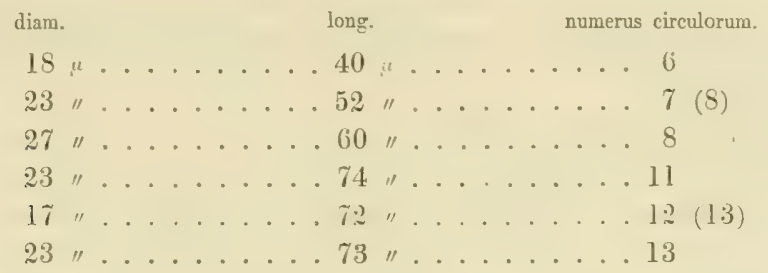

Circuli in omnibus aequo intervallo $5-7 \mu$ plerumque $6 \mu$ distantes, constant costis validis introrsum acutis, internae faciei parietis cellulae affixis, eamque, in sectione optica transversa, denticulorum serie, intermediorum validiorum utrinque sensim minorum, ornantibus. Ter vidi circulos vicinos bifurcatione aut ramulo inter se conjunctos (fig. 4, c, d,e). Quamquam post multos irritos conatus tandem cellulam in apicem vetere et ejusmodi faciem orbicularem observare milhi contigerit, tamen nimis obscura hujus faciei imago erat quam ut aliquid novi observationibus jam notis addere potuerim (fig. $4, g$ ). Tres apiculos qui huic faciei adscribuntur me conspicere putavi quidem, haud vero hoc modo clarius quam in cellulis a latere visis apparebaut. Margo coronatus erat reliquiis membranae quae cellulas binas ante divisionem probabiliter includit; nulli denticuli aderant; neque circulus pororum vel depressionum quales in genere Porocyclia describuntur aderant. Distinctionem inter hoc genus et Liparogyram ceteroquin obscuram esse jam monuit RALrs in Pritchardr opere de Infusoriis laudato.

Liparogyra circularis EHr. descripta sine figura, et $L$. scalaris EHr. illustrata (Microgeologie, 'Iab. XXXIV) sine descriptione, inter se non alio modo differre videntur quam numero circulorum; hune vero numerum in nostris speciminibus vidimus a longitudine cellularum pendere et cum incremento cellulae a 6 ad 13 accrescere, qui numerus probabiliter per cellulae divisionem ad dimidiam partem denuo reducitur. Itaque $L$. scalaris EnR. non nova species habenda videtur serl eadem ae L.circularis. 


\section{NITZSCHIA Hassald.}

\section{NITZSCHIA COMMUNIS RAB.}

$\mathrm{R}_{\mathrm{ABEnhons}}$, Die Algen Sachsens, resp. Mittel-Europa's No.949, Flora Europaea Algarum aquae dulcis et submarinae, I, pag. 159. Synedra notata KG, Bacill. Tab. III, fig. 33 .

Inter Algas aquae dulcis Japoniae. - T'ab. I, fig. 5, $a-c$.

\section{NITZSCHIA SIGMOIDEA (Nitzsch) Sm.}

Sмгтн, Brit. Diat. I, pag. 38, Tab. XIII, fig. 104; Sigmatella Nitzschii Kg. Spec. Alg. pag. 18.

Inter Algas aquae dulcis Japoniae. - Tab. I, fig. 6.

$$
\text { NITZSCHIA LINEARIS (Ag.) Sм. }
$$

Sмгтн, Brit. Diat. I, pag. 39, Tab. XIII, fig. 110 ; Tab. XXI, fig. 110.

Inter Algas aquae dulcis Japoniae. - Tab. I, fig. 7, $a-d$.

\section{NITZSCIIA AMPHIOXYS (Енв.) Sм.}

Sмiтн, Brit. Diat., I, pag. 40, Tab. XIII, fig. 105; Eunotia amphioxys Ehr. Verbr. Am.; KG. Bacill., pag. 36, Tab. XIX, fig. 44, XXX, fig. 1.

Inter Algas aquae dulcis Japoniae. - Tab. I, fig. 8, $a-e$.

Obs. Forma vulgaris, utrinque subito contracta apiculis productis obtusis, qualis in figuris Kützingianis (Die Kieselschaligen Bacillariën, Tab. XXIX, fig. 44, Tab. XXX, fig. I) et in speciminibus a doct. RABENHorst editis (Bacill., n ${ }^{0}$. 19) apparet. Minus bene convenit cum figura Ehrenbergrana (Microgeologie, Tab. XXXIV, VIII.) ejusdem speciei e Japonia.

$30 \mu-38 \mu(-44 \mu)=7^{1} 5^{\prime \prime \prime}-5^{1} 9^{\prime \prime \prime}\left(-\frac{1}{5}^{\prime \prime \prime}\right)$ longa, punctis carinalibus validis $12-15$ in $25 \mu$. 
NITZSCHIA CUNEATA Suriggar. 1. c.

Linearis, vel rarius medio leviter attenuata, apicibus subito cuneatis $50 \mu=\frac{1}{4} 0^{\prime \prime \prime}$ longa, nodulis $16-15$, striis saepissime obscuris 35 in $25 \mu$, pro majori parte ipsis valvis (11 $\mu-12 \mu=\frac{1}{210}{ }^{\prime \prime \prime}-\frac{1}{190}^{\prime \prime \prime}$ latis) latera connexiva latitudine aliquanto superantibus incumbens. - Tab. I, fig. 9, $a-d$ (et $9^{*}, a-l$, de speciminibus Hollandicis).

Syn.: Surirella multifasciata $\mathrm{KG}_{\mathrm{G}}$. (pro parte?)

Inter Algas aquae dulcis Japoniae.

Obs. Sectio cellulae transversa ut in omnibus Nitzscliiis ${ }^{1}$ ) rhomboidea. Quum obliqua jacet, angulo cum serie nodulorum (carina auctorum) superne versa, cellula praebet formam in brevioribus lanceolatam, in longioribus linearem utrinque sensim angustatam, apicibus in nonnullis productis obtusis. Rarius tamen hoc modo conspicitur; pleraque specimina ipsis valvis incumbunt.

Haec species, novo nunc nomine proposita, ipsa non incognita erat; est enim eadem quae nomine Surirellae multifasciatae $\mathrm{K}$ G. quum in Prodromo Florae Batavae tum in $\mathrm{Ob}$ servationibus meis Plycologicis in Floram Batavam inter indigenas Hollandiae commemoratur. At nomen hoc evanuit, nempe, condito genere Nitzschiae et varïs speciebus Synedrae, Surirellae et Eunotiae in illud receptis, S. multifasciata KG. a v. cl. Sumtu inter synonymas suae Nitzschiae linearis (Frustuliae linearis AG.) relegata est. Quam quidem speciei Sumpianae vel Agardhinae cum Kützingiana congruentiam ex toto negare non auserim, deficientibus nihi speciminibus ab auctoribus ipsis definitis ${ }^{2}$ ). Id tantum hoc loco moneam, specimina nostra (fig. $9^{*}, a-l$ ) antea ad Surirellam multifasciatam $\mathrm{K}_{\mathrm{G}}$. collata et cum hujus speciei descriptione et figura in opere KützıN-

1) (Налтzsch, Hednigia 1859, $\mathbf{n}^{\circ}$.4), Schumaxx, Prenssische Diatomeën, Schififten der Königlich Pysicalisch-Oekonoinischen Gesellschaft zu Königsberg, III, 1862, pag. 186.

2) Cl. Sмrtr (1. c.) se specimina authentica Frustuliae linearis AG., a. v. cl. Grerille accepta, cum spccie sua comparasse monet. S. multifasciatam KG. de speciminibus Gallicis notam habuit. Quae autem ipse vidi specimina Gallica, hoc nomine signata, pleraque (e. gr. illa in collectione DessrazTk̀rES, ed. II, $\mathbf{n}^{0}$. 209 cum icone valde mediocri) ad $N$. linearen SмIтн quidem pertinent (alia vidi ad $N$. tenuem Surti d. rabustam RAв. 1. c. pag. 159 necnon ad $N$. dubiam Smitн referenda); nulla vero cum figura et descriptione KüTzıN grasis laudatis satis conveniunt. Ipse KüтziNgrus specimina authentica speciei suae promulgavit in Algarum. decadibus suis $\mathrm{n}^{\circ}$. 32 . At in exemplari, ad consulendum mihi parato, species in testula deficiebat. Quamquam igitur, de descriptione et figura laudatis, virisimillimum mihi videtur, S. multifasciatam KG. propriam speciem generis Nitzschiae, a N. lineari Sмrтн diversam, constituere et specimina nostra ad speciem illam KütziNGIANגм pertinere, tamen, re non penitus certa, et quo vitaretur omnis confusio, novum nomen praeferendum duxi prae illo Nitzschiae multifasciatae ( $\mathrm{KG}_{\mathrm{.}}$ ) quod alioquin legibus nomenclaturae convenisset. 
Grano: Die Kieselschaligen Bacillariën, pag. 60, Tab. III, fig. 47 converientia, ad Nitzschiae genus quidem referendas esse, sed discrepare a speciei $N$. linearis typo, qualis quum in descriptione et figuris Sminiasis tum in collectionibus venalibus apparet. Differunt autem non tantum cellulis brevioribus, verum etiam valvarum forma utrinque abruptius cuneata et earundem latitudine, latus connexivum superante, quo fit ut, dum in $N$. linearis grege omnia fere individua latera haec oculis offerant, nostrae contra maior pars ipsis valvis incumbat. Accedit quod in $N$. lineari striae transversae omnino desunt.

Una quidem e varietatibus, hoc tempore $N$. lineari adscriptis, quoad formam cellularum nostrae valde similis est, nempe ea quam doct. RABenHorst in Algarum decadibus suis sub $\mathrm{n}^{\circ}$. 482 nomine Synedrae seriantis Br£B. promulgavit et in opere suo c. t. Flora Europaea Algarum aquae dulcis et submarinae I, pag. 158, ut varietatem $d$ seriantem, $N$. lineari adjunxit. Mensuris ac forma nostram aequat; striae tantum nullae illic indicantur, et cellulae per series inter se cohaerent. At dubito quin etiam haec cum $N$. lineari recta conjungatur. De nomine porro BRÉbissoniano quod doct. Rabenhorst illi apposuit, alligata synonyma Frustulia seriante BRÉB. in coll. Desmazières $n^{\circ} .907$ et editionis alterius $n^{0} .207$ passim notandum: hoc de editione prina fortasse valere, haud vero de altera quam solam consulere potui. Haec enim sub numero laudato et nomine Frustuliae seriantis non habet Nitzschiam, sed venustissimam illam Naviculam seriantem (Bве́в.) KG. Bacill., pag. 92, quain Ds. Rabenhorst 1. c. Tom. I, pag. 172 memorat.

In - Nitzschia Stagnorum $\mathrm{R}_{\mathrm{AB}}$, quam ipse auctor nuper cum $N$. thermali $\mathrm{K}_{\mathrm{G}}$. conjunxit, praevalent formae medio attenuatae, quae in wostra rariores sunt; striis insuper multo densioribus illa distinguitur.

NITZSCHIA PALEA (Kg.) Sr.

Sмтти, Brit. Diat., II, pag. 89; Grünow, Die Oesterreichischen Diatomaceën, Abh. d. K. K. Zoöl. Bot. Ges. in Wien, XII, pag. 561, Tab. XII, fig. 3, 7. Synedra palea KG. Bacill. pag. 63, Tab. III, fig. 27, IV, fig. 2.

Inter Algas aquae dulcis Japoniae. - Tab. I, fig. 10.

NITZSCHIA TENUIS SM. var. C. PARVA Rab.

Rabenhonst, Flora Europaen Algarum aquae dulcis et submarinae, pag. 158, Alg. Dec. $\mathrm{n}^{\circ}$.943, Hedwigia, II, 'Tab. IV, fig. 6.

Inter Algas aquae dulcis Japoniae. - 'Tab. I, fig. 11. 
NITZSCIIIA FLEXELLA SURingar, 1. c.

Facie carinali levissime sigmoidea $42-47 \mu=\frac{1}{5}^{\prime \prime \prime}-\frac{4^{\prime}}{4}{ }^{\prime \prime \prime}$ longa, a medio $5 \mu=$ ${ }_{4}^{4} \frac{1}{5}{ }^{\prime \prime}$ lato sensim angustata, apicibus obtuse truncatis, valvis linearibus propter apices oblique abscissos parallelogrammatis angusti formam referentibus, nodulis $18-22$ in $25 \mu_{\text {。 }}$ - Tab. 1, fig. 12.

Inter Algas aquae dulcis Japoniae.

Obs. Intermedia inter $N$. flexam Schumann, Prenssische Diatomëen, in Schr. d. Kön. Plyys. Oek. Ges. zu Königsberg, III, 1862, pag. 186, Tab. VIII, fig. 23. et $N$. curvulae (EnR.) Sмiтn formam parvam Grünow in $\mathrm{R}_{\mathrm{AB}}$. Dec. no. 1712.

\section{NITZSCHIA ARCULUS Suringar. 1. c.}

Leviter arcuata exacte linearis apicibus obtusis, nodulis 15 in $25 \mu ; 66 \mu=\frac{1}{3}^{\prime \prime \prime}$ longa, $4 \mu=\frac{1}{5+}^{\prime \prime \prime}$ lata. - Tab. I, fig. 13 .

An forte varietas parva arcuata $N$. vermicularis (KG.) Hantzsch?

Inter Algas aquae dulcis Japoniae.

Obs. Eandem hanc formam immixtam vidi speciminibus Europaeis $N$. tenuis $\boldsymbol{\beta}$ mediae $\mathrm{H}_{\mathrm{antzsch}}$ in $\mathrm{R} \mathrm{AB}$. Dec. $\mathrm{n}^{0} .945$.

\section{MAVICULA BORY.}

\section{NAVICULA APPENDICULATA (Ag.) KG.}

Kützisg Bacill. pag. 93, Tab. III, fig. 28, IV, fig. 1; Grünow, Uéber neue oder ungenügend gekannte Algen, Abh. d. Kön. Kais. Zoöl. Bot. Ges. in Wien X, pag. 553, Tab. II, fig. $29 b, d$.

Inter Algas aquae dulcis Japoniae. -- Tab. I, fig. $14 a-d$.

Obs. $18-22 \frac{1}{2} \mu=\frac{1}{12} \frac{1}{6}^{\prime \prime \prime}-\frac{1}{100^{\prime \prime \prime}}=0,0007-0,0009$ i longa; $4-6 \mu=$

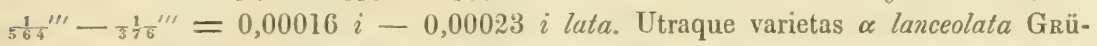
Now (fig. $a, b$ ) et $\beta$ exilis Grünow (fig. $c . d$.) 
NAVICULA CUSPIDATA KG.

Kützing, Bacill., pag. 94, 'Tab. III, fig. 24. Sмптн, Brit. Diat. I, pag. 47, Tab. XVI, fig: 131.

Inter Algas aquae dulcis Japoniae. - Tab. I, fig. 16, $a-c$.

Obs. $80 \mu=\frac{1}{2} \overline{3}^{\prime \prime \prime}=0,00315$ i longa; $19 \mu-222_{2}^{1} \mu=\frac{1}{119^{\prime \prime \prime}}-\frac{1}{10} 0^{\prime \prime \prime}=0,00075$ $i-0,0009 i$ lata, striis transversis $33-37$ in $25 \mu$.

Ejusdem: forma longicollis Suringar 1. c.

Apicibus longius protractis levissime capitatis. - Tab. I, fig. 16, $d, e$.

Cum praecedente.

Obs. Mensurae et striarum intervalli ut in praecedente.

\section{NAVICULA PRODUCTA Sм.?}

Sмiтн, Brit. Diat., I, pag. 51, tab. 'Tab. XVII, fig. 144. Grünow, 1. c. pag. 543 , Tab. II, fig. 35.

Inter Algas aquae dulcis Japoniae. - Tab. I, fig. 15.

Obs. Determinatio dubia quia strus non vidi; $54 \mu=\frac{1}{4}^{\prime \prime \prime}=0,0021$ i longa, $14 \mu=\frac{1}{160} 0^{\prime \prime \prime}=0,00055$ i lata.

\section{NAVICULA AFFINIS EIr.}

(Eurenberg, Verbr. Am.) Kützing, Bacill., pag. 95, Tab. XXVIII, fig. 65;

Grünow, 1. c. pag. 543, Tab. III, fig. 2.

Inter Algas aquae dulcis Japoniae. - Tab. I, fig. 17.

Obs. $63 \mu=\frac{1}{3}^{\prime \prime \prime}=0,00245$ i longa, $17{ }^{\prime \prime}={ }_{3 \frac{1}{3} 3^{\prime \prime \prime}}=0,00067$ i lata. Strias non vidi.

Ejusdem? FORMA MINOR.

$32 \mu={ }_{77^{\prime \prime}}{ }^{\prime \prime \prime}=0,00126$ i longa, $12 \mu={ }_{1 \frac{1}{8} 8^{\prime \prime \prime}}=0,0047$ i lata.

Inter Algas aquae dulcis Japoniae. - Tab. I, fig. 17*. 
NAVICULA FIRMA KG, a Major Grinow.

Grünow l. c. pag. 543, Tab. III, fig. 1; Nav. lata (microstoma) KG. Bacill., Tab. III, fig. 51 .

Inter Algas aquae dulcis Japoniae. - Tab. II, fig. 18, $l, m$.

Obs. Striae longitudinales fere 25 in $25 \mu$, transversales tenuissimae 50 in $25 \mu$; $70 \mu-82 \mu=\frac{1}{32}^{\prime \prime \prime}-\frac{1}{2} 7^{\prime \prime \prime}=0,0027 i-0,00321 i$ longa, $18 \mu-22 \mu=\frac{1}{1}^{\frac{1}{2}{ }^{\prime \prime \prime}}$ $-{ }_{1^{1} 3^{\prime \prime \prime}}=0,0007 i-0,00086$ i lata.

\section{Ejusdem: forma triundulata Suringar 1. c.}

Lateribus connexivis undulato-inflexis, hinc valvis praeter margines rectos linea triunduluta notatis. - Tab. II, fig. 18, $a-k$.

Cum praecedente.

Obs. Forma hujus speciei est analoga illi quam doct. GrüNow (1. c.) in N. limosa descripsit et ad quam N. truncatam KG. (Bacill., Tab. III, fig. 34) retulit. Striae ut in praecedente. $82-108 \mu=\frac{1}{2} 7^{\prime \prime \prime}-\frac{{ }^{\prime}{ }^{\prime \prime \prime}}{2}=0,0032 i-0,0042 i$ longa, utroque latere $22 \mu-23 \mu=\frac{1}{1}{ }^{\prime \prime \prime}-\frac{1}{93^{\prime \prime \prime}}=0,00086 i-0,0009 i$ lata. Undulationes laterum in speciminibus siccis satis opacis aegre conspiciuntur. Aderant vero in speciminibus fig. $a-d$. siccis delineatis similiter ac in fig. $e-k$ de speciminibus aquae immersis. Porro notandum, in hac specie cellulas propter valvarum formam convexam saepe obliquas jacere.

\section{PINNULARIA EHR.}

PINNULARIA MAIOR (KG.) SмIтн.

Sмiтн, Brit. Diat., I, pag. 54, Tab. XVIII, fig. 162. Navicula major KG.

Bacill., pag. 97.

Inter Algas aquae dulcis Japoniae. - Tab. I, fig. 19. 
PINNULARIA VIRIDIS (Nitzsch) SMTr.

Sмгтн, Brit. Diat., I, pag. 54, Tab. XVIII, fig. 163. Navicula viridis (Niтzsci) Kg. Bacill., pag. 97.

Inter Algas aquae dulcis Japoniae. - Tab. II, fig. 20.

\section{PLEUROSIGMA Smith.}

\section{PLEUROSIGMA SPENCERII Smith.?}

Sмгтн, Brit. Diat., I, pag. 68, Tab. XXII, fig. 218.

Inter Algas aquae dulcis Japoniae. - T'ab. II, fig. 21.

Obs. $100 \mu=\frac{1}{2} s^{\prime \prime \prime}=0,0038 i$ longa, $13 \mu={ }_{1 \frac{1}{7} 5^{\prime \prime \prime}}=0,0005 i$ lata. Strias non vidi, hinc determinatio aliquanto dubia. Color stramineo-fuscus.

\section{SCOLIOPLEURA Griirow.}

GrüNow, Ueber neue oder ungenügend gekannte Algen, 1. c. pag. 554.

$$
\text { SCOLIOPLEURA ORNATA (KG. ?) SURINGar l. c. }
$$

Valvis elliptico-rhomboideis, longitudine $\left(125 \mu=\frac{1^{\prime \prime}}{1^{\prime}}\right)$ latitudinem quinquies superantibus, valde convexis, costa mediana leviter utrinque arcuata, striis obliquis cruciatis validis 25 in $25 \mu$ facile in granulorum series duplo pauciores solutis, lateribus connexivis late linearibus obtusis truncatis, membranula connexiva ipsa paullum et curvata et obliqua. - Tab. II, fig. 22, $a-f$.

Syn. ? Surirella? ornata KG. Bacill., pag. 62, Tab. III, fig. 54.

Inter Algas marinas Japoniae.

Obs. Unicum vidi specimen quod initio, obliquum jacens, faciem fig. $c$ delineatam ostendebat; deinde illud alio modo arte verti ut valvas $(a)$ et latus connexivum (b) animadverterem. Valvae valde convexae sunt et carinatae quidem, quo fit ut 
sectio transversalis fere sexangularis sit et cellula in eo positu quo valvam suam ostendat difficulter retineatur. Eadem fortasse est haec species atque illa quam cl. KützINg, in opere suo laudato Tab. III, fig. 54 icone illustravit et pag. 62 descripsit, nomine Surirellae ornatae sed dubitans de genere, quod dubium etiam auctoribus posterioribus remansit. Si eadem est ac nostra, cl. Kürzıng specimen illud Genuense obliquum jacens delineavit, ut nostra in fig. $c$ repraesentatur.

Granulorum series in figura nostra $d$ a sculptore aliquanto spatiosius dispositae sunt. Ceterum numero duplo minori hae apparent quam striae in speciminibus minns amplificatis numerantur, propterea quod confiniis serierum ipsarum et intervallorum iis aequalium striae illae hic respondent.

\section{STAURONEIS EHR.}

\section{STAURONEIS FENESTRA EnR.}

(Ehrenberg, Verbr. Am.) Kützing, Bacill, pag. 105, 'Tab. IX, fig. 6.

Inter Algas aquae dulcis Japoniae. - Tab. II, fig. 23.

Obs. $16 \mu=\frac{1}{14} \sigma^{\prime \prime \prime}=0,00063 i$ longa.

\section{CYMbella Ag.}

CYMBELLA MACULATA KG.

Kützing, Bacill., pag. 79, Tab. IV, fig. $2 a, 2 b$.

Inter Algas aquae dulcis Japoniae. - Tab. 1I, fig. 24.

Obs. Specimina ad $55 \mu=\frac{1}{4}{ }^{\prime \prime \prime}=0,0022 i$ longa; striae 24 in $25 \mu$.

\section{CYMBELLA VENTRICOSA AG.}

(Agardh, Compectus criticus Diat.) Kützing, Bacill., pag. 80, Tab. VI, fig. 16. Rabenhorst, Die Sïssiv. Diat. pag. 23, Tab. VII, fig. 9.

Inter Algas aquae dulcis Japoniae. - Tab. II, fig. 25.

Obs. Specimina ad $22 \frac{1}{2} \mu=\frac{1}{10} \bar{\sigma}^{\prime \prime \prime}=0,00088 i$ longa; alia fere duplo minora. 


\section{AMPHIPLEURA Ka.}

\section{AMPHIPLEURA JAPONICA SURINGar l. c.}

Leviter sigmoidea a medio utrinque sensim attenuata apicibus obtusis, triplicata pellucida, subtilissime transverse striata, striis circa 60 in $25 \mu ; 85 \mu-105 \mu=$ $3_{2}^{1} \gamma^{\prime \prime \prime}-\frac{1}{2} 2^{\prime \prime \prime}$ longa, medio $14 \mu=\frac{1}{16} 0^{\prime \prime \prime}$ lata. - Tab. 1I, fig. 26.

Inter Algas aquae dulcis Japoniae.

Obs. A. pellucidae $\mathrm{K}_{\mathrm{G}}$. forma et mensuris proxima, at flexura diversa. A. rigida KG. firmior et pro ratione angustior est.

\section{TETRACYCLUS RaLfs.}

TETRACYCLUS LACUSTRIS RALFS.

(RALFs in Annals and Magazine of natural history, vol. XII) Kürzing, Bacill., pag. 127, Tab. XXIX, fig. 70. Raвenhonst, Flora Eur. Alg. I, pag. 302.

Inter Algas aquae dulcis Japoniae. - Tab. II, fig. 27.

Obs. Ex uno tantum latere observata, hinc determinatio aliquanto dubia.

\section{COCCONEIS EUR.}

COCCONEIS CONSOCIATA KG.

Kützing, Bacill., pag. 72, Tab. V, fig. 8, VIII, fig. 6.

Ad Algas marinas Japonicas. - Tab. II, fig. 28. $\left(\frac{980}{1}\right.$, non $\left.\frac{49 g}{1}\right)$.

Obs. Late elliptica $14 \mu-18 \mu=\frac{1}{16} \sigma^{\prime \prime \prime}-\frac{1}{2} \sigma^{\prime \prime \prime}$ louga, limbo nullo, granulorum seriebus utrinque $13(-15)$, spatio medio lineari. 


\section{GOMPHONEMA AG.}

\section{GOMPHONEMA CAPITATUM EHR.}

Ehrenberg, Infus., pag. 2]7, 'Tab. XIII, fig.'2. Kützing, Bacill., pag. 86,

Tab. XVI, fig. 2. Sмгтн, Brit. Diat., I, pag. 80, Tab. XXVIII, fig. 237.

Inter Algas aquae dulcis Japoniae. - Tab. II, fig. 29.

\section{RHOICOSPHENIA Griinow.}

RIOICOSPHENIA CURVATA (KG.) GrüNow.

GrüNow, Ueber neue oder ungenügend gekannte Algen, 1. c. pag. 511, de genere. Gomphonema curvatum KG. Bacill., pag. 85, Tab. VIII, fig. 1-3; Sмттн, Brit. Diat., I, pag. 81, Tab. XXIX, fig. 245.

Ad Algas aquae dulcis Japoniae. - Tab. II, fig. 30.

Obs. Latus valvare aliquanto latius quam in iconibus laudatis. Striae 21 in $25 \mu$.

\section{DESMID I A E A E.}

\section{CLOSTERIUI Nitzsci.}

\section{CLOSTERIUM JAPONICUM SURINGar 1. c.}

Anguste fusiformis latitudine 18 - 20 longior levissime arcuata pallide viridula, striis longitudinalibus tenuissimis circa 23 in $25 \mu$, suturis transversis $3-\mathbf{5}$; $0,4-0,6 m m .=\frac{1^{\prime \prime \prime}}{6}-\frac{1^{\prime \prime \prime \prime}}{4}$ longa. - 'Tab. II, fig. 31 .

Inter Algas aquae dulcis Japoniae.

Obs. Closterio intermedio RALFs proxima, at major et striis fere duplo densioribus diversa. 


\section{ZYGNEMACEAE.}

\section{SPIR0GYRA LK.}

\section{SPIROGYRA LINEATA SURINgar 1. c.}

Caespite atro-viridi, cellulis (sterilibus) cylindraceis, diametro $\left(70 \mu=\frac{1}{3}{ }^{\prime \prime \prime}\right) 1 \frac{1}{2}-3$ plo longioribus dense longitudinaliter lineolatis, fasciis chlorophyllophoris tribus parallelis subrectis. - Tab. IV.

In aqua dulci Japoniae, coll. Siebord \& Textor.

Obs. De insignibus hujus speciei indole ac evolutione fusius disserui in concione Academiae Regiae Disciplinarum Nederlandicae ad diem $26 \mathrm{~m}$. Octobris anni superioris (Verslagen en Mededeelingen der Koninklijke Academie van Wetenschappen, T. II, ser. 2, cum tabula valde mediocri; Archives Néerlandaises des sciences exactes et naturelles, publ. par la société Hollandaise des sciences à Harlem, T. III, pag. 116.) Cellulae juniores in apice trichomatis habent fascias chlorophyllophoras spiraeformes, numero plerumque 5, anfractibus $1-2$ laxiusculis (Tab. nostra IV, fig. $\left.3 ; a, a^{\prime}, a^{\prime \prime}\right)$. Quae spirae mox per trichomatis incrementum in fascias subrectas mutantur et ejusmodi conspiciuntur in cellulis aliquanto ab apice renotis (fig. $3, b$ ). Brevi post, chlorophyllo evanescente, fasciae solvuntur in fibras decolores vel sordide flavescentes quae, cellularum parietem internam dense vestientes, ean lineolis illis longitudinalibus speciei peculiaribus ornant (fig. $3, c$ ). Novae interim ortae vesicularum chlorophyllarum series, initio rectae (fig. $3, d, e$ ) deinde ramis sertiformibus auctae, hoc pacto novas spiras fingunt, primariis haud dissimiles quamquam origine diversae (fig. $3, f, g$ ), quae posthac ipsae denuo in fascias tres latiores subrectas convertuntur (fig. $h, i, i^{\prime}$ ). Sic demum oritur status adultus, fasciis hisce chlorophyllophoris tribus latioribus subrectis et simul lineis illis longitudinalibus, e chlorophylli vetusti solutione ortis, notatus et in partibus trichomatis ab ipso apice juniori remotis conspicuus.

Affinis est species nostra Spirogyrae orthospirae NAEGELI, in qua pari modo fasciae chlorophyllophorae in cellulis terminalibus spiraeformes inchoari et deinde rectae fieri videntur. Quod autem in vivis accuratius indagandum. Specimina enim sicca hujus speciei quum in aqua einolliuntur multo minus bene formam suam recuperant quam nostrae Spirogyrae lineatae. Multo firmior haec est. Porro distinguitur ab illa praeterquam notis microscopiis etiam colore caespitis olivaceo-nigrescente. 


\section{OSCILLARIN AE.}

\section{SIROSIPHON KG.}

SIROSIPHON VERMICULARIS Suringar. 1. c.

Repens, parce et vage ramosus, ramis patentibus aut rectangularibus elongatis cum trichomate flexuosis, cellulis 1 - 2-, in apicibus clavato-tumidis ramorum 2 - pluri-seriatis aerugineis, tubo gelineo crasso, trichomatis primarii lamelloso fuscescente, ramorum hyalino cinctis, diametro $\left(7,6 \mu-15 \mu=\frac{1}{2} \frac{1}{9}{ }^{\prime \prime \prime}-\frac{1}{15 \pi^{\prime \prime \prime}}\right.$ s. $v$. $13 \mu-25 \mu=\frac{1}{175}^{\prime \prime \prime}-\frac{1}{90}{ }^{\prime \prime \prime}$ c. v.) brevioribus. - Tab. III, A.

Inter Cladophoram tomentosam SuR., in terra humida Japoniae.

Obs. Proxime accedit ad formas quas cum S. ocellato (DILL.) KG. conjunxit Doct. Rabenhorst (Flora Eur. Alg. aq. d et subm. II, pag. 2S6) S. intermedii KG. et Braunii KG. (Spec. Alg. pag. 317, Tab. Phyc., II, Tab. XXXVI), ab illa autem distincta ramis remotioribus vagis elongatis, vaginis pallidioribus, trichomatibus flaccidioribus, cellulis non granulatis, ab hac parietibus gelineis crassioribus, ab utraque cellulis in apicibus clavato-incrassatis ramorum 2-pluri-seriatis.

\section{HYPHEOTHRIX KG.}

\section{HYPIEOTHRIX ANGUINA SuRINGar. 1. c.}

Trichomatibus pallide chalybeo-aerugineis tortuosis, cellulis minute granulatis, diametro ( $\left.5 \mu-6 \mu=\frac{1}{4} \sigma^{\prime \prime \prime}-\frac{1}{3} \sigma^{\prime \prime \prime}\right) \frac{2}{3}$. brevioribus, vaginis inconspicuis, apiculis paullum attenuatis simpliciter oblusis. - Tab. III, B.

Parasitica in Spirogyra lineata SuR. Japoniae.

Obs. Inseratur juxta $H$. involventem (Arescr.), KG. Species Alg., pag. 269 (Scytonema myochrous AREsch., Alg. Scant. exsicc. $\mathrm{n}^{\circ} .48$ ), quae facile distinguitur trichomatibus suis conglutinatis, intra vaginam distinctam saepe interruptis. 


\title{
CONFERVACEAE.
}

\section{CHAETOMORPHA KG.}

\author{
CHAETOMORPHA MaCrotona Suringar l. e.
}

Pallide viridis flaccida crispata, cellulis cylindricis diametro $(137-150 \mu=$ $\left.\frac{1}{16}^{\prime \prime \prime}-\frac{1}{15}{ }^{\prime \prime \prime}\right) 2-5$ plo longioribus parietibus fibroso-striatis. - Tab. VI, A.

In mari Japonico, coll. T'extor.

\section{CLADOPIORA IKG.}

CLADOPHORA INSIGNIS (Ag.) Kg. $\gamma$ Fuviatilis Kg.

Kützing, Spec. Alg. pag. 406, Tab. phyc. Vol. IV, Tab. XXXVIII.

In aqua dulci (fluente?) Japoniae.

Obs. Obscure viridis simplex caespitosa palmaris, trichomatibus radicula ramosa affixis rigidiusculis cellulis infimis decoloribus longissinis et diametrum septies superantibus, superioribus diametro $\left(60 \mu=96 \mu=\frac{1}{3}^{\prime \prime \prime}-\frac{1}{2} 7^{\prime \prime \prime}\right) 1 \frac{1}{2}-3$ plo longioribus, parietibus crassis rigidis. Obsita erat filamentis variis Confervae vel Oedogonii cujusdam primordiis.

\section{CLADOPIJORA TOMENTOSA SURINGAR 1. e.}

Caespite sordide et pallide viridi tomentoso-intricato expanso humilissimo, trichomatibus basi scutelliformi affinis flaccidis crispis sub-simplicibus, ramis ramulisque parcis conformibus patentibus vel angulo recto $e$ mediis cellulis egredientibus elongatis varie curvatis, cellulis diametro versus apices sensim decrescente $\left(27 \mu=11_{2}^{1}=\right.$ $\left.\frac{1^{\prime \prime}}{4^{\prime \prime}}-\frac{1}{20} \sigma^{\prime \prime \prime}\right) 2-3$ plo longioribus, contentis pallidis tenuissimis. - Tab. V.

In statione humida sylvestri (ad truncos arborum?) Japoniae.

Obs. Stationem humidam sylvestrem indicant prothallia Flicum, rami Hepaticarum aliaque immixta. Aderant in caespite Liparogyra circularis Eur., Sirosiphon vermicularis SuR. et Bulbotrichia botryoides $\mathrm{KG}_{\mathrm{G}}$. Caespes tam intricatus, ut hoc respectu ad Aegagropitae conditionem fere accedat. Trichomata singula magnam similutidem habent figurae, qua Kützrngrus (T'ab. Pliyc., Vol. IV, 'lab. LIV) Cladophoram subsimplicem ( $\mathrm{Cl}$. simpliciusculam Hooker et HARVEY) a se (Spec. Alg., pag. 412) descriptam illustravit. At haec duplo crassior. Etiam magis a nostra diversam haec species se ostendit, si figuram 1. c. laudatam in Cryptogamia antarctica auct. Hooker et Harvey respicias. 


\section{ULVACEAE.}

PHYCOSERIS KG.

PIIYCOSERIS AUSTRALIS KG.

Kützing, Phyc. gener., pag. 298; Spec. Alg., pag. 477; Tab. Phyc., Vol. IV, 'Tab. XXIV.

In mari Japonico. - Tab. VI, B.

Obs E basi brevissimâ oblique cuneatâ valde dilatata transverse oblonga, margine lacerata, passiin pertusa, colore coerulescente viridi, phyllomata $38_{\mu}=\frac{1}{60^{\prime \prime \prime}}$ crasso, cellulis $5-6$ gonis sine ordine dispositis, diametro $16 \mu-20 \mu=\frac{1}{14}^{\prime \prime \prime}-\frac{1}{110^{\prime \prime \prime}}$ (duplo majori quam in Plyycoseri gigantea) purietibus firmis crassiusculis. Phycoseris smaragdina KG. ad cujus formam latifoliam specimen nostrum Japonicum primum retuleram (Index praec, , 1. c. $\mathrm{n}^{0} .39$ ), distinguitur cellularum parietibus tenuioribus. Phycoseris lobata KG. a Phycoseri australi KG. vix differre mihi videtur.

\section{ENTEROMORPHA LINK.}

\section{ENTEROMORPIIA INTESTINALIS LINK.}

(Link, Hor. Plyye. Berol.) Kg. Spec. Alg., pag. 478; Tab. Plyc., Vol. VI, Tab. XXXI.

In mari Japonico, Textor.

ENTEROMORPHA COMPLANATA KG.

Kutzixg, Pliyc. germ., pag. 248; Species Alg., pag. 480; Tab. Plyyc., Vol. VI, Tiab. IXXIX.

In mari Japonico; Textor et Siebold.

\section{CHROÖLEPIDEAE,}

BULBOTIRICHIA KG.

BULBOTRICHIA BOTRYOIDES KG.

Kützing, Tab. Plyycol., Vol. IV, pag. 22, Tab. XCVII.

Inter caespitem Cladophorae tomentosae Sor., in statione humida sylvestri Japoniae. 


\title{
COELOBLASTEAE.
}

\section{CODIUI Ag.}

\author{
CODIUM ELONGATUM AG.
}

Agardi, Spec. Alg. I, pag. 454; Kg. Spec. Alg., pag. 501 ; Tab. Phyc., Vol. VI, Tab. XCVI.

In mari Japonico.

Obs. Forma linearis angusta (angustior quam figura Kützingiana in Tabulis pliycologicis laudata) versus apices et etiam alibi inaequaliter dilatata, remote (plerumque bis) furcata, ad $40 \mathrm{~cm}$. longa. Cellulae periphericae clavatae magnae (aliquanto majores etiam quam in aliis ejusdem speciei individuis illas observavi) $1,3 \mathrm{~mm} .-2 \mathrm{~mm} .=5^{\prime \prime \prime}-\frac{10^{\prime \prime \prime}}{11}$ longae, apicibus $0,35 \mathrm{~mm} \cdot-0,45 \mathrm{~mm} \cdot=\frac{2{ }^{\prime \prime \prime}}{13}-5^{\prime \prime \prime}$ latae, parietibus tenuibus. Dissepimenta, ubi adsunt, tenuia.

Omnia nostra specimina sicca decoloria, fuscescenti-cana.

CODIUM LATUM Suringar 1. c.

Fronde cuneala plana bifurcata medio latissima, laciniis ex axillo rotundato divergentibus sursum paullum angustatis apice obtusis sive bilobis, rhizomio obconico concavo affixa; cellulis periphericis oblongo-clavatis $0,38 \mu=\frac{1^{\prime \prime \prime}}{6}$ longis, $70 \mu=\frac{1}{3}{ }^{\prime \prime \prime}$ crassis, parietibus tenuibus; cellulis tubulosis in strato frondis interno prope periphericarum originem dissepimentis crassissimis praeditis. - 'Tab. VII.

In mari Japonico.

Obs. Frons $24 \mathrm{~cm}$. longa, medio $3 \frac{1}{2} \mathrm{~cm}$., in ipsa bifurcatione $9 \mathrm{~cm}$. lata. Laciniae ad basin 4 et $3 \mathrm{~cm}$. latae. Color atro-viridis.

Dissepimenta illa peculiaria crassissima medio canali exiguo pertusa vel potius propter parietis internae plicam circularem, divisionem cellulae efficientem, in centro haud concretam incompleta, quae in cellulis tubulosis frondis internae juxta cellularum periphericarum originem animadvertuntur, eadem ac in hac nostra specie observavi in Codio Mulleri KG. (Tab. plyyc. VI, pag. 34) et in Codio Lindenbergï (BINDER hb.) KG. (eodem loco). Species nostra juxta $C$. Lindenbergii Binder et $C$. damaecorne (BoRx) $\mathrm{K}_{\mathrm{G}}$. (eodem loco) inserenda est, nisi potius fortasse cum utraque in unam speciem conjungenda. 


\section{aCANTHOCOdIU Surivgar 1. c.}

Habitu et structura Codii at cellulis periphericis spinescenti-acuminatis.

ACANTHOCODIUM Fragile Suringar 1. c.

Fronde cylindrica $2 \mathrm{~mm}$. crassa subdichotome ramosa fragilissima, cellulis periphericis oblongo-clavatis acuminatis magnis, $0,57 \mathrm{~mm}$. $=\frac{1^{\prime \prime \prime}}{4}$ longis, $0,076-0,115 \mathrm{~mm}$. $=\frac{{ }^{\prime \prime \prime}}{30}-\frac{1}{20}{ }^{\prime \prime \prime}$ latis, parietibus in apice spinescenti-acuminato valde incrassatis, inferne tenuissimis. Fructus non aderant. - Tab. VIII.

In mari Japonico, Textor.

Obs. Cellulae periphericae in sectione frondis quasi longe pedicellatae apparent, inferiori scilicet parte anguste lineari, apice obovato-dilatato. Quod tamen ejusmodi explicandum est, ut cellulae in inferiore parte, ubi paries tenuissima est collabantur, haud vero in apice duro et firmo. Idem hoc de Codii speciebus valet; in hisce cellulae pari modo formam veluti pedicellatam ostendunt si integram frondis sectionem inspicias, per totam contra longitudinem dilatatae naturali sua forma apparent, si singulas cellulas e superficie evellas et ejusmodi sejunctas microscopio subjicias (vide Tab. VII in Codio lato, fig. 3 et 5.) Hoc vero in nostro Acantlocodio propter eximiam cellularum fragilitatem difficilliuum. Si singulas cellulas e fronde solvere tentes, fere semper abrumpuntur apices spinaeformes. Non nisi e praeparatione diu in chloreti calcici solutione asservata et emollita tandem mihi contigit paucas cellulas integras solvere, quales in figuris $4 a-c$ delinatae sunt. Tota frons fragilissima. Fragmenta tantum pauca aderant eaque parva et manca e quibus de integrae frondis mensura nil conjicere licet. Colorem cernebant cinereo-viridem.

\section{PHAEOSPOREAE.}

\section{HYDROCLATHRUS BonY.}

\section{HYDROCLATIIRUS CANCELLATUS BORY.}

Rony, in: Dictionnaire classique d'histoire naturelle, Vol. VIII, pag. 419; Kützing, Tab. Pliycol., Vol. IX, pag. 24, Tab. LII. II; Encoelium clathratum Ag. Spec. Alg., Vol. I, pag. 412. Kg. Spec. Alg., pag. 552.

In mari Japonico, SIEBold. 


\title{
PHYLLITIS KG.
}

\author{
PIIYLLITIS DEBILIS (AG.) KG.
}

Kützing, Spec. Alg., pag. 567; Laminaria debilis Ag., Spec. Alg., I, pag. 120.

In mari Japonico, TexтoR. - Tab. IX, B.

Obs. Proxime accedit ad formam externam Punctariae debilis a KG. (Tab. Phyc. vol. VI, Tab. XLVII; Phycolapathi debilis a KG. Spec. Alg. pag. 483.) sed structura est generis Phyllitidis $\mathrm{KG}$. Refert fortasse veram L. caespitosam J. AG. (Nov. Fl. Suec., Species, genera et ordines Fucoidearum, pag. 130) a v. cl. KüтZING, propter synonymiam allatam $L$. cuneateae Sunr., ad genus suum Plyycolapathum relatam. At in Phyllitide haud minus quam in Punctaria debili KG. videntur formae frondis e stipite sensim dilatatae ad alias abruptius dilatatas sensim transire neque certis limitibus ab hisce separari. Quam ob rem etiam nostram ad $P h$. debilem (AG.) referendam esse putavi.

\section{ALARIA Grét.}

ALARIA PINNATIFIIIA HaRveY.

Hanver, Characters of new Alyae, chiefly from Japan and adjacent regions collected by Charles Wright in the North Pacific exploring expedition, under Captain JoHn Rodgers (Proceedings of the American Academy of arts and sciences, vol. IV, pag. 327, Oct. 1859).

„Stipite ancipite sursum complanato crispato-marginato in costam latam apice desinente, lamina latissima profunde pinnatifida, laciniis oblongis simplicibus furcatisve sinubus rotundatis, pinnis crassis obovatis brevibus". HARver l. c.

In mari Japonico, Siebolı. - '́ab. X (forma junior).

Obs. Specimina nostra juniora parva sterilia, stipite tenui ancipite.

\section{LAMINARIA LAMOUR.}

\section{LAMINARIA JAPONICA ARESGHOUgit.}

Areschough (Plyceae capenses, pag. 29) Phyceae novae et minus cognitae in maribus extra-europaeis collectae (Act. Reg. Soc. Scient. Upsal., Ser. III, vol. I, pag. 362). 
„Stipite compresso in laminam basi longe attenuatam lineari-lanceolatam integram fascia fusca latiore percursam mox evanescente" Aresch. 1. c.

Syn. Fucus saccharinus 'I'nuмв. Flora Japonica, pags. 346, teste v. cl. Aresch. de specimine in Herb. Thunв. - Laminaria saccharoides Indicis praecursorii nostri l. c. $n^{\circ} \cdot 49$.

In mari Japonico, v. Siebold. - Tab. XI et XII.

Obs. Radix fibrosa. Stipes firmus breviusculus ( $8 \mathrm{~cm}$. longus) compressus superne cuneato-complanatus sensim transiens in laminam giganteam lineari-lanceolatam apice longe productam (in nostris $2 \frac{1}{3}-3 \frac{1}{2}$ metros longam, $12-25 \mathrm{~cm}$. latam) nonnusquam bifido-pertusam, in medio fascia crassiore lata diffusa percursam, in margine et apice tenuiorem undulatam. Fascia haec peculiaris, basin frondis totam occupans, mox ad tertiam ejus latitudinis partem restricta, nusquam certis limitibus circumscribitur, sed versus margines tenuiores sensim diffluit et ante apicem frondis tota evanescit. Subinde dividitur et per certum spatium costas duas, rarius tres, valde vagas et diffusas simulat. Pari modo ipsa frons, sive inferue, sive in media vel superiore parte nonnunquam bifido-pertusa conspicitur. Superficies frondis in basi et superne in fascia longitudinaliter et reticulatim rugulosa; margo cum apice frondis tenuior laevis undulatus.

Habet structuram Laminariae bifidae Post. et Rupr., sed cellulas duplo minores; neque magis quam haec species vera Laminaria esse milhi videtur. Fructus ignoti.

\section{FUC ACEAE.}

\section{SARGASSUII (Ag. e. p) IKG. \\ SARGASSUM PILULIFERUM (TuRNer) Ag.}

Agardh, Spec. Alg. I, pag. 27; J. G. Agardh, Species genera et ordines Fucoidearum, pag. 289; Kützing, Spec. Alg. pag. 616; Fucus pilulifer Turner, Historia Fucorum, vol. I, pag. 146, Tab. LXV.

\section{Forma CAPILLARIs.}

Foliis superioribus capillaribus enerviis.

In mari Japonico. 


\title{
SPONGOCARPUS IK.
}

\author{
SPONGOCARPUS HORNERI (TURNer) KG.
}

Kützing, Spec. Alg., pag. 631. Tab. Plyycol., vol. X, Tab. LXXXIX. Fucus Horneri Turner, Hist. Fuc., vol. I, pag. 34, Tab. XVII; Sargassum Horneri AG., Spec. Alg., I, pag 38; J. G. Agardi, Spec. gen. et ord. Fucoid., pag. 290.

In mari Japonico, Siebold et Textor.

Obs. Ex omni parte cum descriptionibus et iconibus laudatis convenit.

$$
\text { SPONGOCARPUS SISYUIBRIOIDES (TunNer) KG. }
$$

Küтzing, Spec. Alg., pag. 632; Tab. Plyycol., vol. X, Tab. XCI; Fucus sisymbrioides Turner, Hist. Fuc., vol. II, pag. 150, Tab. CXXIX; Cystophyllum sisymbrioides J. G. Agardh, Spec. gen. et ord. Fucoid., pag. 234.

In rupibus submersis freti van der Capellen ad littus insulae Nippon prope Simonoseki, Bisschop.

Obs. Fragmentum terminale determinationis haud indubiae.

$$
\text { SPONGOGARPUS IIEMIPIYLLUS (TuRNE) Kg. }
$$

Kützing, Spec. Alg., pag. 632; Tab. Plycol., vol. X, Tab. XC; Fucus hemiphyllus Turner, Hist. Fuc., vol. III, pag. 86, Tab. CLXIX; Sargassum hemiphyllum Agardh, Spec. Alg., I, pag. 39; J. G. Agardh, Spec. gen. et ord. Fucoid., pag. 331 .

In mari Japonico, Siebold et Textor.

Obs. Accurate convenit cum descriptione et figura Turnerianis laudatis.

\section{HALOCHLOA IKG.}

IIALOCHLOA PATENS (Ag.) KG.

Kürziva, Phycologia generalis, pag. 368; Spec. Alg., pag. 632; Tab. Pliycol., vol. XI, Tab. I; Sargassum patens Agardi, Spec. Alg., I, pag. 27; J. G. Agardu, Sp. gen. et ord. Fucoid., pag. 228; Fucus pilulifer $\beta$ maior Turner, Hist. Fuc., vol I, pag. 46.

In mari Japonico, Sifbold.

Obs. Determinatio aliquanto dubia propter lacinias e basi tenuiore versus bifurcationes dilatatas. Vesiculae coronatae. 
HALOCHLOA SERRATIFOLIA (AG.) KG.

Kutzing, Spec. Alg., pag. 633; Tab. Plyycol., vol. X, Tab. XCIX. (Fucus serratifolius Agardh, Dec. 31) Sargassum serratifolium J. G. Agardh, Spec. gen. et ord. Fucoid., pag. 291; Fucus longifolius var. tenuifolius Torner, Hist. Fuc., vol. II, pag. 88 , Tab. CIV.

In littore sinus Sima Bara prope oppidulum Tsa Faja insulae Kiusiu, Bisschop; TeXtor; Bürger.

\section{HALOCHLOA SILIQUASTRUM (TunNer) KG.}

Küтzing, Spec. Alg., pag. 634, Tab. Phycol., vol. X, Tab. XCVII. Fucus siliquastrum Turner, Hist. Fuc., vol. II, pag. 26; Tab. LXXXII; Sargassum siliquastrum AGardH, Spec. Alg., I, pag. 21; J. G. Agardh, Spec. gen. et ord. Fucoid, pag. 292.

In littore sinus Simu Bara propre vicum Sagami insulae Kinsin, Bisscrop.

Obs. Fragmenta terminalia. Determinatio haud indubia.

\section{HALOCILLOA SCOPARIA (TURNER) KG.}

Kützing, Spec. Alg. pag. 634, Tab. plyycol. vol. X; Tab. XCIII; Fucus scopa. rius Turner, Hist. Fuc., vol. II, pag. 156, Tab. CXXXII; Sargassum scoparium Agardh, Spec. Alg., I, pag. 26; J.G. Agardi, Spec. gen. et ord. Fucoid., pag. 292.

Forma aERocystis MinoRibus.

In mari Japonico, TEXтоR.

\section{MYAGROPSIS KG.}

\section{MYAGROPSIS THUNBERGII (MERTENS) KG.}

Kützing, Spec. Alg., pag. 635; Tab. Phycol., vol. X, Tab. XCIII, Fucus Thunbergii Mertens in: Roth, Catalecta Botanica, vol. IlI, pag. 104, Tab. III, fig. $a-d$; Turner, Hist. Fuc., vol. II, pag. 158, Tab. CXXXIII; Cystophyllum Thunbergii J. G. Agarde, Spec. gen. et ord. Fucoid., pag. 233.

In saxis submersis littoris sinus Sima Bara prope Fimi, et valli urbis Nangasaki, insulae Kiиsiu, Bisschop, Bürger. 


\section{DICTYOTEAE.}

SPATHOGLOSSUM KG.

SPATHOGLOSSUIJ HULTIPARTITUM (SurR) Kg.

Kützing, Spec. Alg. pag. 560, Tab. Plyycol., pag. 21, 'Tab. L, Vol. IX.; Zonaria multipartita SonR.

In mari Japonico. - Tab. IX, A.

\section{FLORIDEAE.}

\section{HORMOCERAS KG.}

HORHOCERAS FLACCIDUM HARVEX.

Kützing, Tabulae phycologicae, XII, pag. 21, Tab. LXIX.

,Minutulum delicutulum gelatinosum, forcipibus temissimis clausis, zonis inferioribus parum elevatis, articulis inferioribus diametro $6-7$ plo, mediis 3 plo longioribus, superioribus aequalibus, interstitiis maxime hyalinis". HARver l. c.

In mari Japonico, Gloeopeltidi intricatae Sur. affixa. - 'Tab. XIII.

Obs. $2 \frac{1}{2} \mathrm{~cm}$. alta ; diameter trichomatis primarii $75 \mu=\frac{1}{30}{ }^{\prime \prime \prime}$.

\section{CAMPYLAEPHORA J. AG.}

CAIIPYLAEPIIORA IIYPNAEOIDES J. AG.

J. G. Agardh, Species genera et ortines Algarum, II, pag. 149.

In mari Japonico, Siebold. - Tab. XIV.

\section{PORPHYRA Ag.}

PORPIIYRA VULGARIS AG.

(Agardi, Bot. Zeitung, 1837.) Kutzing, Spec. Alg., pag. 699. . In mari Japonico, Техток. 


\section{SCHIZYMENIA J.AG.}

JAC. G. Agardi, Species genera et ordines Algarum, II, pag. 169.

SCHIZYMENIA? LIGULATA Suringar l. c.

Frondibus membranaceo-carnosis $2-4$ ex apice stipitis brevis filiformis prodeuntibus longe ligulatis paullulum undulatis et curvatis, simplicibus aut supra medium subdichotomis, e margine passim ramulosis, apicibus denuo angustatis (plerumque abruptis).

Color coccineo-fuscus. Frondes ad $22 \mathrm{~cm}$. longae, ad $2 \mathrm{~cm}$. latae, siccae membranacene, madefactae carnosae. - Tab. XV.

In mari Japonico, Textur.

Obs. Fructibus deficientibus genus incertum. Forma externa cum Grateloupiis nonnullis convenit, structura potius Schizymenice videtur.

\section{CHONDIUS Lamour.}

\section{CHONDRUS PUNCTATUS SURIngar 1. c.}

Fronde purpurea membranacea madefacta carnosa molli flabellata inde a basi repetite (-4) dichotoma segmentis cuneatis superne dilatatis, apicibus obtusis sive bilobis passim latissimis proliferis, cystocarpiis in inferiore frondis parte sitis mumersissimis minimis punctiformibus uni paginae proxius in frondem immersis, paullum prominentibus. - Tab. XVI.

In mari Japonico, 'Texтok.

Habitu cum specimine parvo Chondri crispi convenit; colore et structura molliori perenchymatica Iridaeas fere attingit.

\section{GIGARTINA Lamour.}

GIGARTINA TENELLA HARvey.

Harver, Characters of neiv Algae..... collected by Chardes Wright..... cet. (Proceedings of the American Acad. of arts and sciences, IV, pag. 527). 
„Fronde pusilla compressa anguste lineari distiche ramosa, ramis altemis sparsisve arcuatis attenuatis, ramulis paucis subulatis patentibus saepius secundis apicibus acutis." HARVEY 1. c.

In mari Japonico, 'Textor. - Tab. XVII, A.

Obs. Specimina Hakverand non vidi; nostra sterilia, $4-6 \mathrm{~cm}$. alta a medio fere ramosa circa $1 \mathrm{~mm}$. crassa; sicca cartilaginea, madefacta gelatinosa; aqua dulci mox dissolvuntur.

\section{GIGARTINA INTERMEDIA SURINGAR 1. c.}

Fronde compresso-filiformi inde a basi subdichotoma ramis patentibus flexuosis bipinnatis apicibus ramulisque lanceolato-dilatatis complanatis sulcatis.

Color purpurascens; specimen siccum $6 \mathrm{~cm}$. altum, $1-1 \frac{1}{2} \mathrm{~mm}$. crassum. Tab. XVII, B.

In mari Japonico, T'extor.

Obs. A praecedente facile distinguitur fronde magis compressa apicibus dilatatis sulcatis, tela laxiori et cellulis corticalibus brevioribus. Ceteroquin habitu inter $G$. acicularem Lamour et $G$. falcatam (Hering) J. AG. I. c. II, pag. 266, intermedia. Fructus non aderant.

\section{GLOEOPELTIS J. AG.}

JAC. G. Agande, Algae maris Mediterranei et Adriatici, pag. 68; Species genera et ordines Algarum, II, pag. 234.

I. GLOEOPELTIS genuina.

Rami secundarii ex axe primario longe adscendentes, intra spatium pericentrale frondis dichotomi et ramosi.

GLOEOPELTIS TENAX (TurNer) J.Ag.

J. G. Agardh 1. c., Fucus tenax Turner (Ann. of Bot., II, pag. 367.) Historia Fucorum II, pag. 141, Tab. CXXV.

In mari Japonico, SiebordD.

Obs. Specimina manca, sed de structura insigni cognoscenda.

II. COELOPELTIS.

Rami secundarii ex axe primario patentissimi brevissimi, intra spatium pericentrale frondis non (vel vix) ramosi. 


\section{GLOEOPELTIS CAPILLARIS.}

Fronde pusilla capillari terete vel leviter compressa anguste tubulosa, praster siphonem centralem hujusque ramos primarios brevissimos patentissimos, tubum extermu sustentantibus, vacua, 2-4-furcata, ramis patentibus sive ex axillo rotundato adscendentibus apice attenuatis, cystocarpiis in ramis exsertis, mecleo in stratum periphericum toto immerso, tetrachocarpiis cruciatis in cortice sparsis. - Tab. XVIII.

Diameter $\frac{1}{2}$ mm. non attingit.

Ad rupes maritimas, Mlitadiri; r. Siebold.

Obs. Incolis cibo est, quod apparuit e schedula a collectore Japonensi, nomine Kékai, speciminibus, quae v. cl. Siebot,D obtulerat, adjecta: „Crescit”, belgico sermone scripsit ,Mitadiri ad rupes maritimas et colligitur digitis, quibus ne agglutinetur prius cinere adspergitur. Parandi modus: aqua emolliatur et infundatur aceto cum jure. Etiam ad pultem praeparandum adhibetur."

Specimina adeo erant in charta conglutinata et implexa, ut vel pauca ex informi mole integra extrahere difficillimum esset. Attamen labor non ingratus fuit. Non tantum enim contigit, ut forma frondis externa satis bene cognosceretur et structurae anatomicae explorandae facultas obtineretur, verum etian fructus utriusque generis aderant, ita ut nullum de speciei indole dubium relinqueretur.

Structura in plerisque convenit cum illa quam cl. J. G. AgardH in G. tenace observavit et in opere laudato primus accurate descripsit. Tantummodo aliquanto simplicius nostra species constructa est. Frondem anguste tubulosam percurrit sipho articulatus, e serie simplici cellularum cylindricarum, endochromate colorato et parietibus crassis praeditarum, conformatus. Certis locis emittit ramos sibi conformes, at brevissimos patentissimos, plerumque alternos subinde tamen oppositos, tubum externum frondis sustentantes, vel potius ipsos, ramis novis sibi exortis et solide concretis, frondem hanc tubulosam exstruentes. Cellularum parietes hoe loco plerique in gelatinau amorpham confluentes rarius limites suos externos distinctos ostendunt. Tela fit quae dicitur epenchymatica, sed e contentorum coloratorum (gonidiorum) seriebus facile ramorum directio animadvertitur ('Tab. XVIII, fig. 5, 7). Primum ubi interiorem parten strati peripherici frondis conficiunt, hujus superficiei internae paralleli conspiciuntur, variis quidem directionibus e ramis primariis decurrentes, alios in frondem adscendentes, alios descendentes, alios denique obliquos vel transversos, sed omnes communiter spatium vacuum internum ambientes. Deinde versus peripheriaw ipsam froudis ramulos suos creberrimos quum laterales tum termiuales eosque iterato dichotomos emittunt, quibus denique frondis stratum corticale constituitur.

Ut paucis dicam, tota structura anatomica ita explicari potest, ut ex axe 
centrali, ipso simplice serie cellularum constituto, rami exeant brevissimi patentissimi iterata di-polychotomia late penecillatim ramosi, qui denique penecilli in frondem continuam tubulosam solide concrescant.

Haud minus clare quam in sectionibus transversis et longitudinalibus haec structura apparet in frondis fragmento aqua dulci partim soluto et levissima pressione distento. Quod in genere affini Endotrichia ('lab. XXII, fig. 2) figura illustravi.

Versus basin frondis tubi spatium angustatur, donec omnino evanescat (T'ab. XVIII, fig. 4) et axis centralis stratum periphericum tangat (fig. 2); semel (an fortasse in fragmento alieno immixto?) hoc loco axem primarium ramis secundariis secum adscendentibus cinctum vidi (fig. 3). Quod contra in Gloeopeltide tenace consuetum. In hac antem specie omnes rami ex axe centrali longe adscendentes per spatium frondis pericentrale decurrunt et jam antequam in penecillos illos stratum periphericum frondis constituentes desinant, ramificantur, ramisque spatium illud pericentrale densius vel laxius farciunt.

De fructu cystocarpico ejusque placenta centrali pulcherrime dendroidea jam fusius tractavit Cl. J. G. Agardh 1. c. ; illustrata est in 'Tab. nostra XVIII, fig. $8 a-a^{\prime \prime \prime}$. Nucleus ex axis ramo ortum ducens totus immersus est in stratum periphericum; fructus e fronde exsertus. Tetrachocarpia, ut in ceteris, cruciata in strato corticali sparsa.

Speciem novam hoc loco propositam antea (in Indice praecursorio, 1. c. No. 67) ad G. furcatam (Post. et Rupr.) J. AG. I. c. II, pag. 235 (Dumontiam furcatam Postrus et Ruprecht, Illustrationes Algamm Oceani Pacifici, imprimis septentrionalis, auctoribus A. Postels et F. Ruprecht, pag. 19, 'T'ab. XL, fig. 79) retuleram; sed visis speciminibus hujus speciei authenticis quum in Herbario Kewensi tum a v. cl. J. G. Agardir benevole mecum communicatis, a nostra illam alienam esse et potius ad typum $G$. coliformis HARVEx accedere apparuit. Frons pusilla paucos millimetros alta e basi capillari nox distenditur et in tubum pro ratione amplum inflatur. Quae vidi specimina erant simplicia aut furcata ad $\frac{1}{2} \mathrm{~cm}$. alta, superne ad amplitudinem fere $1 \mathrm{~mm}$. inflata. Fructus aberant, structura eadem erat ac in Gl. coliformi. Dubito an pro specie propria habenda sit vel an non potius primordia tantum $G$. coliformis vel $G$. intricatae (vide supra) referat.

\section{GLOEOPELTIS COLIFORMIS HaRVEY.}

Harvey, Characters of new Algae..... collected by Charles Wright..... cet. (Proceedings of the American Acad. of arts and Scirnces, 1V, pag. 327).

„Fronde intestinaeformi nembranacea, filo centrali mox evanido, cava et inflata subsimplici, basi et apice attenuata, mun apice prolifera, mnc furcata; cystocarpiis mumerosissimis" HARVEY I. c. 
In mari Japonico, Stebold. - 'Tab. XIX (de speciminibus in Herb. Kewensi).

Obs. Specimina pauca eaque mancissima in herbario aderant Gl. tenaci immixta; speciem igitur illustravi de speciminibus Harverans, a v. doct. Wrigirt et $\mathrm{BA}_{\mathrm{A}}$ BINGton Hatodadi et in freto Sangorino lectis, quae vidi et delineavi in herbario Kewensi et de quibus fragmentum ad explorandam structuram anatomicam benevole mecum communicavit v. cl. Orduver, Herbarii Kewensis Conservator.

Frons e basi filiformi saccata, sicca ad $2-3$ (rarius 4 mm.) lata. Tubus centralis adest quidem, sed e segmento transversal $\mathbf{i}$ facile elabitur. Tetrachocarpia ut in ceteris speciebus cruciata, in strato corticali sparsa. Cystocarpia ubique in fronde sparsa semi-exserta, nucleo in stratum periphericum immerso.

\section{GLOEOPELTIS INTIICATA SURIXar l. e.}

Fronde parva intricata pluries dichotoma ramis ex axillis rotundatis patentibus el divaricatis e basi plerumque capillari sensim dilatatis influtis saccato-tubulosis praeter axin centralem racuis, paullum compressis, superioribus denuo tenuioribus et magis magisque approximatis, summis creberrimis capillaribus acutis recurvis saepe alternis ant subsecundis passin bifurcatis ant dentalis.

Frons sicca cornea, madefacta mollissima in partibus inflatis membranacea $2 \frac{1}{2}-4$ rarius ad $6 \mathrm{~cm}$. aita, ad $1 \mathrm{~mm}$. rarius $2 \mathrm{~mm}$. inflata; color fusco-purpureus in speciminibus madefactis ut in toto genere cito expallescit. Fructus aberant. - Tab. XX.

In mari Japonico, I'extor.

Obs. Quamquam hanc spèciem, a tue in Indice praecursorio 1. c. No.69 descriptam, ctiam nunc immutatam servavi, tamen non reticebo dubium, quod interim, inspectis speciminibus authenticis Gl. coliformis HARvEY supra descriptis, mihi obnatum sit, num potius fortasse cum hac specie HArveraxa nostra in unam conjungenda esset. Structura utriusque eadem est, et forma frondis, quamquam in plurimis nostris speciminibus ('lab. XX, fig. $a, f, m$ ) quum ramificatione tum diametro ${ }^{1}$ ) a Harveraxis diversissima, tamen in nonnullis (e. gr. fig. $l, h, i, d$ ) ad harum formam ita accedit, ut haud improbabile milhi videatur fore ut in posterum transitus completus ab una ad alteram formam reperiatur.

1) In comparandis, de figuris nostris, utriusque speciei dimensionibus, animadvertendum est $G l$. coliformen H.nveY (Tab. XLX) de speciminibus siccis delineatam esse, G/. vero nostram intricatam (Tab. XX) de speciminibus madefactis, quae, ut totum genus, aquam avide imbibentes, hoc statu sesquiplicem rel duplicem fere amplitudinem siccorum attingunt. 


\section{EVDOTRTCUIA.}

Fronde vage superne pinnatim ramosa, tubulosa, axe libero monosiphoneo articulato gelineo crasso percursa, ramis ex axe centrali ortis et stratum periphericum frondis sustentantibus (vel potius ramis suis gelineis dense corymbosis poly-dichotomis arcte concretis ipsum hoc stratum periphericum componentibus) illi conformibus patentissimis brevissimis intra spatium pericentrale simplicibus, spatio pericentrali ramulis tenuioribus, plurimis e struto peripherico recurrentibus, rarioribus ex axe ipso egredientibus arachnoideo-repleto, strato corticali gonidionum seriebus moniliformibus dichotomis fastigiatis notato; tetrachocarpiis cruciatis in strato corticali sparsis; cystocarpiis in medium frondis immersis, utrinque paullum prominentibus, placenta dendroidea in axe primario subsessili, nucleo intra spatium pericentrale incluso utrinque strato peripherico integro obtecto (neque in illud immerso), seriebus cellularum sterilibus e centro stralum periphericum petentibus percurso.

\section{ENDOTRICHIA CERTICORNIS. (Suringar l. c.)}

Gloeopeltis cervicornis Suringar, Index praecursorius 1. c. No. 70.

Fronde pusilla pulinata tubulosa compressa, alterne subinde dichotome dense ramosa, ramis ex axillis rotundatis adscendentibus, ramulis supremis dense $2-3$ pinnatis ultimis saepe pectinatis, cystocarpiis in ramulis sparsis in utraque pagina subprominentibus, tetrachocarpiis in strato corlicali sparsis.

Frons sicca cornea dura, madefacta gelatinosa, $1 \mathrm{~cm}$. alta, ad $1 \mathrm{~mm}$. lata. Color fusco-mbens (in Tabulis nostris nimis purpureus pictus est) in madefactis cito ex. pallescit. Superficies nitida - Tab. XXI et XXII.

In mari Japonico, 'I'extor.

Obs. Propter duplicem et frondis et cystocarpii diversitatem hane speciem a genere Gloeopellide separandam et ex illa novum genus constituendum esse duxi. Habitus aliquanto in memorian revocat Endocladiam vernicatam J. AG.; at frondis structura et tetrachocarpia sparsa ab hoc genere abhorrent. Ramulos peculiares, spatium pericentrale tela veluti arachnoidea replentes, nequaquam cum ramis secundariis, quibus in Glocopeltide tenaci spatium pericentrale expleatur, confundendos esse, docent quum sectiones transversales et longitudinales frondis ('Tab. XXII fig. 3, 4) tum frondis fragmenta aqua dulci partim soluta (Tab. XXII, fig. 2, $a, b$ ). Plerique ex ipso strato peripherico in spatium pericentrale recurrunt, alii apicibus liberis hoc spatium pervadunt; alii axem attingentes cum eo concrescunt; rariores ex ipso 
axe oriuntur. Facile tamen et hi a ramis secundariis crassioribus, longius et aequaliter inter se distantibus, stratum periphericum ramis suis tertiariis et quaternariis concretis conformantibus, distinguuntur. Cystocarpium quod in ceteris cum illo Gloeopeltidis convenit, in hoc tamen ab eo discedit, ut nucleus, intra frondis spatium pericentrale ramello axis impositus, ab utraque parte strato peripherico integro obtegatur neque ex uno latere frondis in ipsum illud stratum immersus sit.

\section{GELIDIUM Lamour.}

GELIDIUM CORNEUM (Huds.) IAMOUR.

(Lamodroux, Essai sur les genres de la Famille des Thallassioplyytes non articulés). Kützing, Spec. Alg., pag. 764, J. G. Agardh, Spec. gen. el ord Alg., II, pag. 469. Fucus corneus Hudson (Flora Anglica), 'T'unner, Hist. Fuc., vol. IV, pag. 146, Tab. CCLVII.

In mari Japonico, Siebold et Textor.

Obs. Puncta (gramula) tenuissima quae in Gelidiorum sectione transversali cellulas veluti margaritarum linea cingunt, in sectione longitudinali tenuissima fila esse probantur, quae, longitudinalia in frondis parte inter centrum et ambitum media, ambitum versus transversae excurrunt et ramificantur, ramulis ultimis inter cellulas corticales absconditis.

\section{SPHAEROCOCCUS (Ag. e. p.) KG.}

I. gractlabia Grev.

$$
\text { SPIIAEROCOCCUS (GRACILARIA) CONFERVOIDES (L.) Aø. }
$$

Sphaerococcus confervoides Agardi, Spec. Alg., I, pag. 303. Küтzing, Spec. Alg., pag. 772; Gracilaria confervoides J. G. Agardn, Spec. gen. et ord. Alg. II, pag. 587. - Fucus confervoides L. (Spec. plant, II, pag. 1629), 'I'vrner, Hist. Fuc., II, pag. 30, Tab. LXXXIV.

In mari Japonico, Siebold.

Obs. Specimina sicca alia viridula, alia corallino-rubra fragilissima, haec probabiliter arte picta. 
II. RHOnYMexiA Gret.

\section{SPIIAEROCOCCUS (RIIOD YMENIA) TEYTORII Surtang l. c.}

E basi cuneatä late-linearis plana coriacea, e margine allerne ramosa et subdichotoma, ramis ramulisque sursum angustutis apice productis sive acutiusculis, cellulis frondis medullaribus maximis, cystacurpiis per frondis superficiem sparsis exsertis.

Specimina sicca expallida $15 \mathrm{~cm}$. longa, laciniis ad $1 \mathrm{~cm}$. latis. - Tab. XXIII.

In mari Japonico, T'extok.

Obs. Proxima Rhodymeniae palmatae (L.) Grév., at firmior, crassior. Memoratu dignae sunt cellulae in ipso strato corticali cystocarpium tegente observatae, (fig. 3, 7) simplices vel divisae, tetrachocarpiis valde similes.

\section{GYHNOGOMGRUS MART,}

I. oxcotrue KG.

GYMNOGONGRUS (ONCOTYLUS) FLABELLIFORIILS HARVE.

Harver, Algne in: List of dried p'ants collected in Jupan by S. WELLS WIILAMus Esq. and Dr. JuMes Marrow. (decount of the botanical specimens prepared by Prof. A. Grax assited by Mr. Sulituant, Dr. Harver, cet. 1. 1. pag. 331), No. 17 .

"Fronde pumila compresso-plana angusta linenri pluries dichotoma ambitu flabelliformi segmentis apice vix dilalatis fastigiatis obtusis vel emaryinalis.

Not two inches high, resembles a small specimen of the narrow varieties of Chondrus crispus." Hakver l. c.

In mari Japonico, 'I'extor. - 'I'ab. XXIV, B.

Obs. Specimina Harveraxa non vidi, hine determinatio dubia. Nostra madefacta carnosa usque ad $7 \mathrm{~cm}$. alta, laciniis ad $3 \mathrm{~mm}$. latis. Color violaceo-purpureus. Fructus aberant.

GYMNOGONGRUS (ONCOTYLUS)? JAPONICUS Suringar 1. c.

Fronde gracili membranacen iterato dichotoma flabellata protifera, lacinïs angustissime linearibus compressis superne paullum dilatatis complanatis, apicibus obtusis aut furcalis. 
$5 \mathrm{~cm}$. alta; laciniae versus apices vix $1 \mathrm{~mm}$. latae. Color in inferiore parte nigrescens, in prole juniori fusco-purpurascens. - Fructus non vidi. - 'Tab. XXIV, A.

In mari Japonico, 'I'Extor.

Obs. Habitus Dicureilae, structura Gymnogongri; propter fructus ignotos genus dubium.

\section{POLYSIPIIONIA GRÉv.}

\section{POLYSIPHONta fragllis Suringar 1. c.}

Parva (pollicaris) nigrescens alterne ramosa, ramis ramulisque rigidiusculis patentibus vel varie divergentibus, apicibus bifurcatis nudis, articulis diamelro (ad $0,2:=\frac{1}{11}^{\prime \prime \prime}$ ) ad $\frac{2}{3}$ brevioribus 5-siphoneis, siphone centrali tenui. Fructus non aderant. - 'Tab. XXV, B.

In mari Japonico, von Srebold.

Obs. P. spinellae Ag. aftinis videtur.

\section{POLISIPHONIA TAPINOCARPA SURINGar l, c.}

Spithamea flaccidu mucosa intricala fusco-purpurea iterato alterne ramosa ramulis penicillatim confertis, upice pulchre fibrosis, tetrachocarpiis in articulis ramulorum penultinorum et ante-penultimorum tumidis seriatis, articulis hom ramorum diametro $\left(0,04 \mathrm{mu} .=\frac{1}{56}{ }^{\prime \prime \prime}\right)$ duplo longioribus 9-siphoneis siphone centrali ceteris conformi, inferionem crassioribus longioribus, infunis corticatis. - 'Tab. XXV, A.

Obs. Affinis videtur $P$. atrorubenti Grév. et $P$. bipinnatae Post. et Rupr.

\section{DIGENEA AG.}

\section{DIGENEA WULFENI KG.}

Küтzing, Plyycologia generalis, pag. 433, Tab. 1, Spec. Al\%., pag. 841, Digenea simplex (Wulf.) Agardi, Spec. Alg., I, pag. 389, Jac. G. Agardi, Spec. gen. et ord. Alg. II, pag. 845. Conferva simplex Wulfes, Cryplogamia aquatica, pag. 17.

In mari Japonico, Textor. 


\section{INDEX SPECIERUM.}

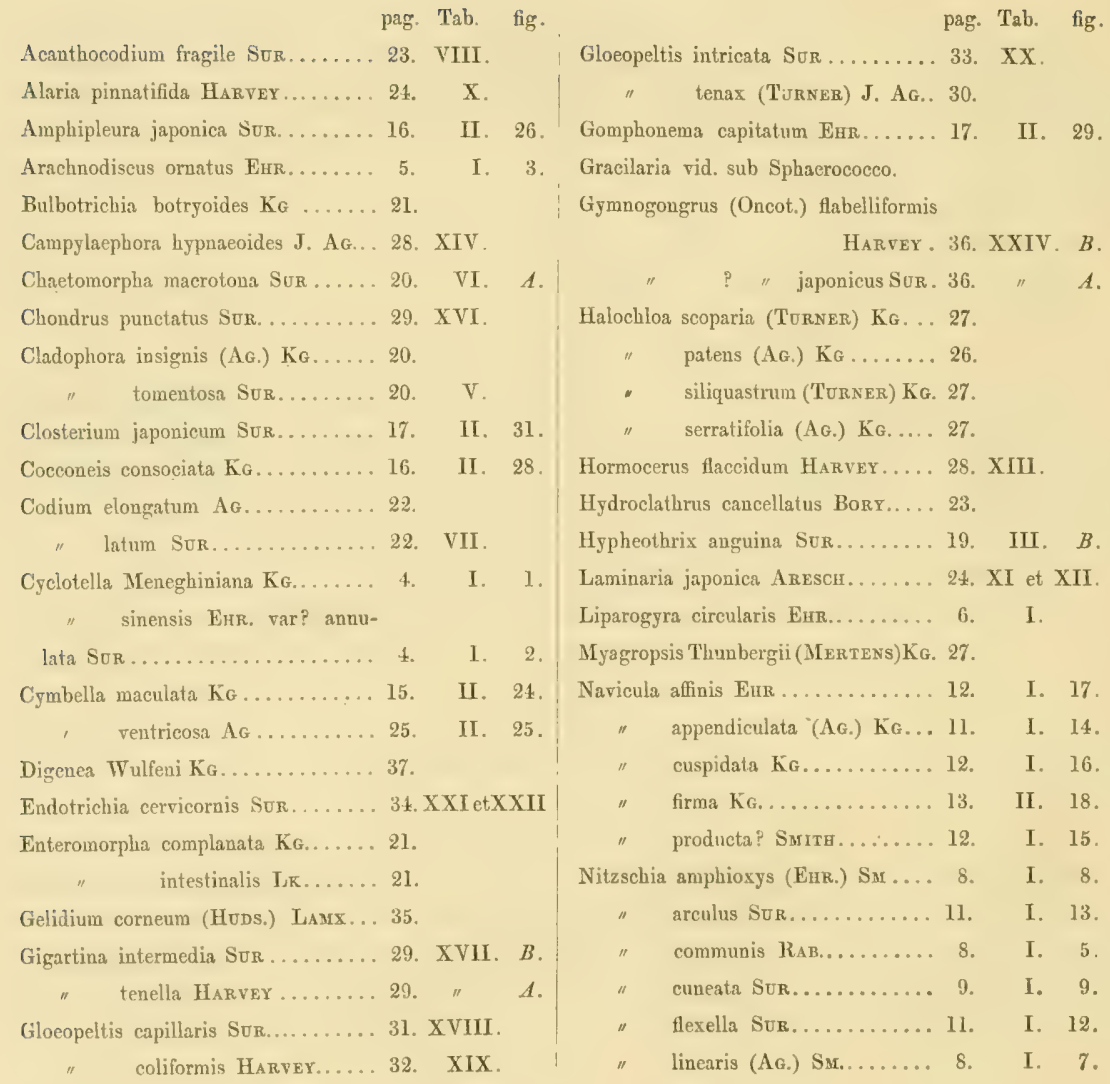


pag. Tab. fig. I

Nitzschir palea (KG.) Sr......... 10.

" sigmoider (Nitsch) S... S.

" tenuis Sщтt........... 10.

Oncotylus vid. sub Gymnogongro.

Phycoseris australis $\mathrm{K} G . . . \ldots \ldots . .21$.

Phyllitis debilis (AG.) KG.......?

Pinnularia maior (KG.) Sx. ....... . 1 viridis (Nitzsch) SM.... 14. II. 20.

Pleurosigma Spencerii Suпн f..... 1 1.

Polysiphonia fragilis SUR. 3i. $\mathrm{XXV}$.

tapinocarpa SoR...... 37. XXV.

Porphyra vulgaris AG........... dy.

Rhodymenia vid. sub Sphrerococco.

Rlioicosphenia curvata ( $\mathrm{K}$ G. $)$ Grüsow. 17

I. 10 .
I. 6. Schizymenia? ligulata SuR........29. XV.

1. 11. Scoliopleura ornata (KG. ?) SuR.... 14. II. 22 . Sirosiphon vermicularis Str. ..... 19. III. 1 .

Spathoglossum multipartitum(SшнR)KG,28. IX. $t$.

Sphaerococcus (Gracilaria) confervoides (L.) AG....... 35.

(Rhodym.) Textorii Sur, 30. XXIIJ.

Spirogyra lineata Sur........... Is. IV.

Spongocarpus hemiphyllus (T'URN.) KG. 26.

1. "Horneri (TURNER) KG. 26.

" Sisymbrioides (TURN.) KG. 26. Stauroneis Fenestra EHr......... 15. II. 23.

II. 30. Tetracyclus lacustris RaLFs...... 16. II. 27. 
T A BULAE. 


\title{
EXPLICATIO T'A B ULARUM.
}

\author{
TAB. I.
}

\section{DIATOMACEAE.}

Fig. 1. Cyclotell $\alpha$ Meneghiniana KG. $a, a^{\prime}:$ a facie valvari; $b$ : a latere connexivo. $490 \times \mathrm{d}$.

" 2. Cycotella sinensis EHr. var.? C. annulata Sur. a facie valvari. $490 \times \mathrm{d}$.

" 3. Arachnodiscus ornatus Eur. a facie valvari superiore. $490 \times \mathrm{d}$.

" 4. Liparogyra circularis Eur. em. $a: 219 \times \mathrm{d} ; b-g: 190 \times d ; a-f:$ a latere, $g$ : oblique a fronte visa.

"5. Nitzschia communis RAB, $490 \times d$.

"6. Nitzschia sigmoidea (Nitzscr) Smith, $490 \times \mathrm{d}$.

"7. Nitzschia linearis (AG.) SмrтH, $490 \times \mathrm{d}$.

" 8. Nitzschia amphioxys (EнR.) Sмгти, $a-e: 490 \times \mathrm{d} ; e^{\prime}: 860 \times \mathrm{d} ; a$ : a látere connexivo; $d$ : a valva; $b, c, e, e^{\prime}$ : oblique, a facie carinali.

" 9. Nitzschia creneala Sur. $490 \times \mathrm{d}$.

"9† eadem species de speciminibus Hollandicis. $a-l: 490 \times \mathrm{d} ; l^{\prime}, l^{\prime \prime}: 860 \times \mathrm{d} ; a, c, f:$ a latere connexivo; $b, e, g, h, l, l^{\prime \prime}:$ a facie valvari; $d, i$, ,carina" superne versa; $k:$,carina" laterali.

"10. Nitzschia palea (KG.) Sмiтu, $490 \times \mathrm{d}$.

" 11. Nitzschia tenuis Smith, var. parva RAB. $490 \times$ d.

" 12. Nitzschia flexella Sur. $490 \times$ d.; $a-c$ : a valvis, $f, g$ : obliqua jacens .carina" superne versa; $e, h$ : „carina" laterali.

"13. Nitzschia arculus Sur. $430 \times \mathrm{d}$.

" 14. Navicula appendiculata KG. $490 \times \mathrm{d}$; a valvn; $a, b$ : re lanceoluta Grün.; $c$, $d$ : $\beta$ exilis GrüN.

" 15. Navicula producta Surir? $490 \times d$; a facie valvari.

"16. Navicula cuspidata KG. $490 \times \mathrm{d} ; a-c$ : a facic valvari. $d, e$ : ejusdem speciei forma longicollis SuR. $d$ : a latere connexivo e: a valva. $490 \times d$.

"17. Navicula affinis Err. a facie valvari.

" $17 *$ ejusdem speciei? forma minor, a facie valvari.

\section{TAB. II.}

\section{DIATOMACEAE.}

Kig. 18. $a-d, l, m:$ Navicula firma KG. a maior Griinow. $419 \times \mathrm{d}: a, b, l, m: a$ valva; $c$ : obliqua jacens. angulo inter valvam et latus connexivum superne verso: $d$ : obliqua, sed magis valvam versus. Positum indicant sectiones transversae schematicae singulis figuris subscriptae.

$e-h$ : ejusdem forma triunduiata SuR. $e-h: 219 \times \mathrm{d}$; $e:$ a latere connexivo, $f-h:$ a valvis; $i, k: 490 \times d ; i:$ a valva; $k$ : a latere connexivo.

" 19. Pinnularia major (KG.) $a-c: 219 \times \mathrm{d} ; a-c: 490 \times \mathrm{d} ; a, d:$ a latere connexivo; $b, c, e, f: a$ valva.

" 20. Pinnularia viridis Eur. $a: 490 \times \mathrm{d} ; \quad b, c: 219 \times \mathrm{d} ; a, b: a$ valva; $c: a$ latere connexivo.

" 21. Plearosigma Spenceri Sмitn? $490 \times \mathrm{d} ;$ a ralva. 
Fig. 22. Scoliopleata omula Sok. $a-e: 219 \times \mathrm{d} ; d-f: 490 \times \mathrm{d} ; b, d: a$ latere connexiro; $c, e: a$ ralva; $a, f$ : obliqua jacens in uno latere ralvae. Positum indicat sectio transversalis schematica fig. $e$ subscripta; lineis punctatis ex hac sectione sinistrorsin et dextrorsim directis latera figurarum $d$ et $f$ indicantur.

Cum tigura a comparetur figura Kützingiana Surirella? suac ornatae in opere: Bacillariën, Tab.III, fig. 54. 23. Siauroneis fenestra Eur. $490 \times \mathrm{d}$; a valva.

"24. Cymbella maculata KG. $490 \times$ d; $a, b, d$ : a latere connexiro; $c$ : a valva.

" 25. Cymbella ventricosa KG. $190 \times \mathrm{d}$; a latere connexivo.

" 26. Amphipleura jayonica SUR. $490 \times \mathrm{d}$; $a$ : oblique a latere connexivo. In valvarum marginibus strine tenuissimne transversae conspiciuntur. $b$ : a latere ralrari, plicas tres ostendens.

27. Tetracyclus lacustris RALFs? tantum a latere connexivo observata. $219 \times \mathrm{d}$.

"28. Cocconeis consociata KG. a ralra, 9S0 (nou 19u) $\times$ d.

29. Gomphonema capilatum Eur. $490 \times \mathrm{d} ; c-d$ : a latere connexiro; $a, b, e$ : a valva.

"30. Rhoicosphenia curvatu (KG.) GRüNor, $490 \times \mathrm{d}$; $a$ : a latere connexiro; $b$ : a latere valvari.

1) ESILIDI.ICE.IE.

" 31. Closterium japonicum Sur. $a: 219 \times \mathrm{d} ; \dot{i}, c, d: \pm 90 \times \mathrm{d} ; c:$ strias longitudinales ostendens.

TAB. III.

A. SIROSIPHON VERMICULARIS SUR, $219 \times$ d.

B. HYPHEOTHRIX ANGUINA SUR.

$219 \times$ d. Fig. : pars media et apicalis trichomatis, $450 \times d$.

TAB. IV.

Fig. 1. Pars strati.

SPIROGYRA LINEATA SUR.

" 2. Filamenta singula. $50 \times$ d. Litterae $a, e, i, h$, erolutionis gradus dirersos indicantes, cum iisdem in fig. 3 conveniunt.

" 3. $a-i$ : Partes diversae ejusdem filamenti ab apice juniore $(a)$ retrorsum procedentes, $219 \times \mathrm{d}$.

$a, a^{\prime}, a^{\prime \prime}$ : Cellulae cum fasciis chlorophyllophoris primariis.

$b$ ad $c$ : Fasciae chlorophyllophorae primariae sensim dissolvuntur et in stratum fibrarum decolorum convertuntur.

$d$ : Intra stratum striatum norae nascuntur vesiculae chlorophyllophorae.

$e$ : Vesiculae chlorophyllophorae numero auctie, in series concatenatae.

$f$ : Incremento et divisione vesicularum chlorophyllophorarum citius quam cellularum ipsarum incremento procedente, series initio simplices ramificantur.

g: Denique e ramis hisce sertiformibus fasciae fere spiraliformes, primariis non dissimiles oriuntur.

$h$ : Fasciae chlorophyllophorae secundariae sua vice protrahuntur et dissolvuntur.

$i$ : Status aduitus, ubi chlorophyllum sensim dissolvi et renorari videtur sine conspicua formae mutatione.

$i^{\prime}$ : Pars similis alius filamenti.

TAB. V.

Fig. 1. Pars caespitis.

CLADOPHORA TOMENTOSA SUR.

"2. Trichomata singula, $50 \times$ d.

" 3. Partes inferiores, mediae et apicales trichomatis, $219 \times \mathrm{d}$. 
TAB. V1.

Fig. 1. Pars caespitis.

A. CHAETOMIORPHA MACRÓtONA SUR.

2. Filamentum, $50 \times$ d.

B. PHYCOSERIS AUSTRALIS KG.

"1. Specimen integrum.

"2. Sectio frondis transversa, $219 \times \mathrm{d}$.

"3. Frons superne visa, $219 \times \mathrm{d}$.

TAB. VII.

Fis: 1. Specimen integrum.

CODIUM LATUM SUR.

2. Sectio transversa frondis, $50 \times d$.

" 3. Ejusdem pars, $215 \times$ d. In utraque figura cellulae periphericae, in parte inferiori collapsae, reluti pedicellatae apparent.

" $t$. Cellulae periphericae a superficie frondis solutae, $50 \times \mathrm{d}$.

" 5. Eaedem, $215 \times$ d. Juxta basin cellularum periphericarum in cellulis frondis tubulosis conspiciuntur dissipimenta illa crassissima medio canali exiguo perforata.

TAB. VIII.

Fig. 1. Fragmenta frondis.

ACANTHOCODIUIT FRAGILE SUR.

"2. Sectio transversa froudis, $50 \times \mathrm{d}$.

" 3. Sectio longitudinalis frondis, $50 \times \mathrm{d}$.

" 1 . $a-c$. Cellulae periphericae a fronde solutae.

TAB. IX.

Fis. 1. Frons integra.

A. SPATHOGLOSSUM MULTIPARTITUM (SשHR) KG.

2. Eroudis sectio transversa, $215 \times$ d.

3. Pili a basi frondis, $215 \times \mathrm{d}$.

1. Frondes integrae.

PHYLLITIS DEBILIS (AG.) KG.

"2. Sectio frondis transversa, $215 \times \mathrm{d}$; frons superne visa, $215 \times \mathrm{d}$.

TAB. X.

ALARIA PINNA'TIFIDA HaRvey.

Fig. 1. Frons integra, magnitndine naturali.

STATU JUNIORI.

"2. Alia frons perpusilla magnitudine naturali.

" 3. Frondis superficies, $50 \times \mathrm{d}$.

" $\quad+$ - 6. Sectiones transversae frondis $4,5: 219 \times \mathrm{d} ; 6: 50 \times \mathrm{d}$. 
TAB. XI.

LAMINARIA JAPONICA Areschovgh,

Specimina ad septimam maguitudinis partem reducta.

TAB. XII.

LAMINARIA JAPONICA AREschodgh.

Fig. 1. Basis frondis cum stipite et rhizomio, magnitudine naturali.

" 1. $a, b$ : Aliorum speciminum adumbrationes inferioris partis.

"2. Sectio transversa frondis, $215 \times \mathrm{d}$.

TAB. XIII.

HORMOCERAS FLACCIDUM HaRtey.

Fir. 1. Specimen integrum magnitudine naturali.

2. Specimen integrum, $27 \times \mathrm{d}$.

"3. Pars apicularis, $130 \times \mathrm{d}$.

" 4. Pars trichomatis primarii, $130 \times \mathrm{d}$.

TAB. XIV.

CAMPYLAEPHORA HYPNAEOIDES J. Ag.

Fig. 1. Specimen fere integrum, magnitudine naturali.

"2. Apices uncinati ramorum, $2 \times \mathrm{d}$.

"3. Sectio trausversa trichomatis. $50 \times \mathrm{d}$.

" 3 a. Ejusdem dimidia pars, $219 \times \mathrm{d}$.

" \$. Sectio longitudinalis trichomatis.

" 4 a. Ejusdem dimidia pars, $219 \times \mathrm{d}$.

Obs. Color corallino-ruber artificialis est, ut etiam in aliis Algis japonicis cibariis.

\section{TAB XV.}

SCHYZIMENIA LIGULATA SURINGAR.

Fig. 1. $a-e$ : Frondes integrae, magnitudine naturali.

2. Sectio transversa frondis. $50 \times d$.

") 3. Sectio transversa frondis, $215 \times \mathrm{d}$.

\section{TAB. XVI.}

\section{CHONDRUS PUNCTATUS SURINGAR.}

Fig. 1. Frous integra, magnitudine naturali, inferne cystocarpius punctiformibus dense obsita. "1a. Pars frondis sterilis.

"2. Sectio transrersa frondis fertilis per cystocarpium ducta, $61 \times \mathrm{d}$.

" 3. Sectio transversa tenuissima cystocarpii, $300 \times$ d.

" 4. Sectio transrersa frondis, $215 \times d$. 
TAB. XVII.

A. Gigartina tenella Harvey.

Fig. 1. Frondes integrae, magnitudine naturali. $\alpha, d$ : madefactre; $c, e, b$ : siccae.

2. Sectionis transversae frondis dimidia pars, $219 \times \mathrm{d}$.

Ubs. Frons desiccata fere ad $\frac{2}{3}$ magnitudinis frondis madefactae contrahitur.

\section{B. GIGARTINA INTERMEDIA SURINGAR.}

Fig. 1. Frons integra magnitudine naturali (sicca).

2. Sectio transversa frondis in rami apice dilatato et sulcato, $50 \times \mathrm{d}$.

" 3. Sectionis transversae frondis dimidia pars, $219 \times \mathrm{d}$.

TAB. XVIII.

GLOEOPELTIS (COELORELTIS) CAPILLARIS SuRINGar.

Fig. 1. Frondes integrae magnitudine naturali.

" 2. Sectio transversa infimae partis frondis, ubi spatium inter axem centralem et stratum periphericum omnino evaniduı. $290 \times$ d.

"3. Ejusdem forma rarior, ubi axis ramis irregularibus oblique in stratum periphericum adscendentibus cingitur, $219 \times \mathrm{d}$.

4. Eadem ac fig. 2; sed ubi circa axem spatium pericentrale perspicuum est, $219 \times \mathrm{d}$.

5. Sectio transversalis frondis e superiore parte cum tetrachocarpiis, $219 \times \mathrm{d}$.

"6. Ejusdem sectio longitudinalis, ubi axis alterne ramosus, $60 \times d$.

"7. Eadem, $219 \times \mathrm{d}$; ad demonstrandum ramorum decursum per stratum frondis periphericum.

" 8. Sectio longitudinalis frondis cum cystocarpii, $(a) 60 \times \mathrm{d} ; a^{\prime}, a^{\prime}, a^{\prime \prime}, a^{\prime \prime \prime}$ : partes placentre dendroideae centralis cum sporarum seriebus levi pressione solutis.

TAB. XIX.

GLOEOPELTIS (COELOPELTIS) COLIFORMIS HARVEY.

Fig. 1. $a-g$ : Specimina integra (sicca) magnitudine naturali; secundum specimina authentica in Herb. Kercensi a me delineatr.

"2. Sectio transversa frondis sterilis, $57 \times \mathrm{d}$.

"3, 6. Ejusdem pars, $215 \times$ d.

" 4. Sectio transversa frondis cum cystocarpiis, in stratum periphericum immersis, $215 \times \mathrm{d}$.

" 5. Sectio eystocarpii, $215 \times \mathrm{d}$.

"7. Sectionis transversae pars cum tetrachocarpiis.

\section{TAB. XX.}

GLOEOPELTIS (COELOPELTIS) INTRICATA SUR.

Fig. 1. $a-0$. Specimina (madefacta) magnitudine naturali; $a, f, m$ : typica integra; $b, c, e, g$ : fragmenta ramorum apicalia; $l, n, o, h, i$ specimina sensim ad formam simpliciorem aequalius dilatatam procedentia; $d$ : fragmentur apicale ample tubulosum cum prole capilliformi.

"2. Sectio transversa frondis in parte inflata rami; $a: 50 \times \mathrm{d} ; b$ : ejusdem pars, $215 \times \mathrm{d}$.

" 3, 4. Sectiones transversae frondis in partibus capillaribus. $3 b c, 4 a: 50 \times \mathrm{d}: 3 a, 4 a: 215 \times \mathrm{d}$. 


\section{TAB. XXI.}

\section{ENDOTRICHIA CERVICORNIS SURINGAR.}

Fig. 1. $a, b, c, d$. Specimina integra sicea, magnitudine naturali.

1. e. Ramus (madefactus) cum cystocarpiis, $3 \times \mathrm{d}$.

" I. $f$. Specimen parrum (madefactum) cum tetrachocarpiis, $3 \times$ d.

" 2. Apex rami a specimine tetrachocarpia gerente. Puncta quae in superficie sparsa apparent, tetrachocarpia sunt. $14 \times \mathrm{d}$,

3. 4. Apex rami a specimine cystocarpia gerente $14 \times \mathrm{d}$.

5. Placenta e fronde soluta, cum axe centrali, in quo subsessilis, $215 \times \mathrm{d}$.

" 7. Sectio frondis transversa per cystocarpium, $215 \times \mathrm{d}$.

" 6. Eadem, sporis levi pressione solutis; conspicitur placenta centralis transverse dissecta cum ramis, inter sporas, ex ea excrescentibus et stratum periphericum sustentantibus.

\section{TAB. XXII.}

\section{ENDOTRICHIA CERVICORNIS SURINGAR.}

Fig. 1. $a$ : Frons transterse, $b$ : longitudinaliter dissecta, $60 \times \mathrm{d}$.

" 2. Pars phycomatis aqua dulci et levissima pressione partim soluta. $a: 57 \times \mathrm{d} ; b: 215 \times \mathrm{d}$; in superiore parte figurae $2 \alpha$. bifurcatio axis (in ipsius frondis bifurcatione) conspicitur; in inferiore parte apparent rami penecillatim congesti, qui in fronde solide concreti ejus stratum periphericum constituunt. Fig. $b$ : eadem inferior pars magis aucta, ad illustrandum originem ramellorum a pariete frondis interna introrsum decurrentium.

"3. Sectio transversa frondis cum tetrachocarpiis.

" 4. Sectio longitudinalis frondis juxta axem centralem, cujus fragmentum in inferiore et in suporiore parte figurae conspicitur.

\section{TAB. XXIII.}

\section{SPHAEROCOCCUS (RHODYMENIA) TEXTORII SURINGAR.}

Fic. 1. Frons integra cystocarpia gerens (sicca) magnitudine naturali.

"2. Alius speciminis fragmentum.

"3. Sectio cystocarpii, $57 \times \mathrm{d}$. Perspicuus est placenta centralis basilis. In strato corticali lacunae conspiciuntur; unde de cellulae peculiares, fig. 7 plenius illustratae, elapsae sunt.

" + Sectio frondis transversa, $57 \times \mathrm{d}$.

" 6. Eadem inde a superficie usque ad frondis mediam partem $215 \times \mathrm{d}$.

5. Ostiolum cystocarpii, $215 \times \mathrm{d}$.

. 千. Pars strati corticalis cystocarpii cum cellulis illis peculiaribus simplicibus aut in duas divisis, tetrachocarpiis similibus, $215 \times \mathrm{d}$.

"S. Series cellularum sporigenarum e placenta erolutae, $215 \times \mathrm{d}$. 
TAB. XXIV.

\section{A. GYMNOGONGRUS (ONCOTYLUS)? JAPONICUS SUR.}

Fig. 1. Frons integra.

"2. Ejusdem pars terminalis.

3. Sectio transversa frondis, $215 \times \mathrm{d}$.

4. Sectio longitudinalis frondis. $215 \times \mathrm{d}$.

\section{B. GYMNOGONGRUS (OACOLYLUS) FLABELLIFORMIS HARYEY.}

Fig. 1. Speciminum integrum madefactum.

" 2, 3. Specimina sicca.

" 4. Sectio transversa frondis, $50 \times \mathrm{d}$.

" 5. Sectio longitudinalis frondis, $215 \times \mathrm{d}$.

TAB. XXV.

\section{A. POLYSIPHONIA TAPINOCARPA SURINGar.}

Fig. 1. Pars caespitis magnitudine naturali.

"). Rami singuli.

" 3. Unus ex is $3 \times$ d. auctus.

" 1. Apex trichomatis cum tetrachocarpiis, $60 \times \mathrm{d}$.

". .. Pars rami antepenultimi cum tetrachocarpiis; $b$. $e$ : tetrachocarpia ex articulis exempta, $60 \times \mathrm{d}$.

" (i, 8. Partes inferiores trichomatis, articulis elongatis, $60 \times \mathrm{d}$.

"7. Probabiliter pars trichomatis primarii corticati, $60 \times \mathrm{d}$.

" !. 10. Sectiones transrersae trichomatis.

"11. Apex ramuli, $219 \times$ d.

\section{B. POLYSIPHONIA FRAGILIS SURINGAR.}

Fig. 1. $a-f$ : Specimina sicea magnitudine naturali.

" 2. Pars inferior trichomatis, $60 \times \mathrm{d}$.

" 3. Pars apicularis trichomatis, $60 \times \mathrm{d}$.

" 1.5 . Sectiones transversae trichomatis. 
$\ldots$,

......

; $\cdots$

, ...

if

.. 1.

; i ' : ' :

$\therefore$. . . .

i) , * .

,....
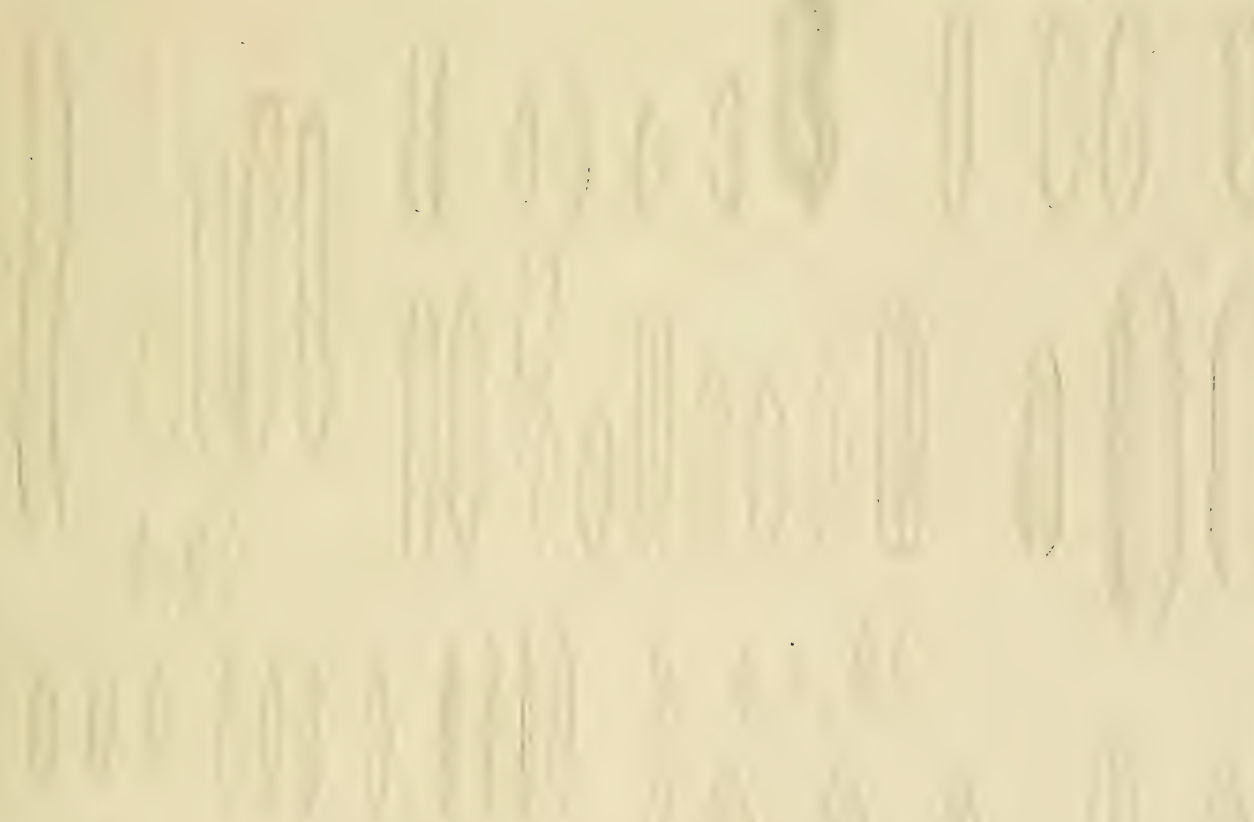

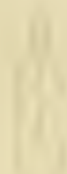




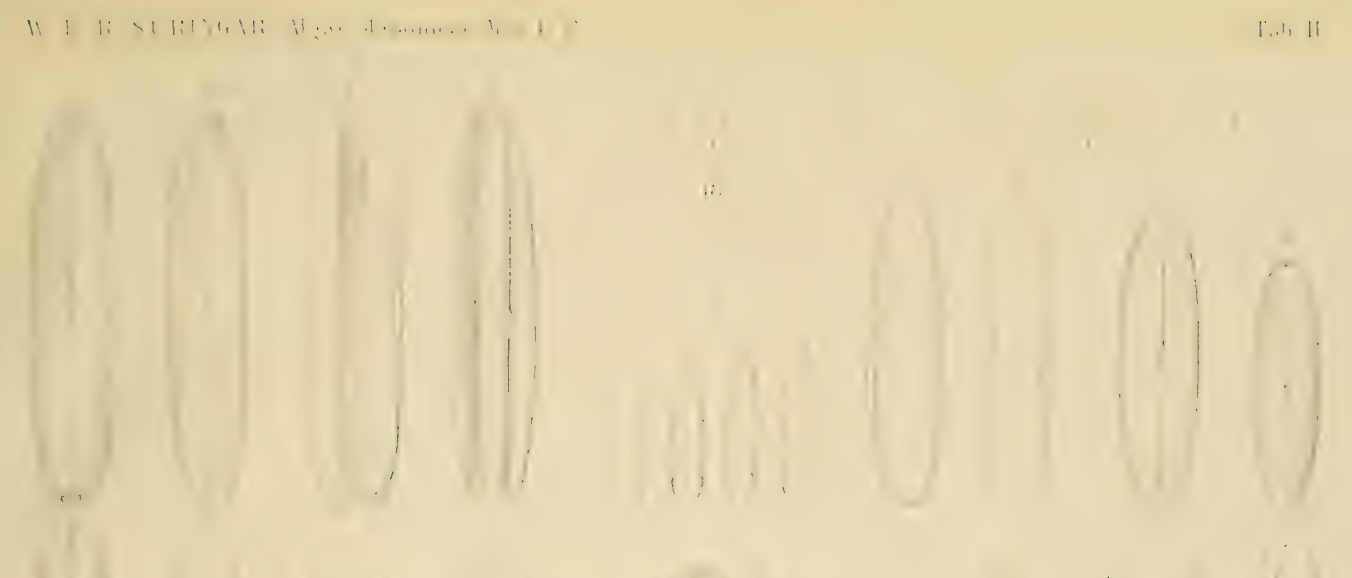

(11)

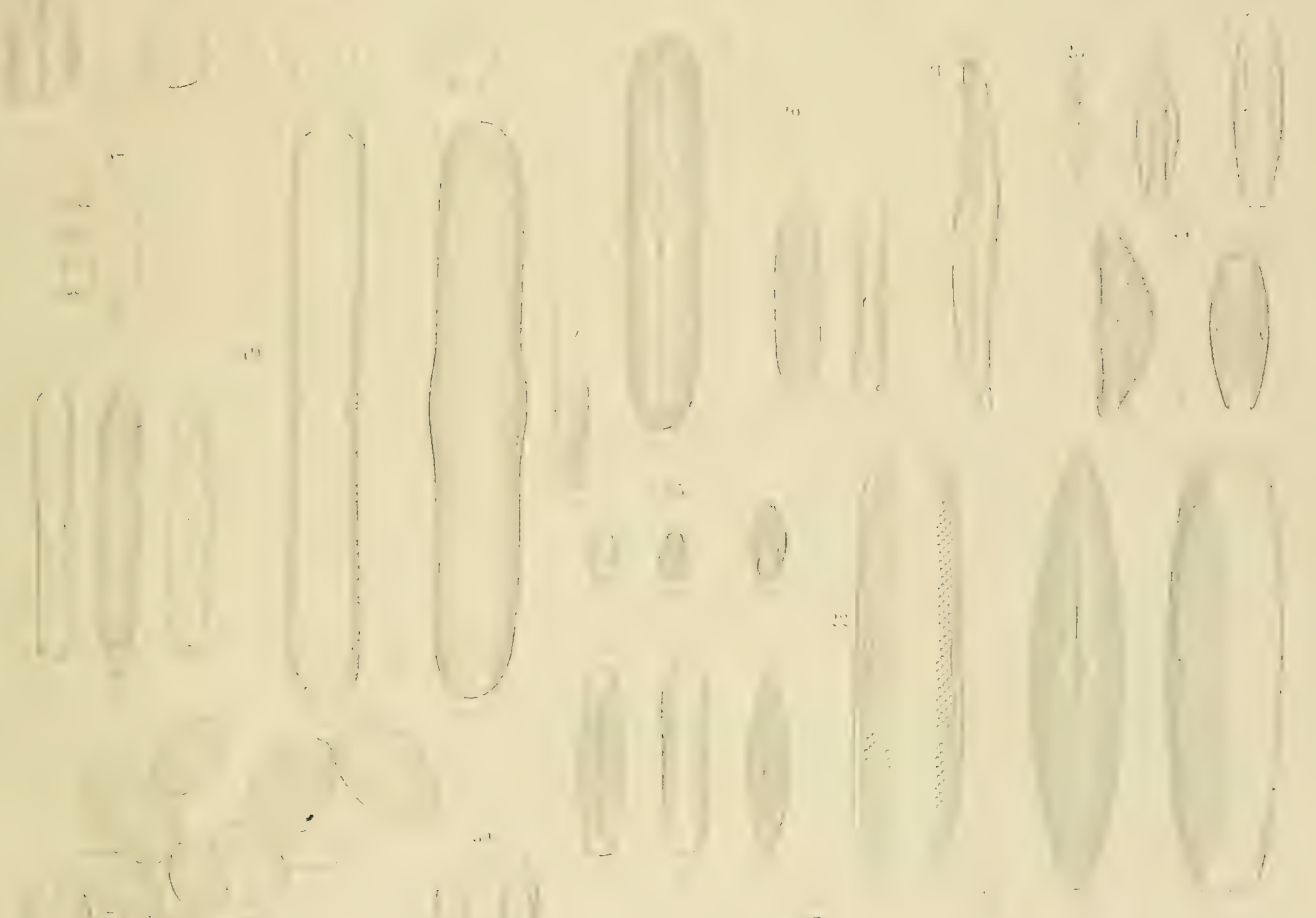

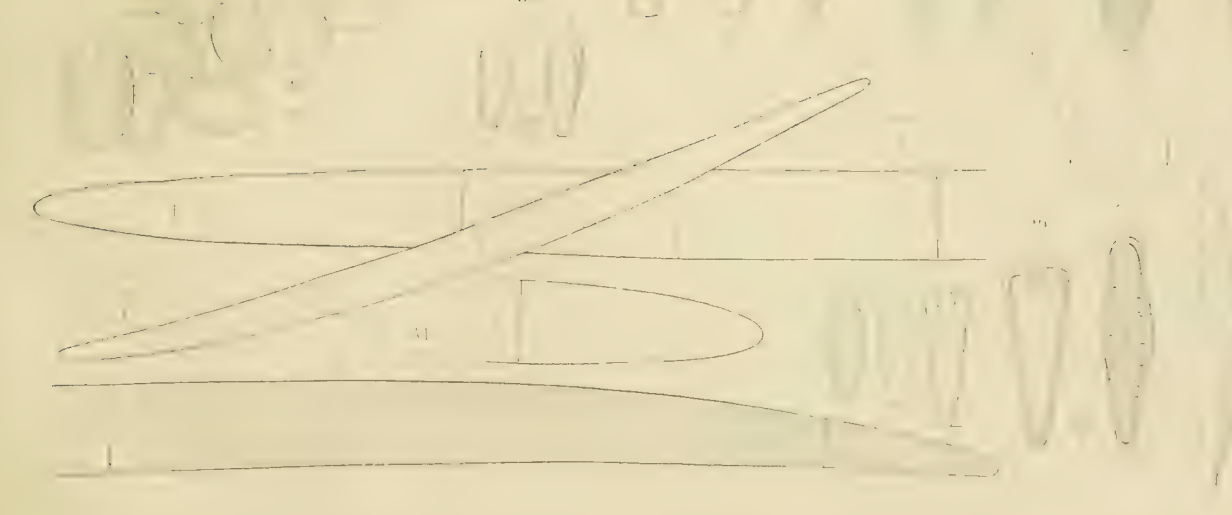




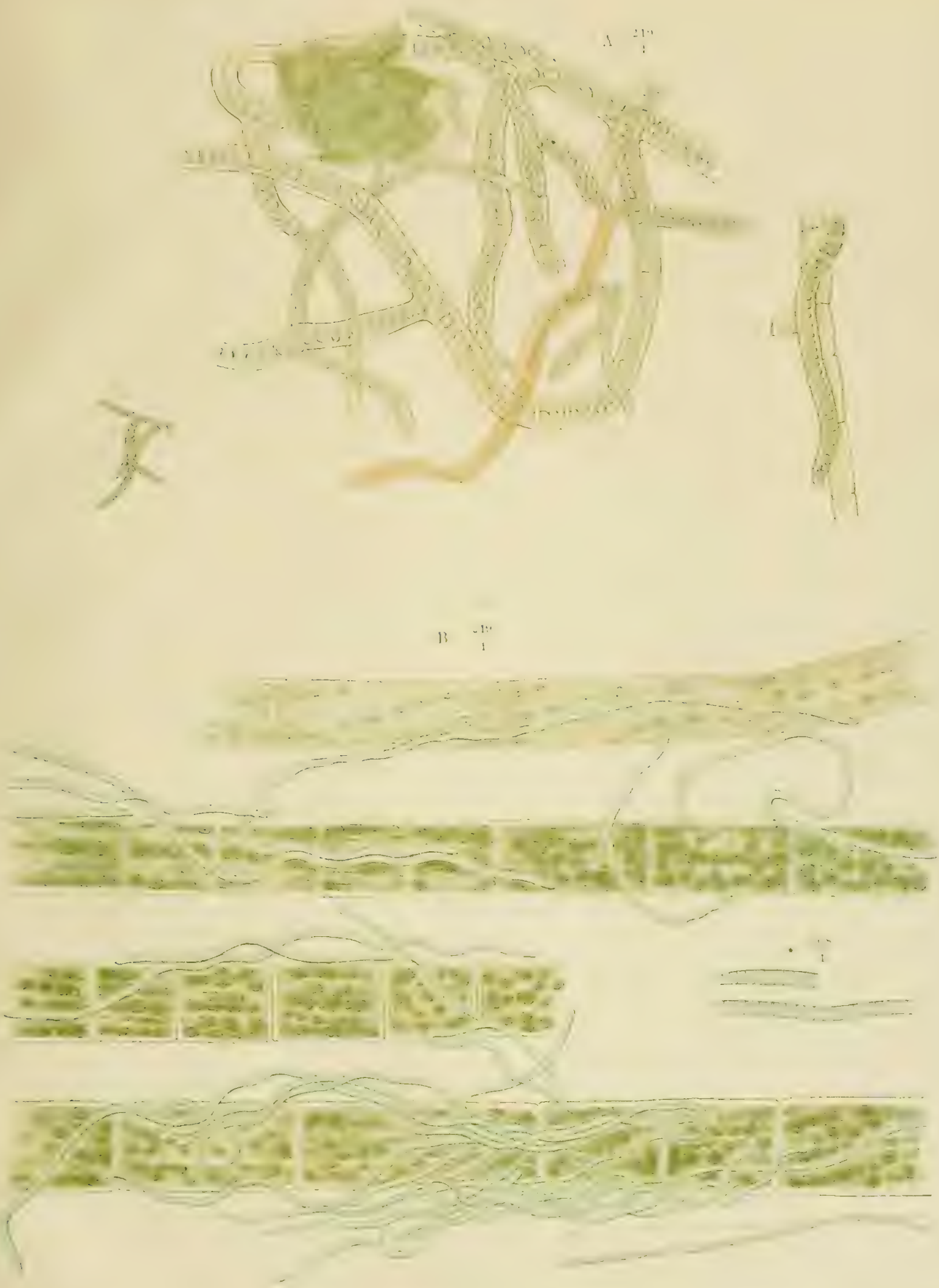


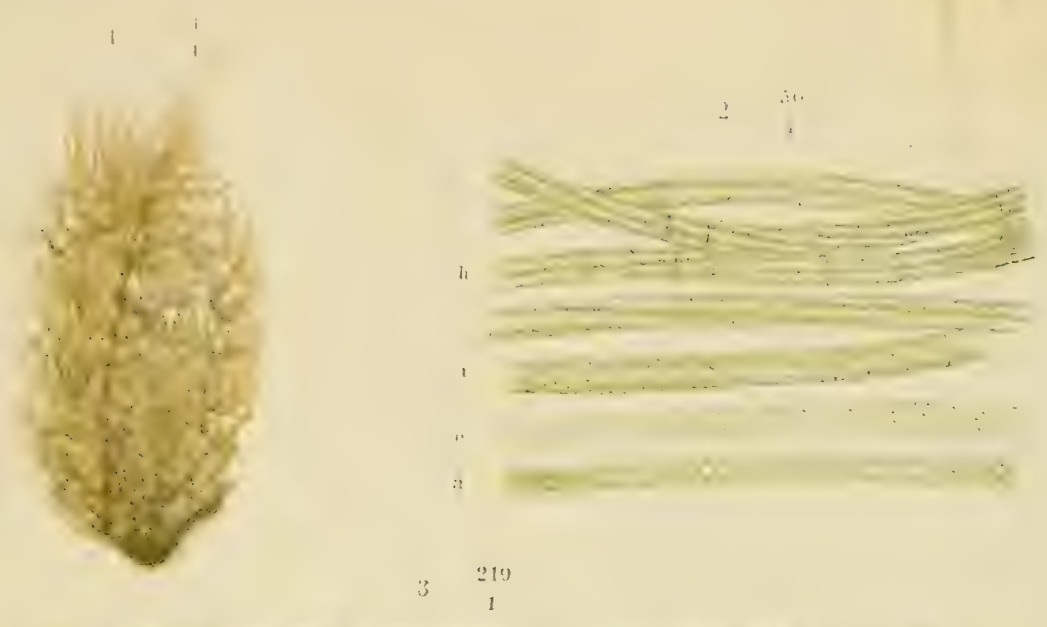
a
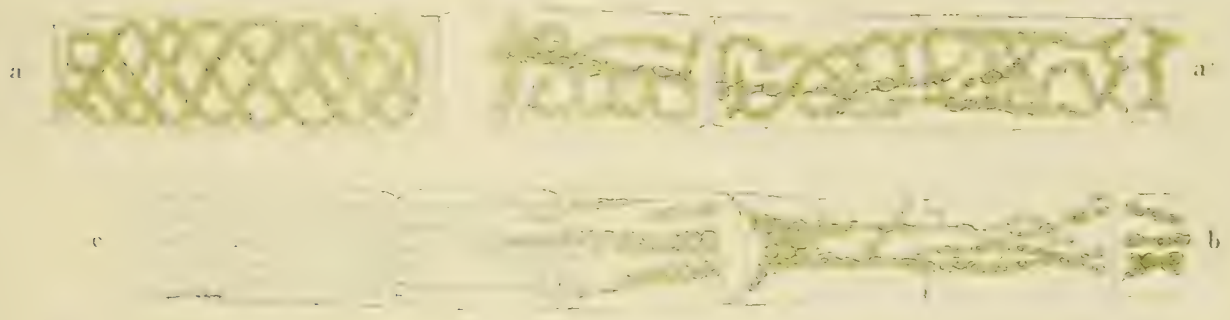
$\frac{2+\cdots+\infty}{4+\infty}+\cdots$

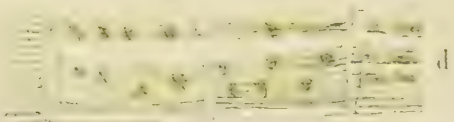

B.

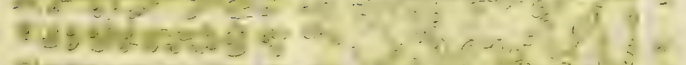

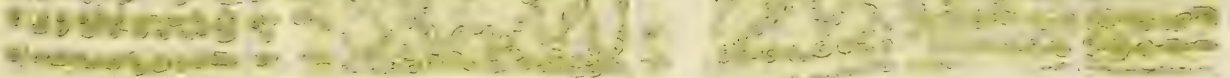

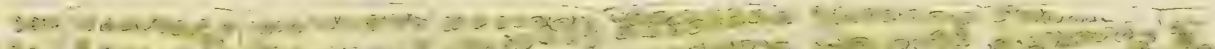

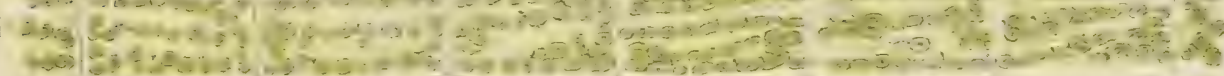

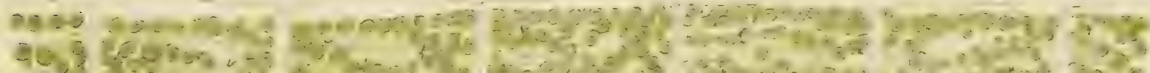

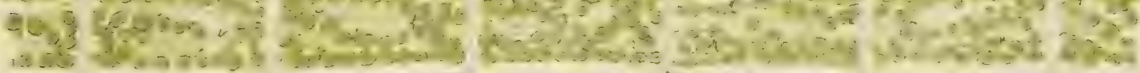


$$
\text { , }
$$ 

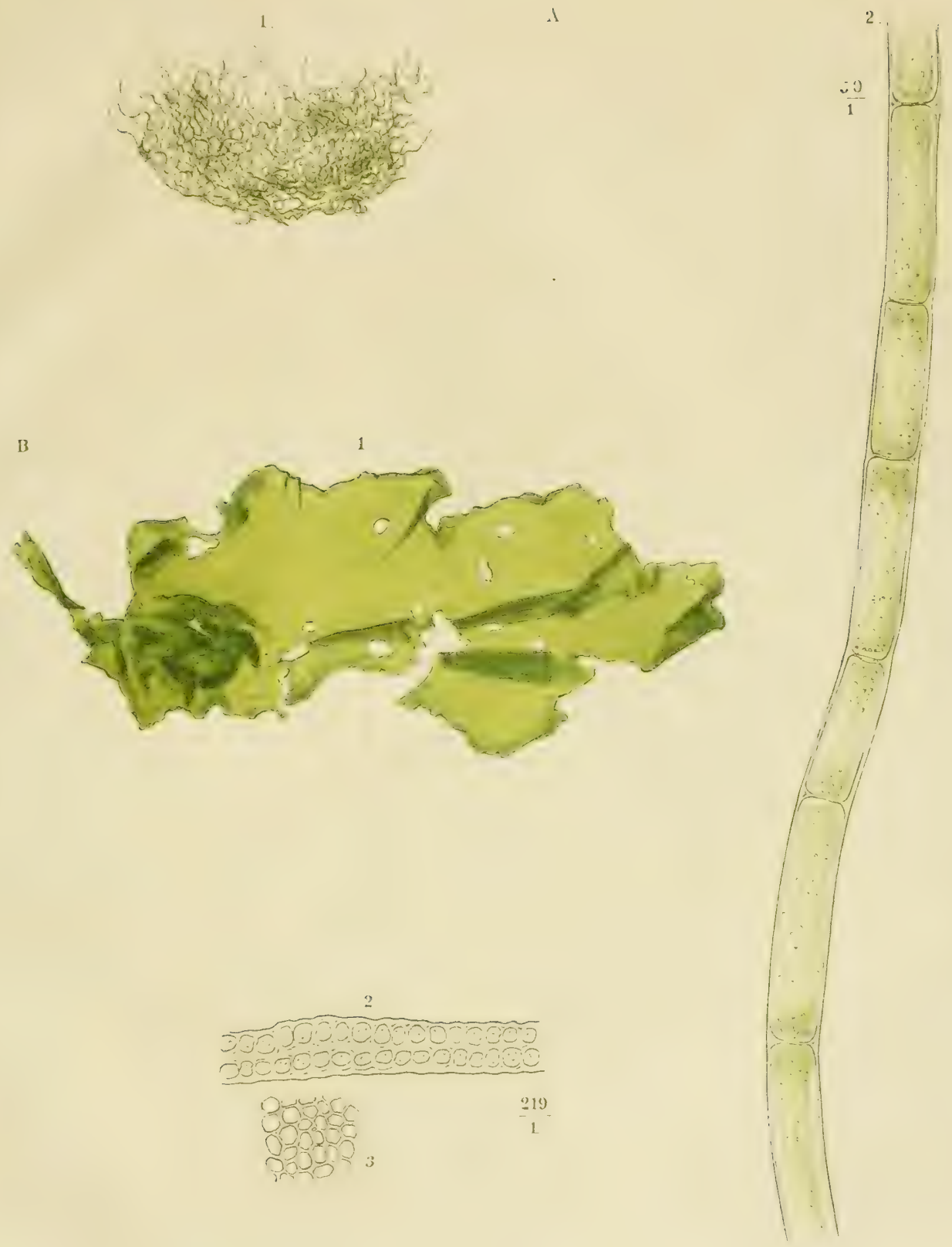



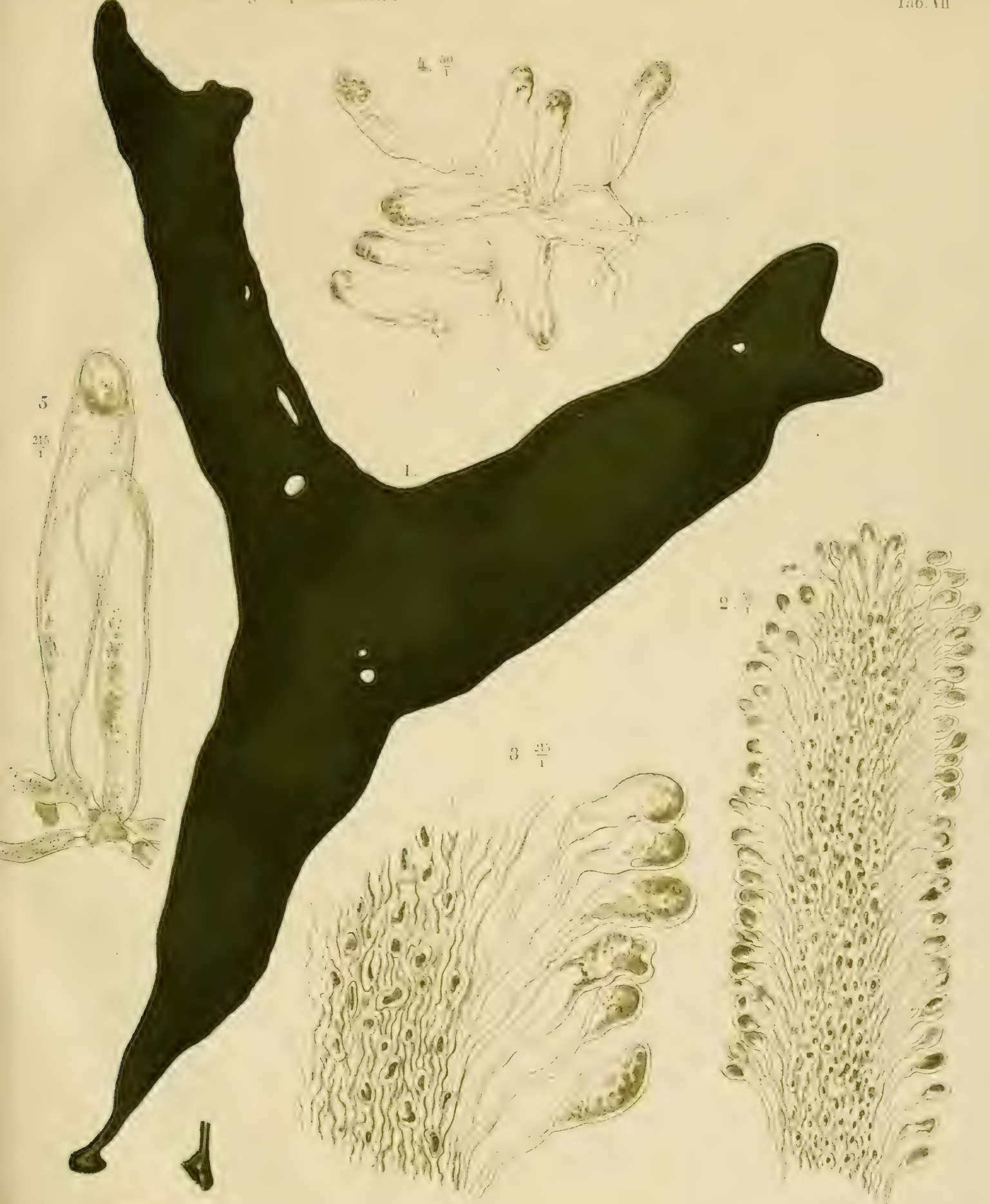


. 


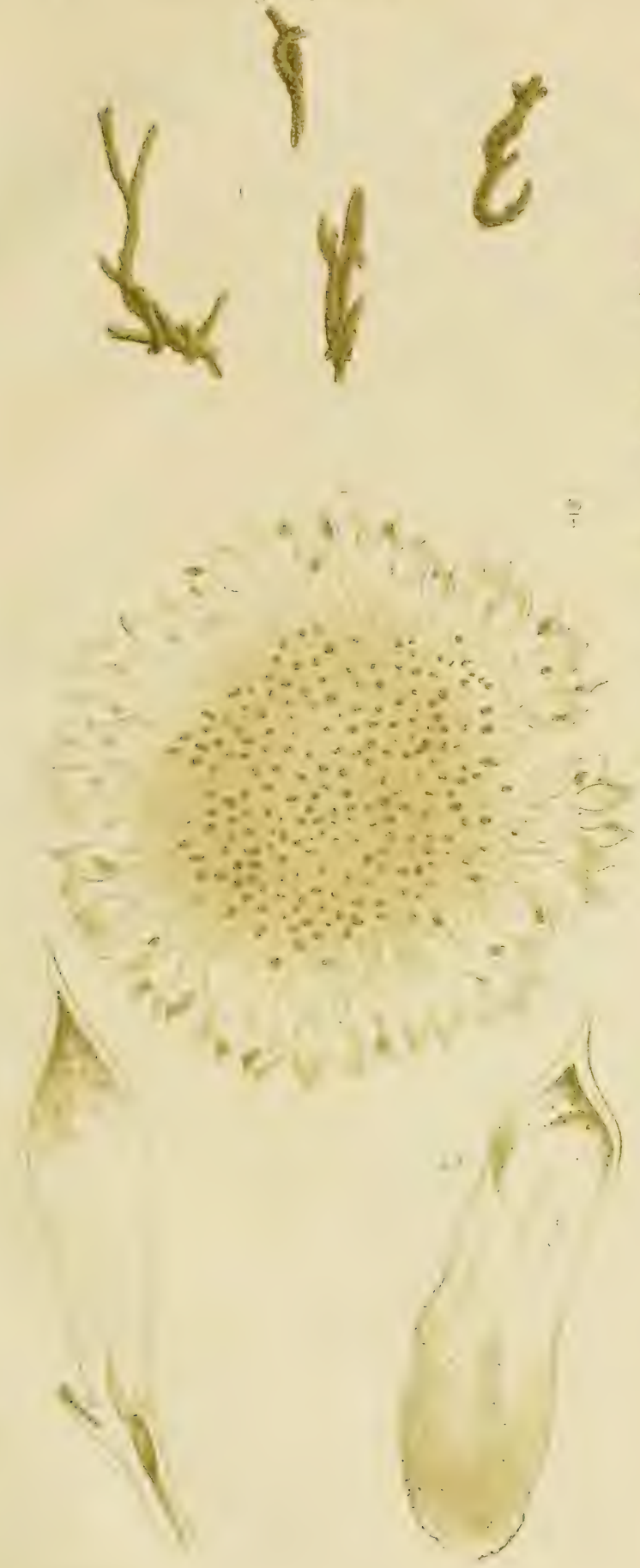

T... : ! 1!1
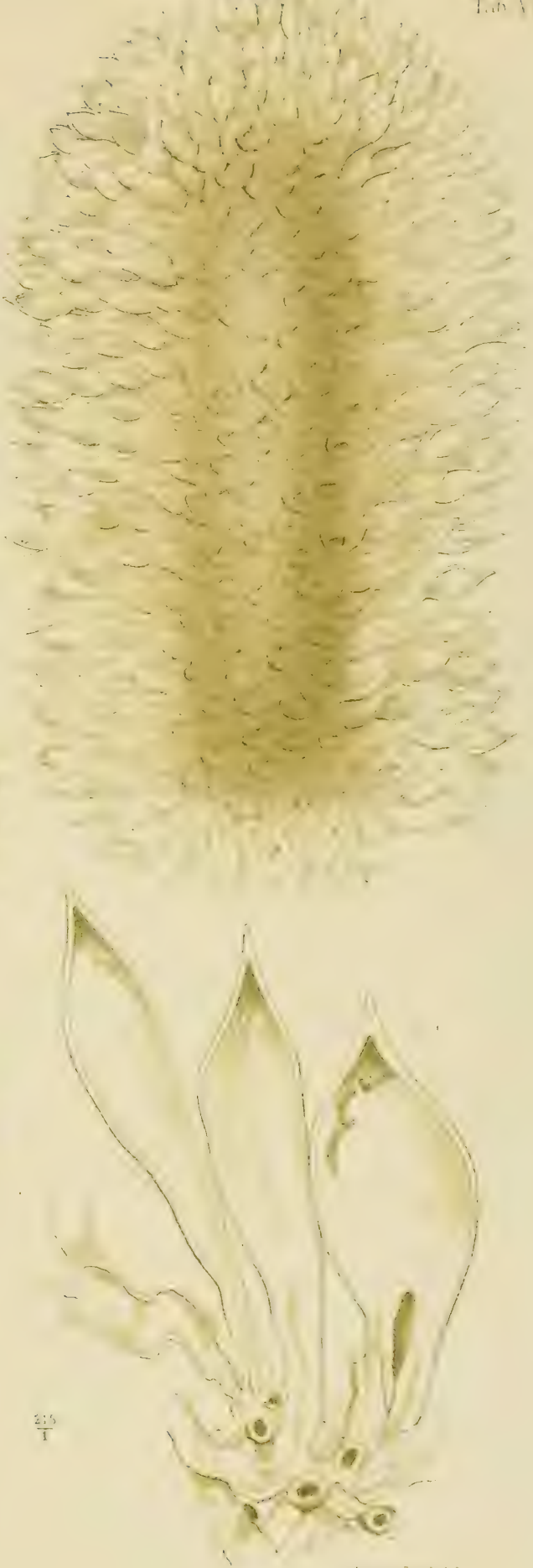


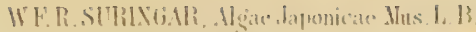

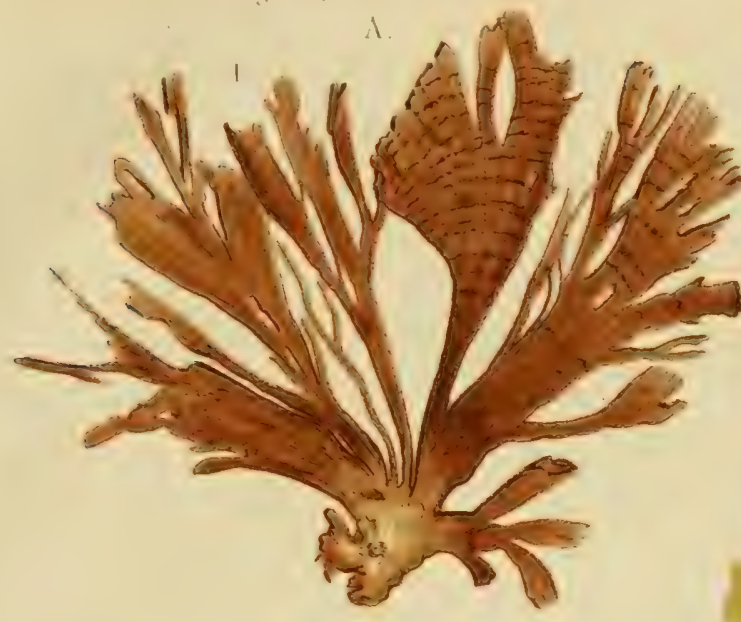

Li.

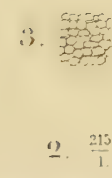

aconas
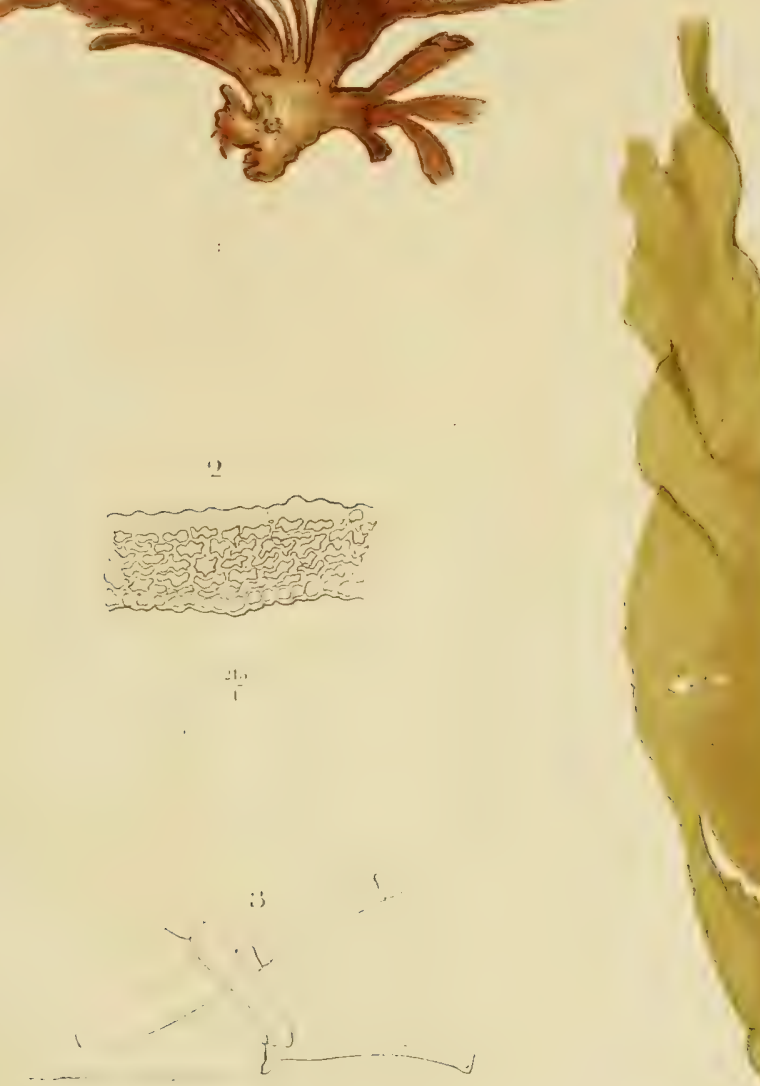
W.F.R.SLRINGAR, Algae Japonicae Mus. L. B

Tabe $\mathrm{X}$
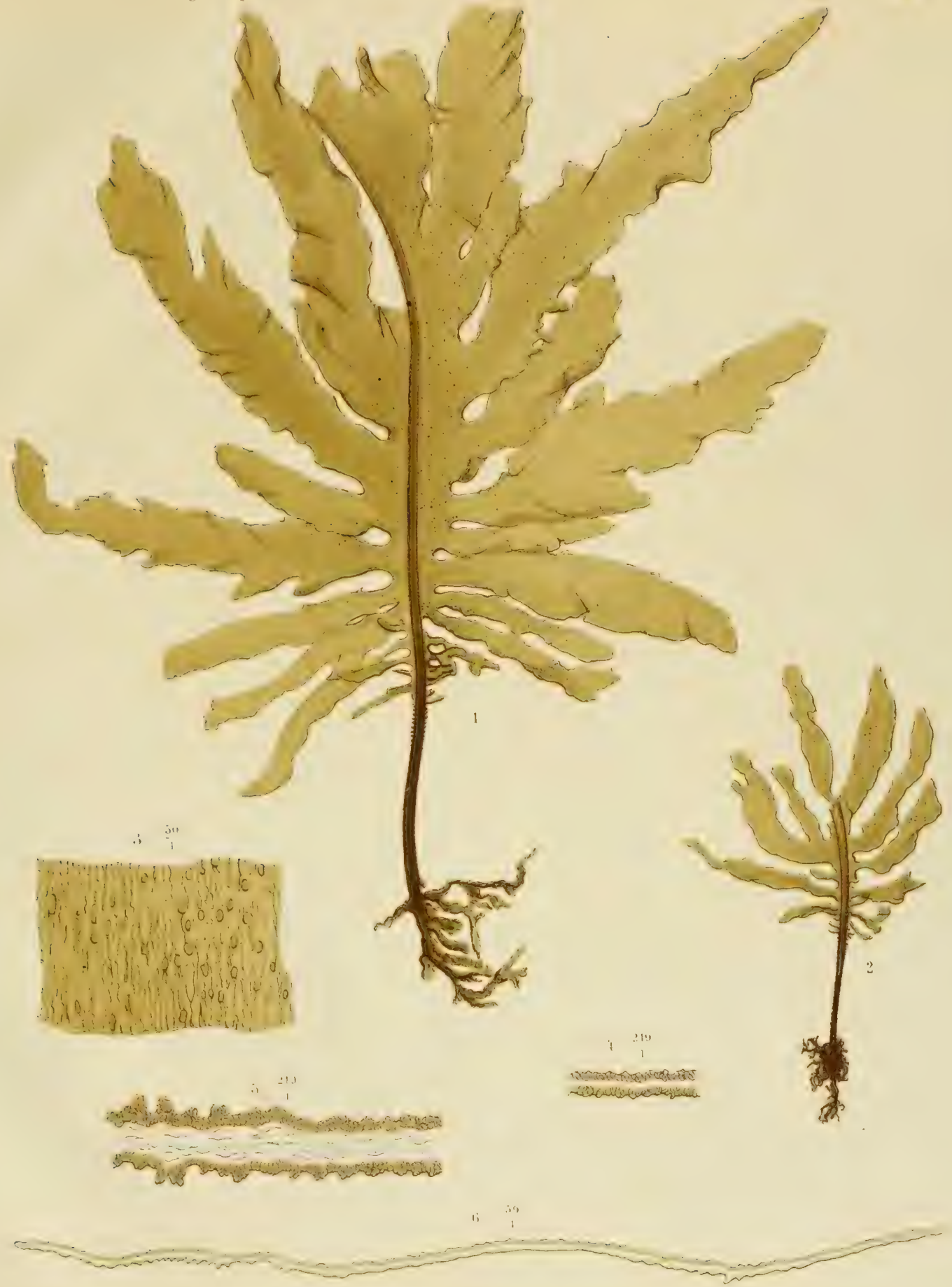

W. F. R.SURINGAR, Aggae Japonicae Mus. L.B.

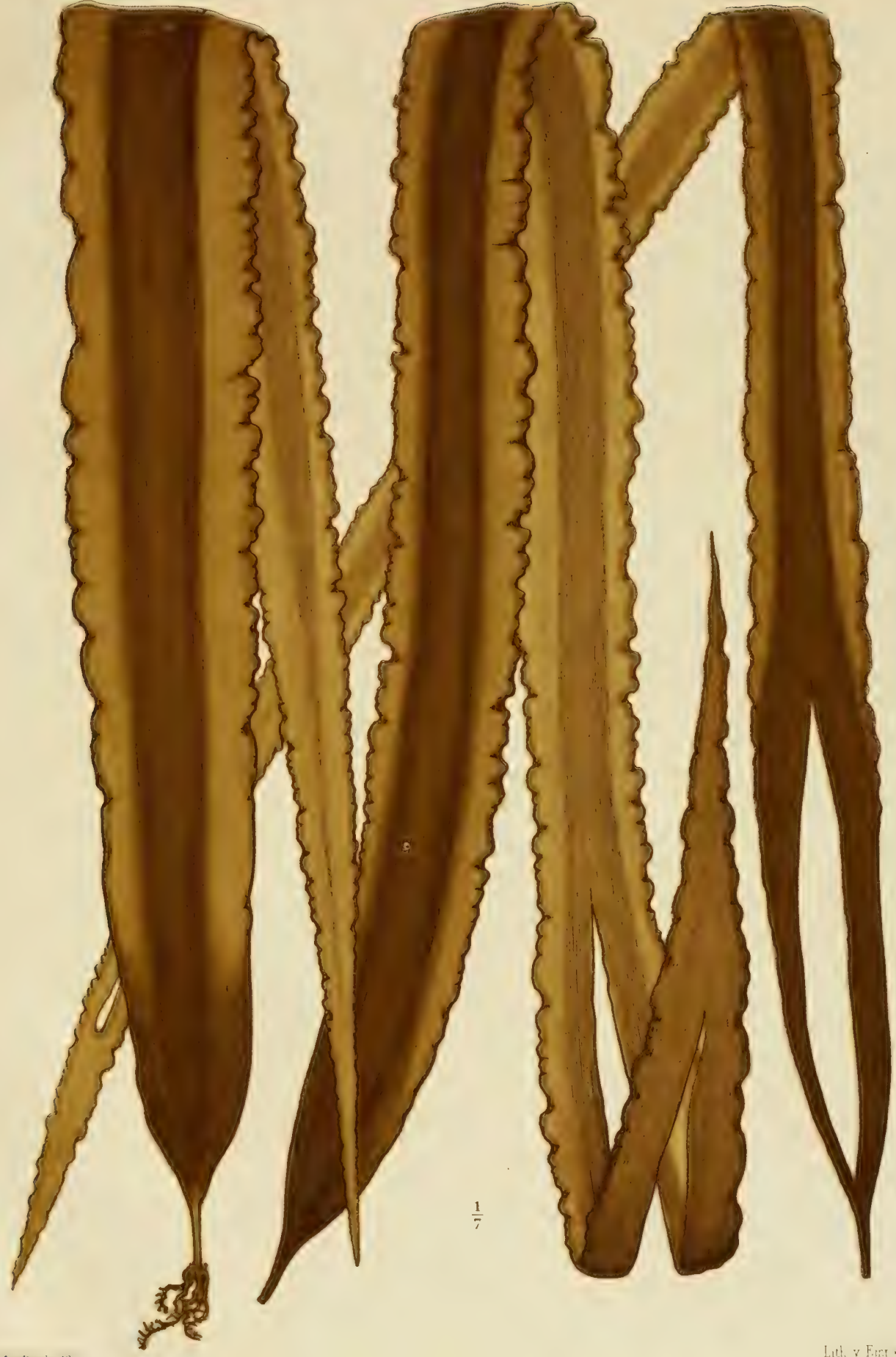


$$
\text { - }
$$ 
IF.R. SLRINGAR, Algae Japonicae Mus. L.B.

Tab. XII.

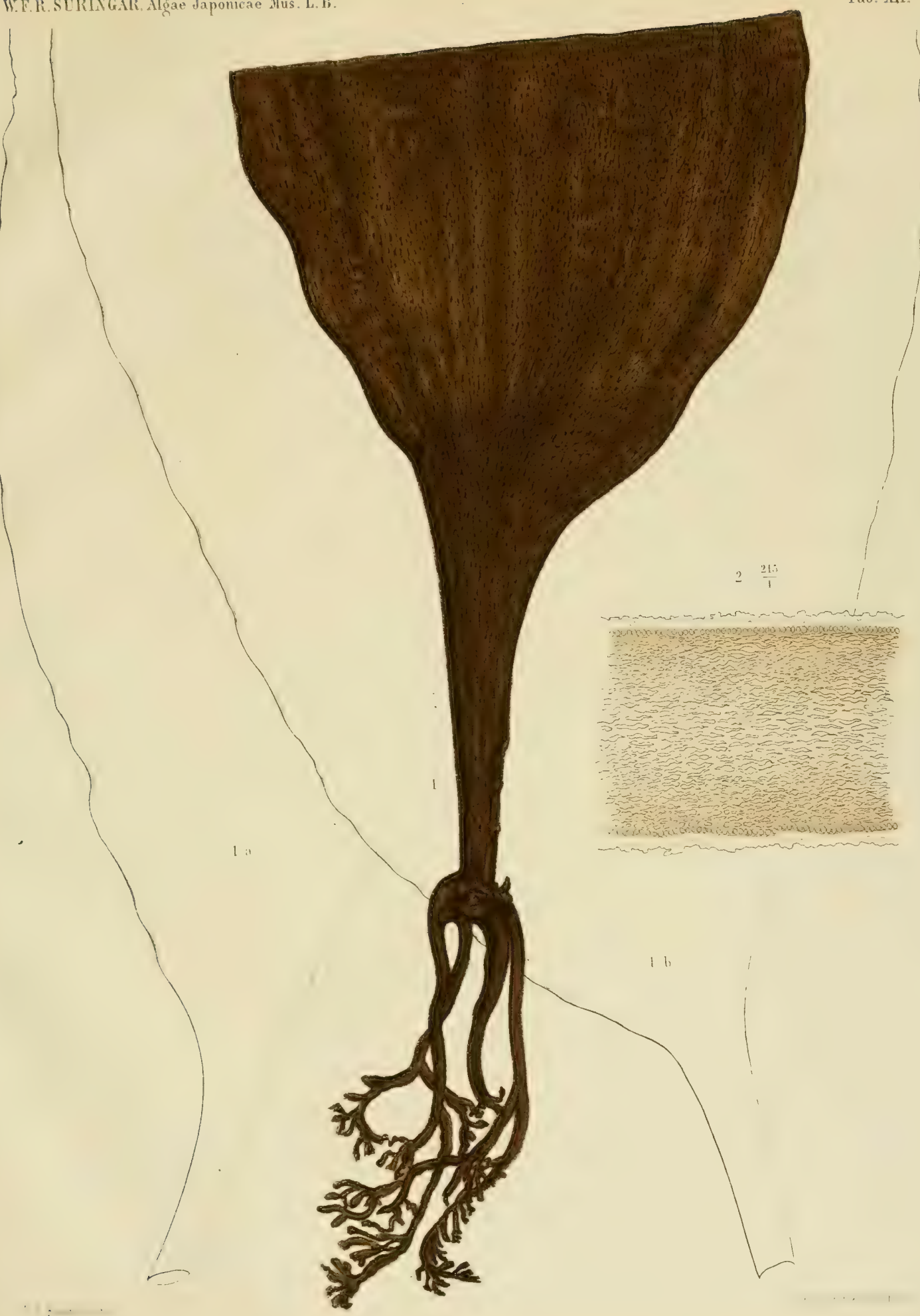


. 


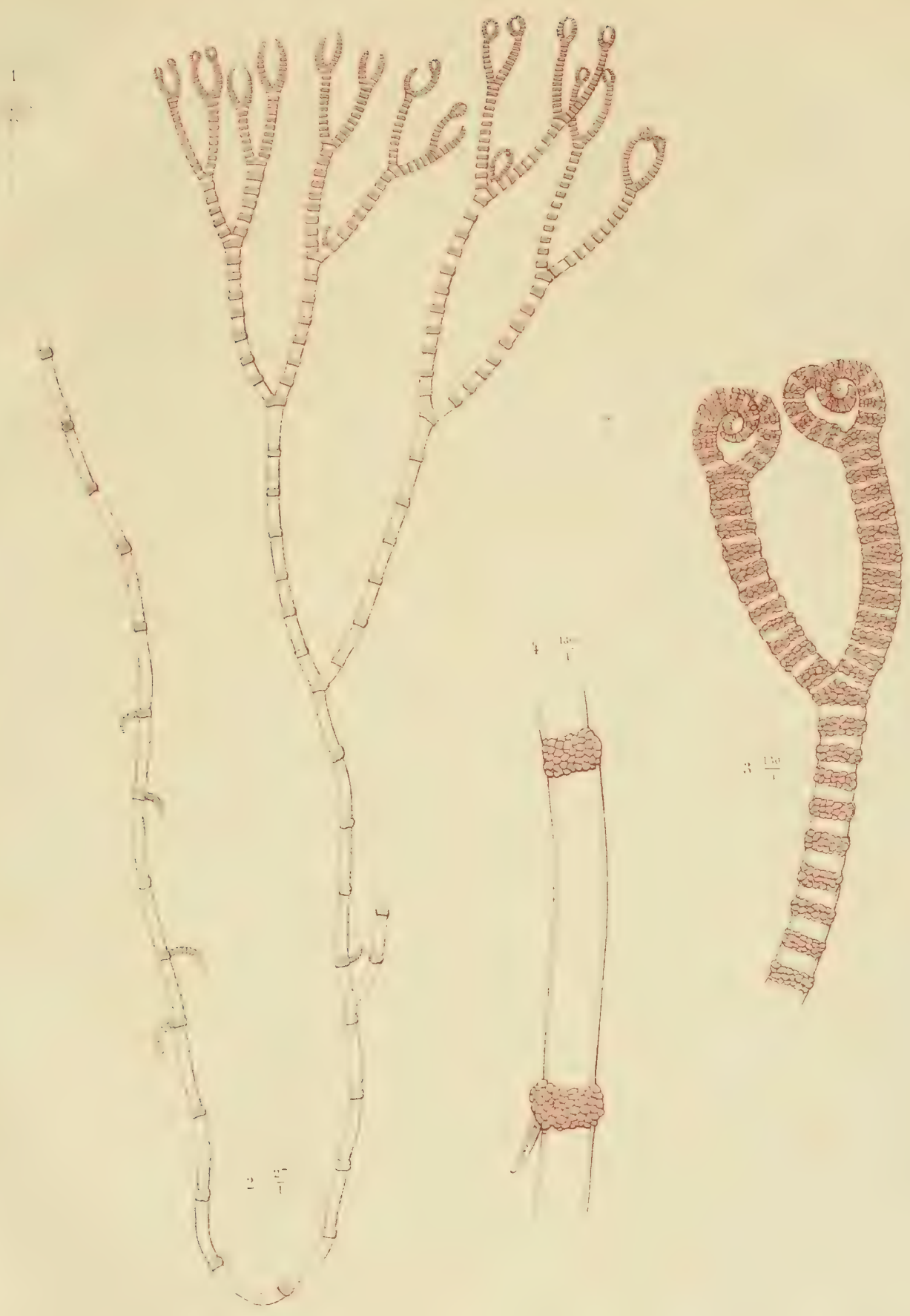




\section{.}


IV.F.R. StRIXG.JR, Algae Japonicne Jus L.B.
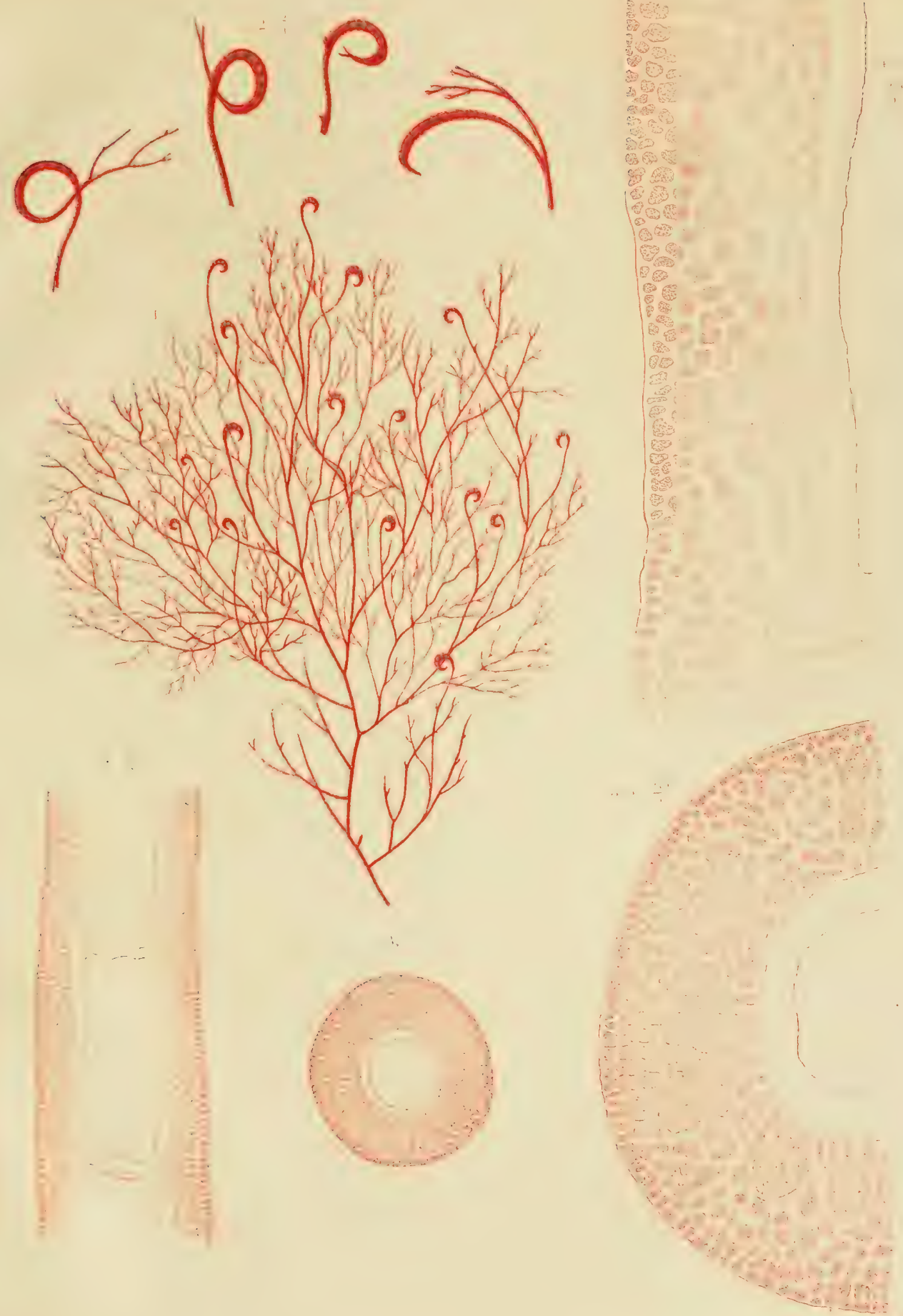
冫. 


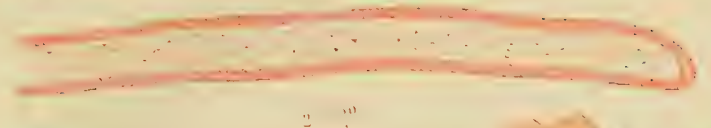

$\therefore \quad \frac{11}{1}$
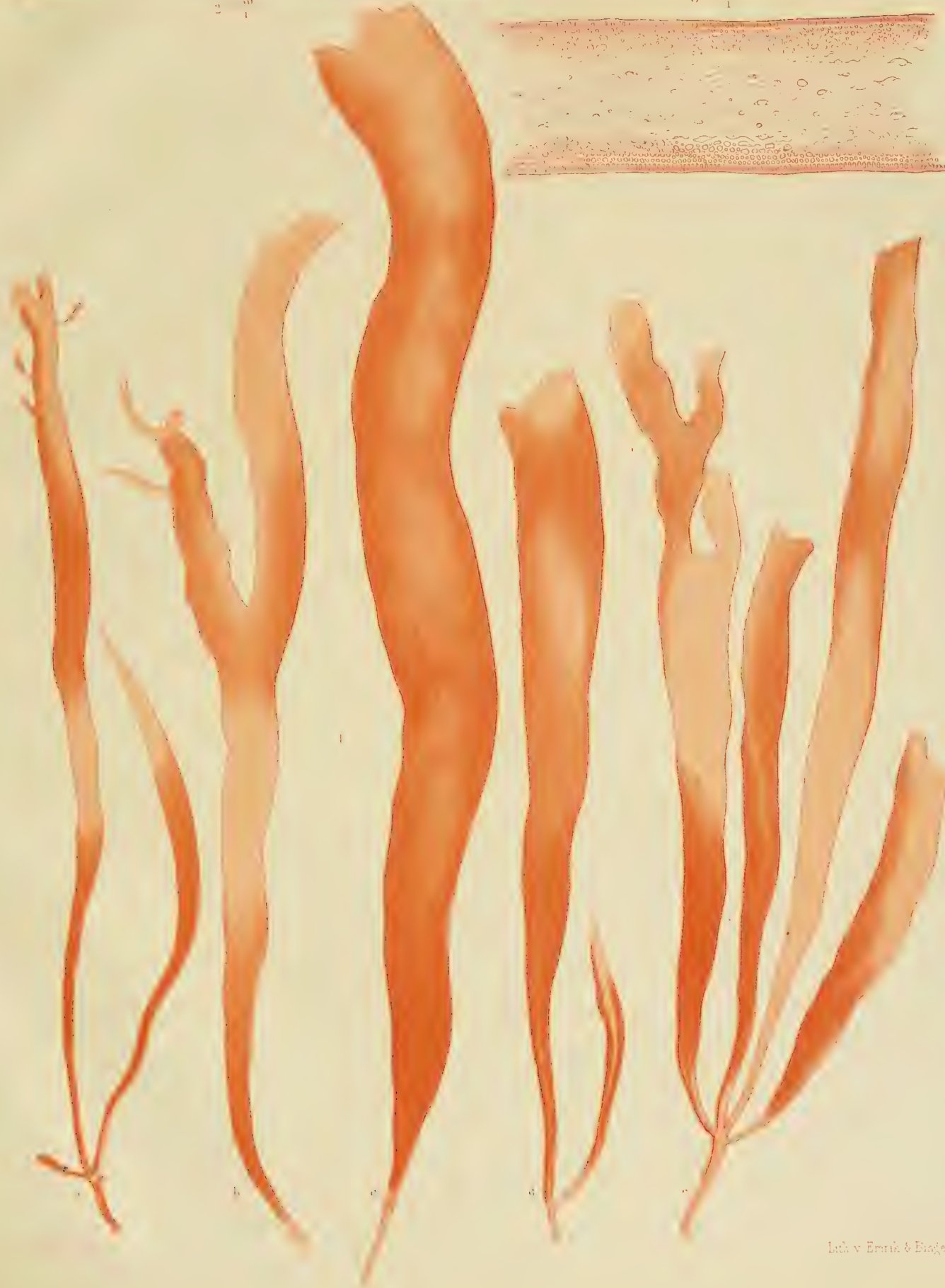

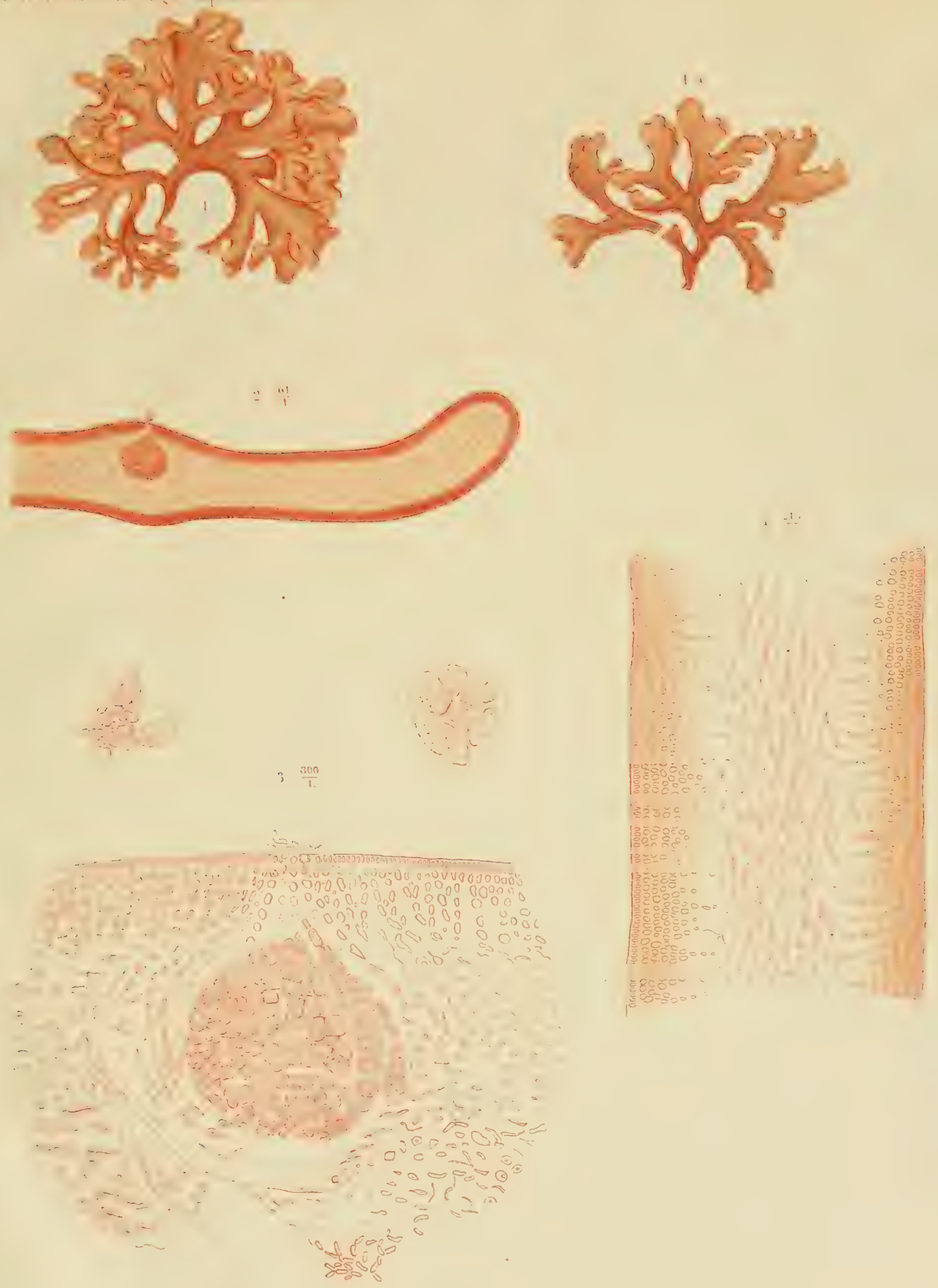

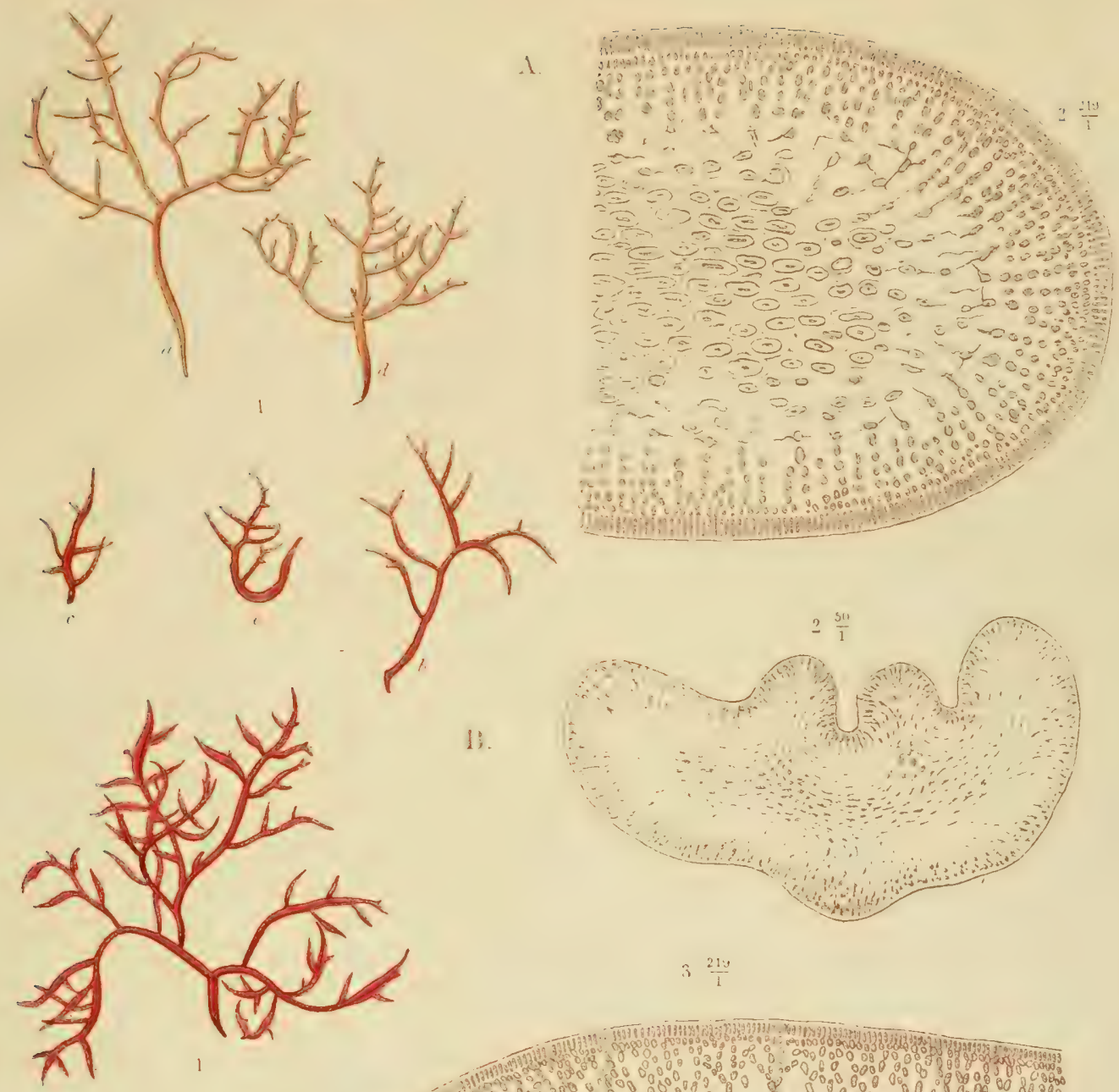

$3 \frac{214}{1}$

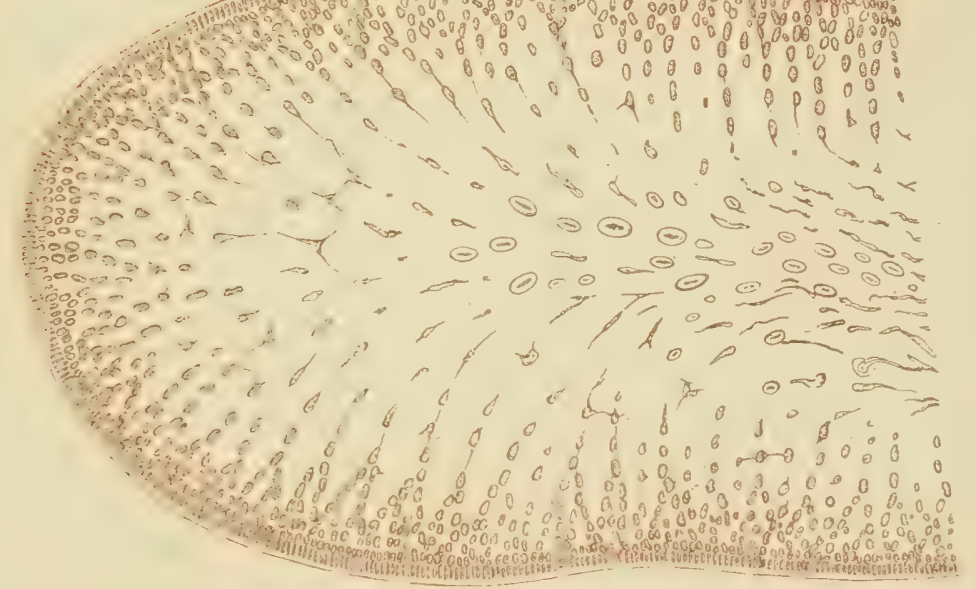




\section{.}




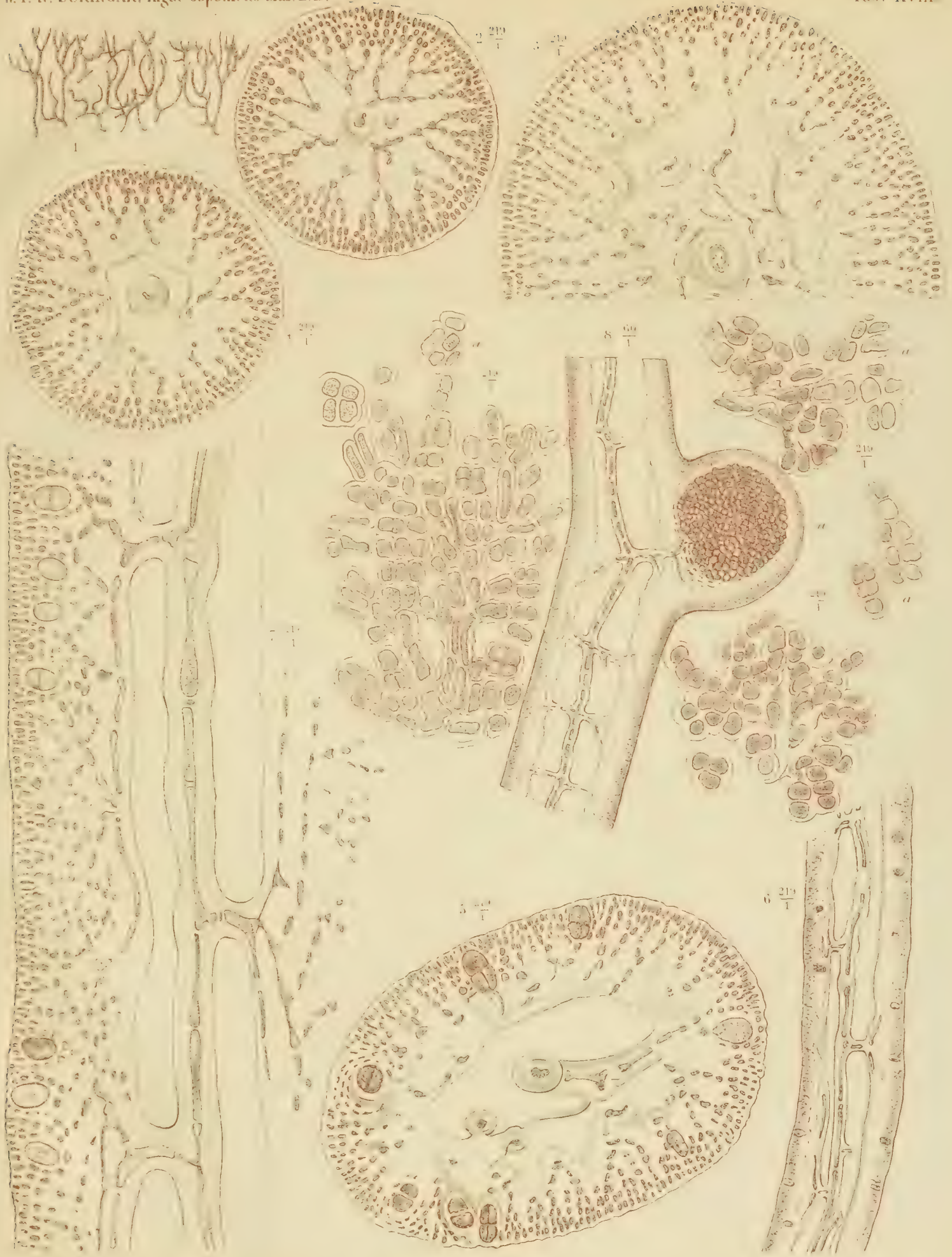




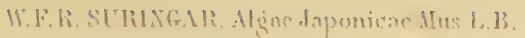
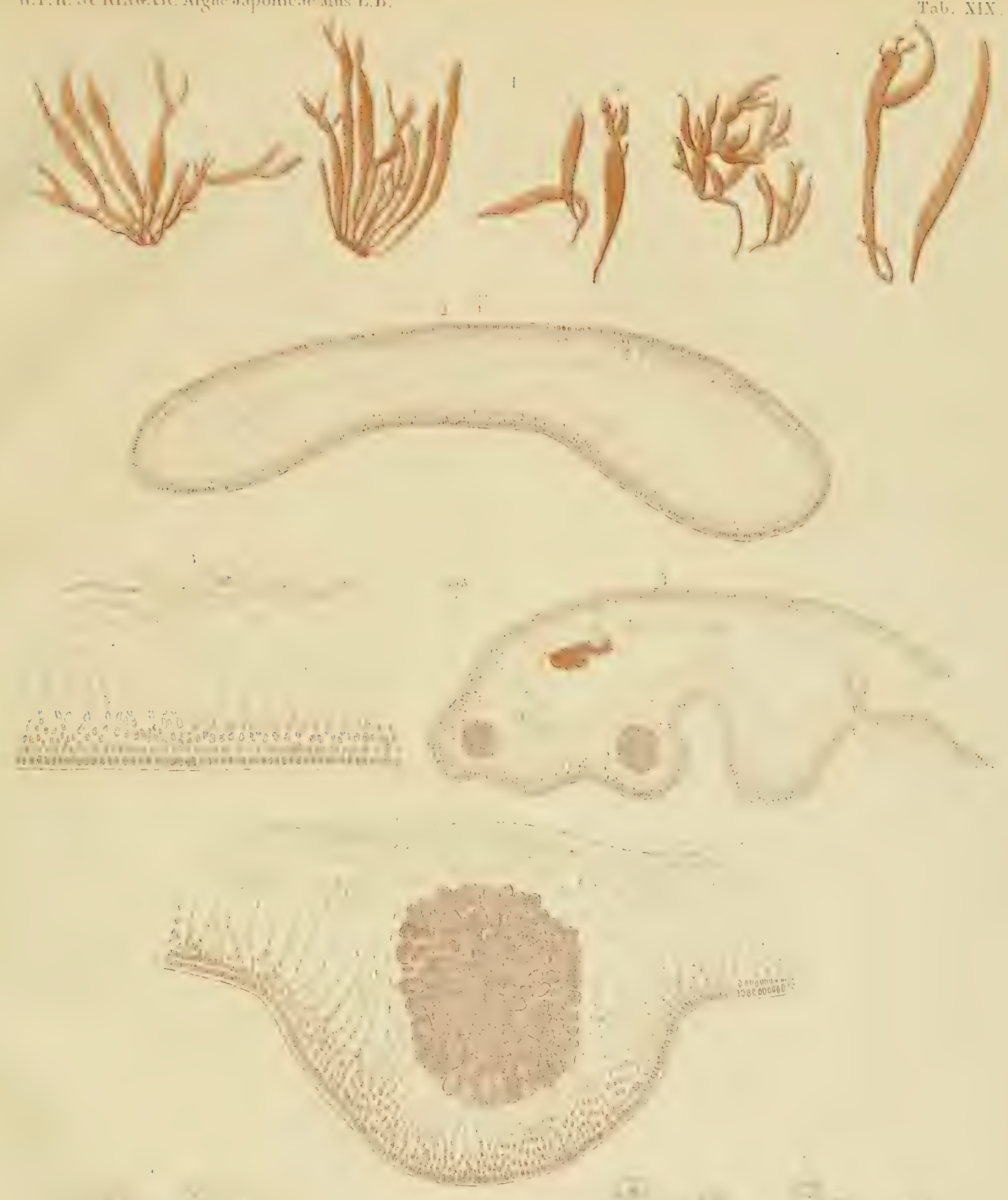



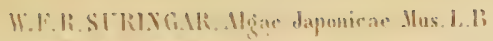
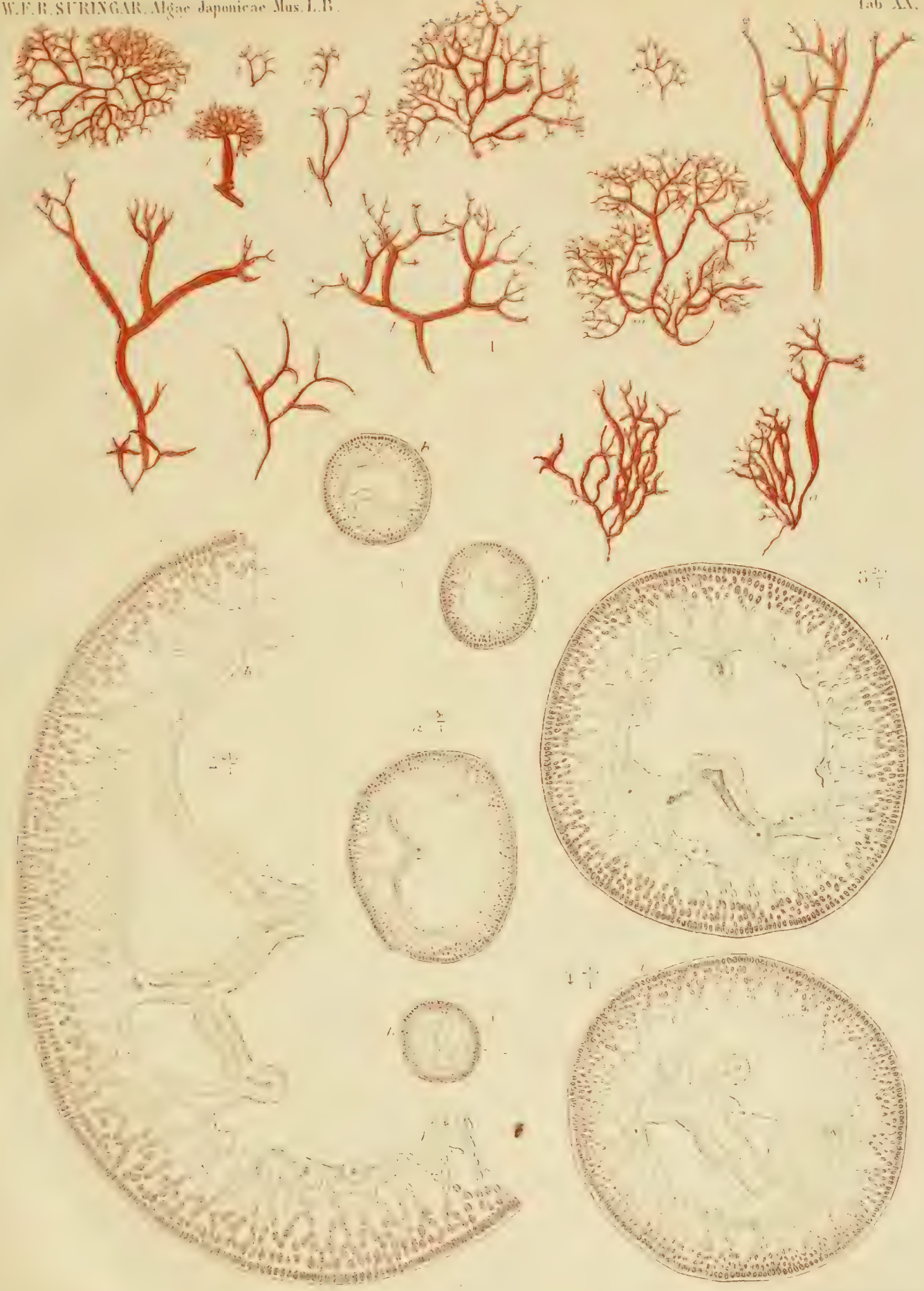

WF K.SIRIXG.MR, Algae Japonicae Mus L.B.

Tat. . IYL
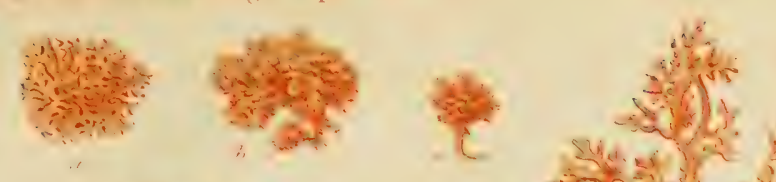

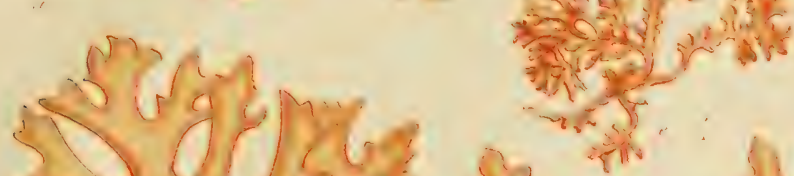

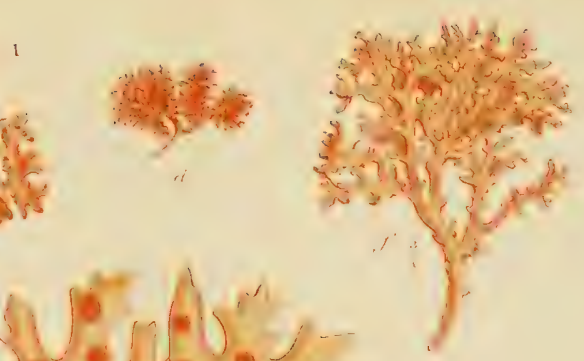

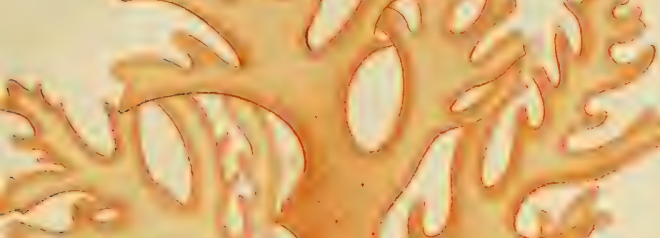

5
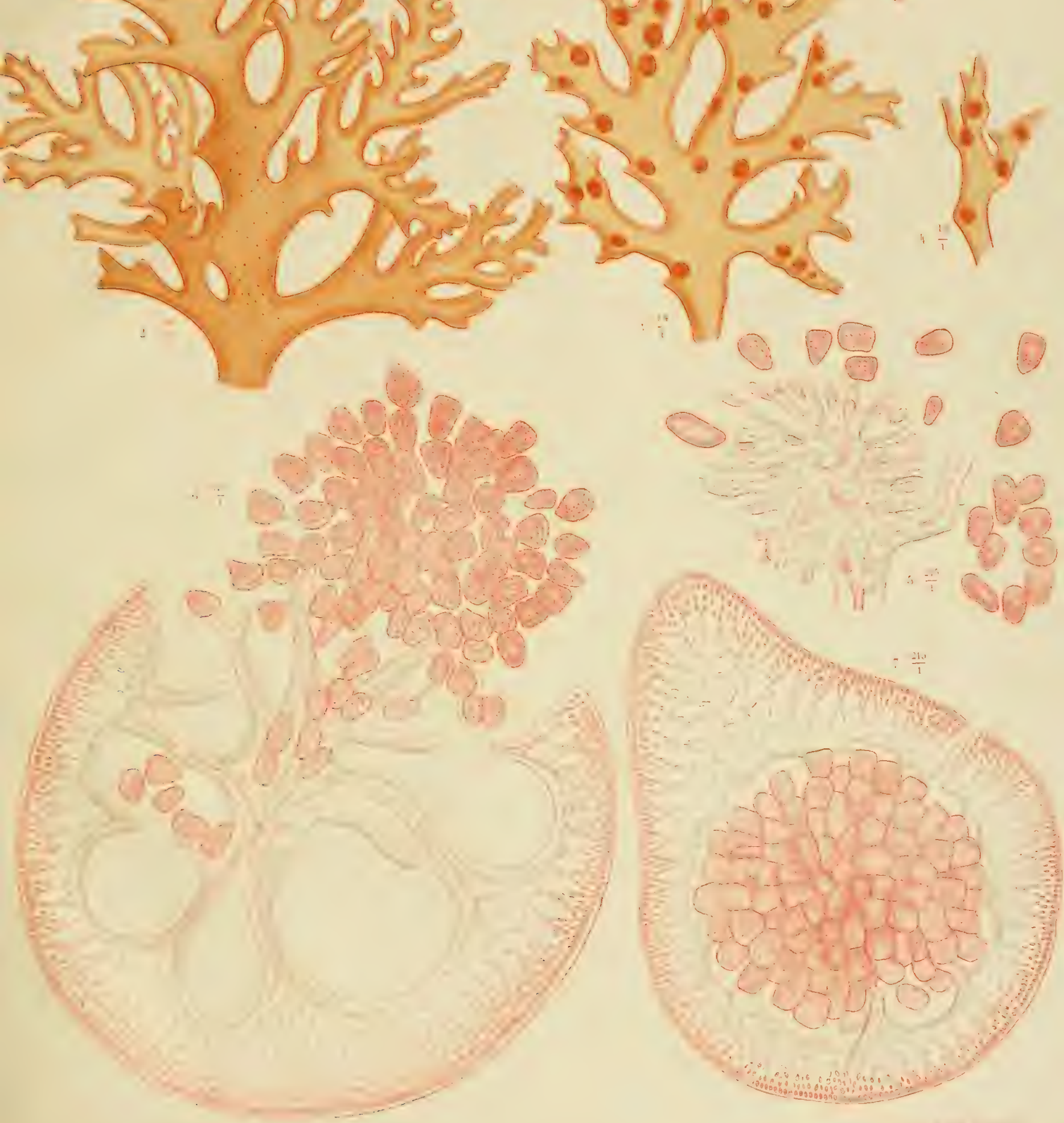


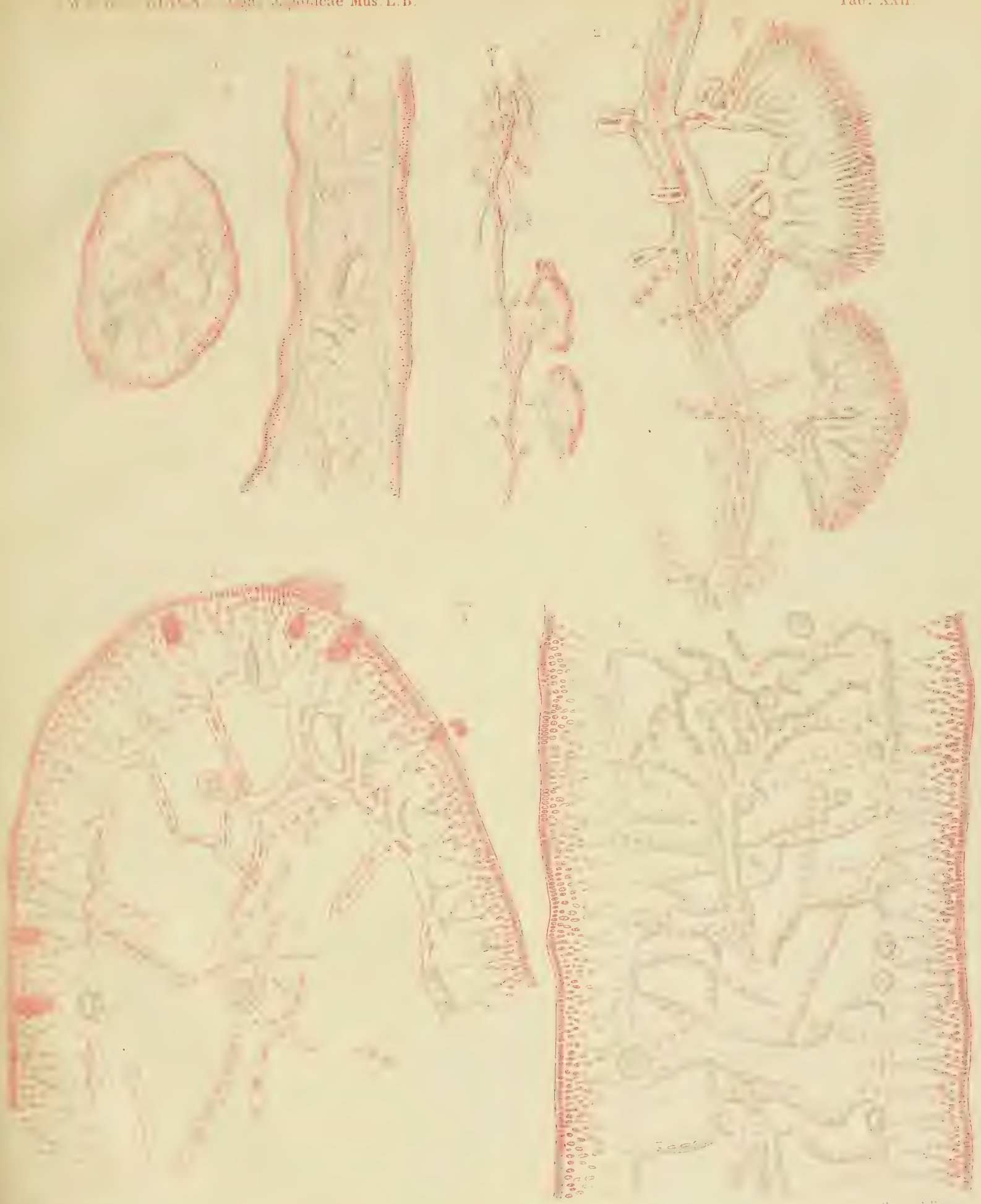



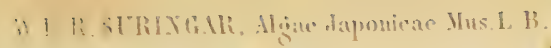
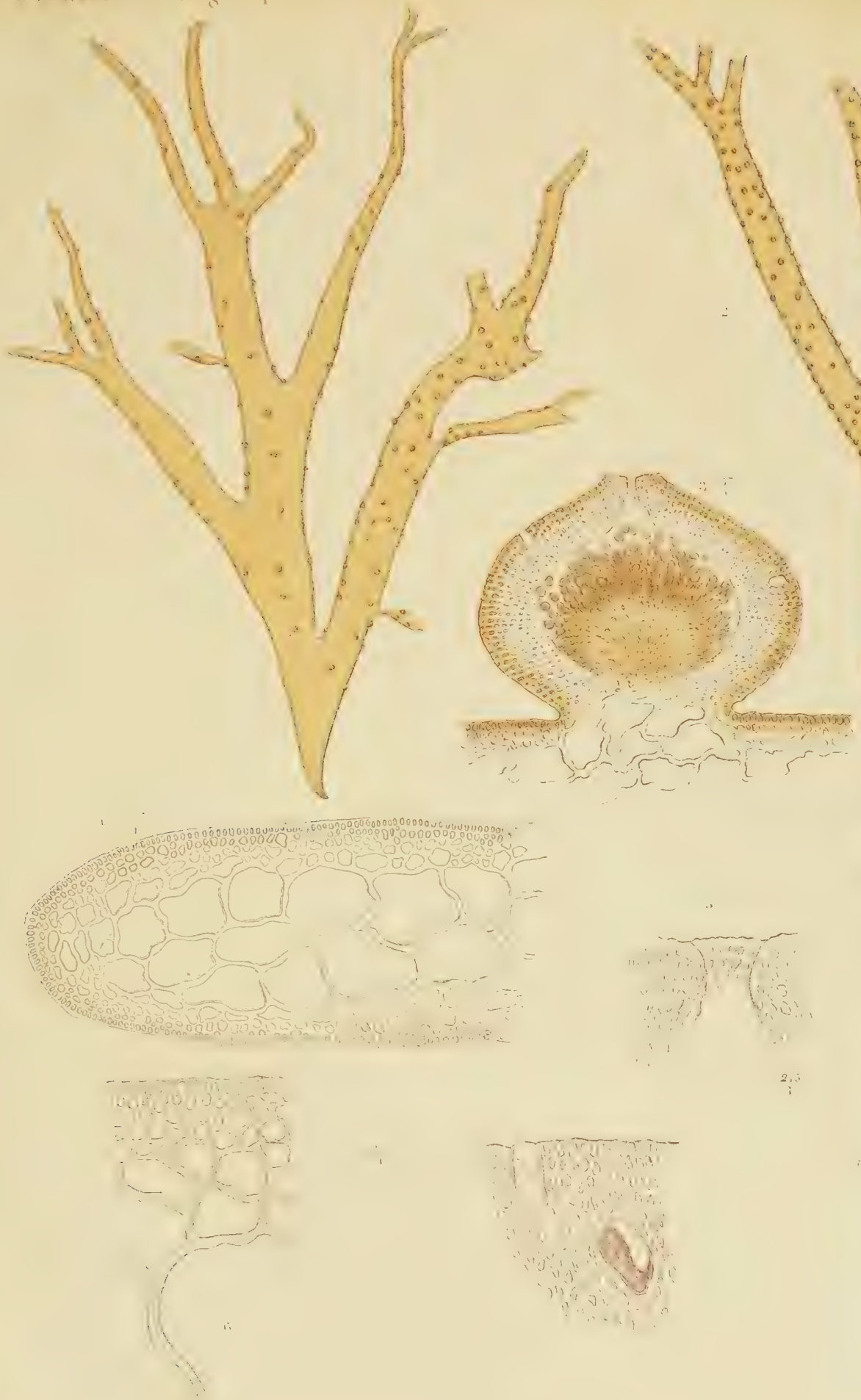
. 

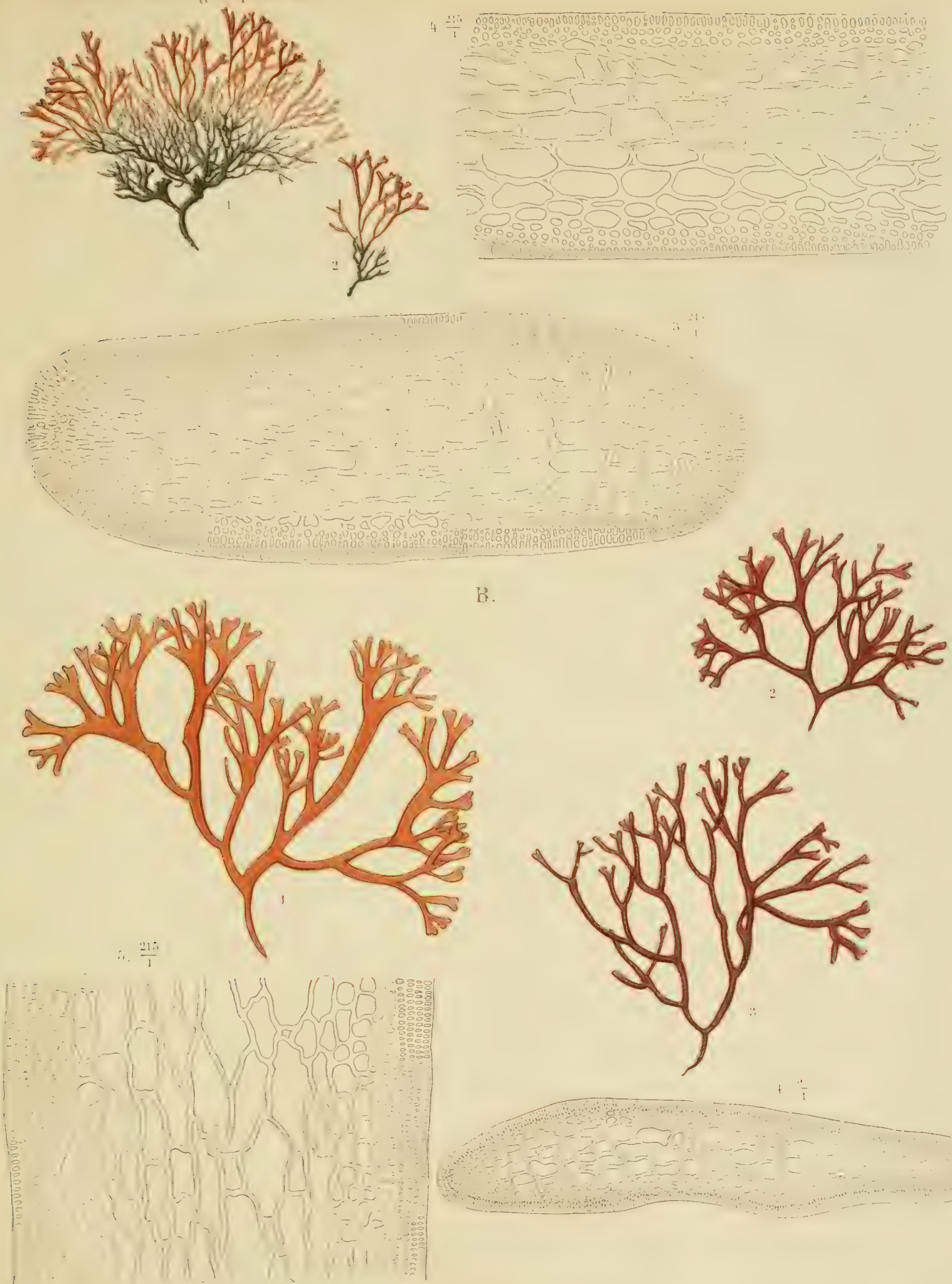

W. F. SURIXGAR, Alsae Japonica Mus.L. B.

Tab. XXV

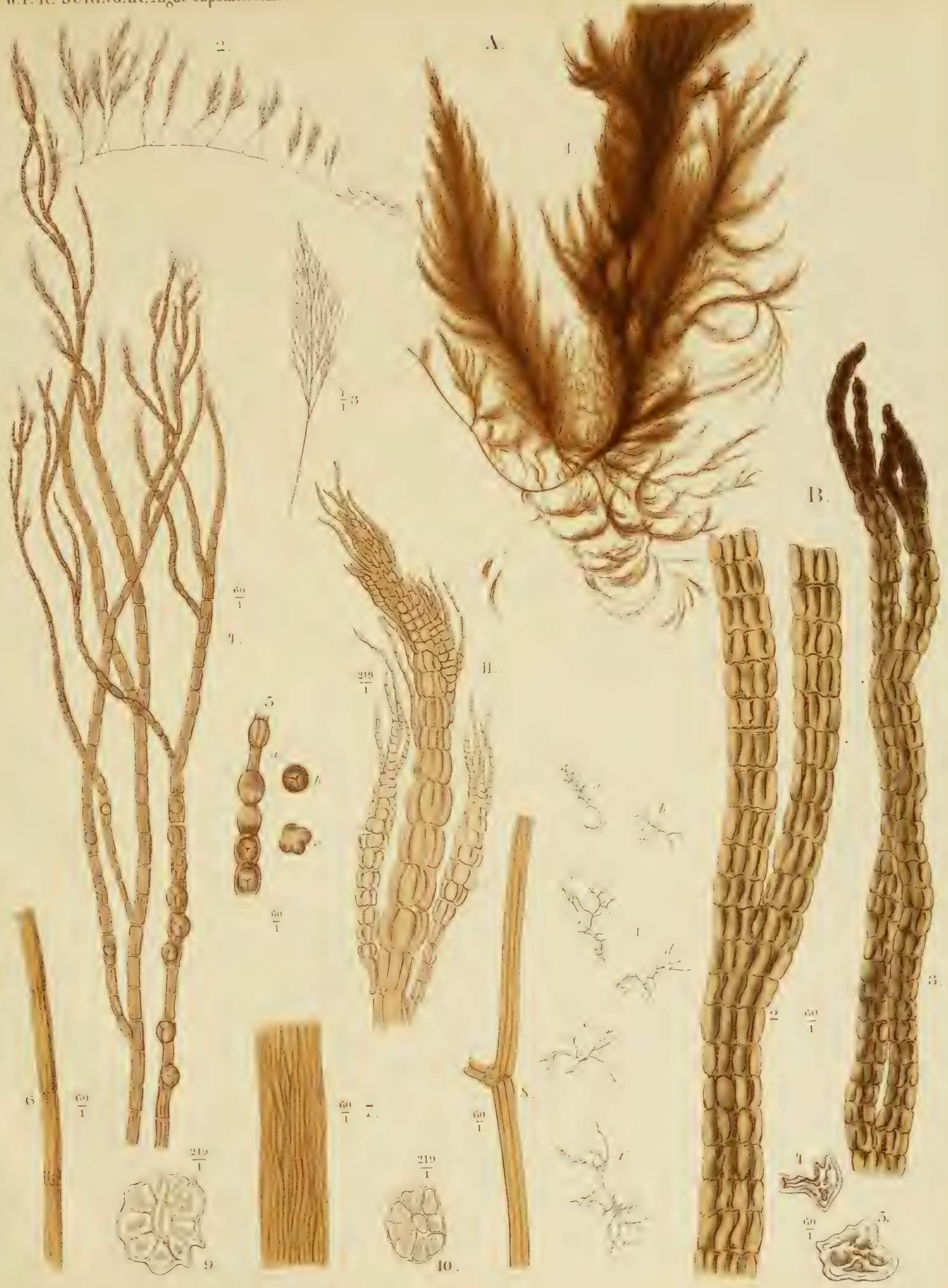






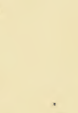


